Correspondence and proofs to:

Dr Simon Aldridge

Cardiff University

Address as below

AldridgeS@ cardiff.ac.uk

Tel: (029) 20875495

Fax: (029) 20874030

\title{
Halide abstraction as a route to cationic transition metal complexes containing two-coordinate gallium and indium ligand systems
}

Natalie R. Bunn, Simon Aldridge, ${ }^{*}$ Deborah L. Kays (née Coombs), Natalie D. Coombs, Andrea Rossin, David J. Willock, Joanne K. Day Cameron Jones and Li-ling Ooi.

Centre for Fundamental and Applied Main Group Chemistry, School of Chemistry, Cardiff University, Main Building, Park Place, Cardiff, UK CF10 3AT. www.cf.ac.uk/chemy/cfamgc 


\section{Contents}

1. Details of crystal structure of compound 7

$\begin{array}{lll}2 . & \text { Details of crystal structure of compound } \mathbf{8} & 15\end{array}$

3. Details of crystal structure of compound $\mathbf{1 0} 27$

4. Details of crystal structure of compound $\mathbf{1 4} \quad 44$

5. $\mathrm{X}, \mathrm{y}, \mathrm{z}$ coordinates for DFT optimised geometry of $\left[\left\{\mathrm{Cp} * \mathrm{Fe}(\mathrm{CO})_{2}\right\}(\mu-\mathrm{Ga})\right]^{+}$

6. $\mathrm{X}, \mathrm{y}, \mathrm{z}$ coordinates for DFT optimised geometry of $\left[\left\{\mathrm{Cp} * \mathrm{Fe}(\mathrm{CO})_{2}\right\}(\mu-\mathrm{In})\right]^{+}$and $\begin{array}{ll}\text { rotational energy profile } & 61\end{array}$

7. $\quad{ }^{1} \mathrm{H}$ NMR spectra for new compounds $\quad 64$ 


\section{Details of the crystal structure of compound 7}

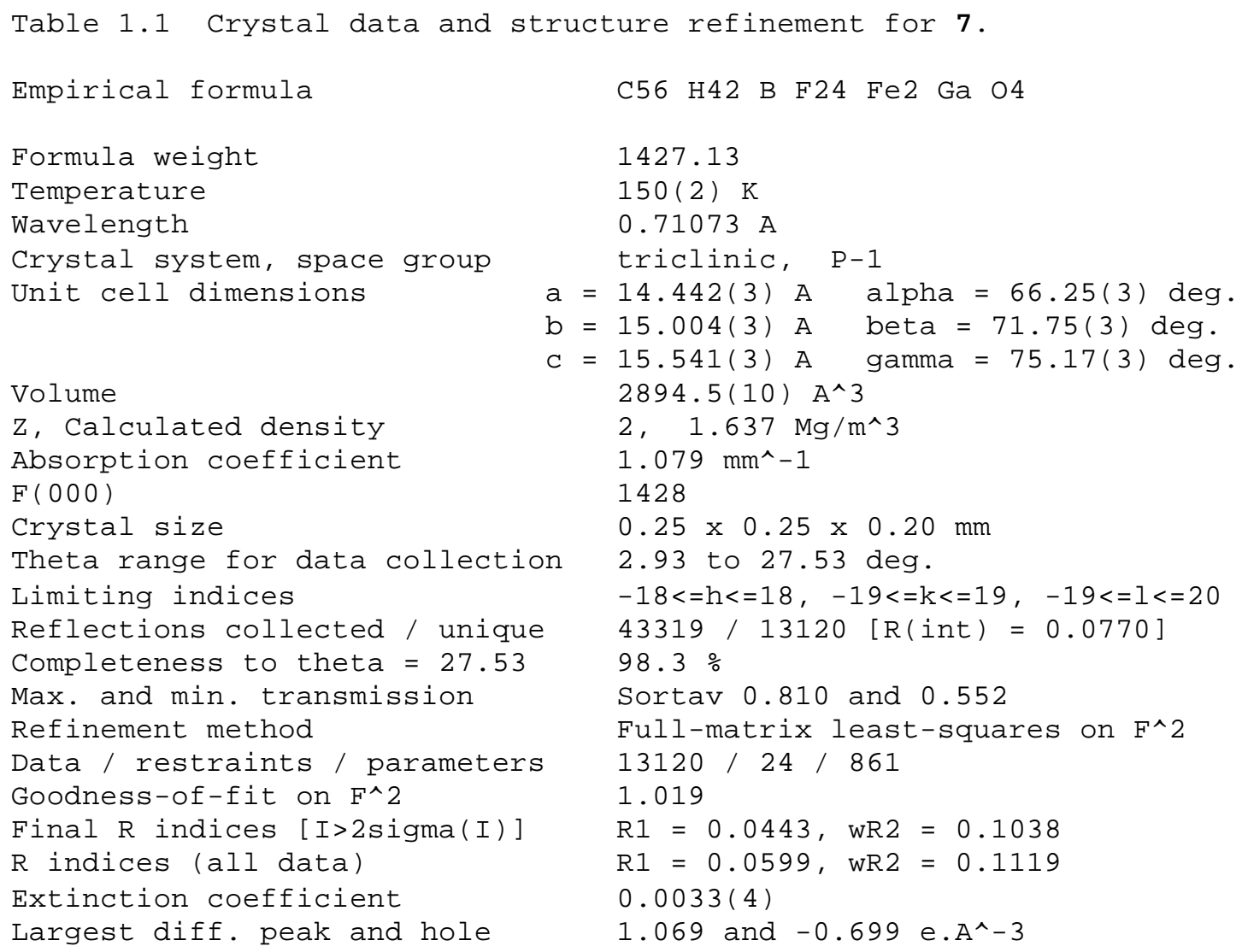


Table 1.2 Atomic coordinates ( $x$ 10^4) and equivalent isotropic displacement parameters $\left(A^{\wedge} 2 \times 10^{\wedge} 3\right)$ for 7 .

$\mathrm{U}(\mathrm{eq})$ is defined as one third of the trace of the orthogonalized Uij tensor.

\begin{tabular}{|c|c|c|c|c|}
\hline & $\mathrm{x}$ & y & $\mathrm{z}$ & $\mathrm{U}(\mathrm{eq})$ \\
\hline $\mathrm{Ga}(1)$ & $8494(1)$ & $8937(1)$ & $4959(1)$ & $24(1)$ \\
\hline $\operatorname{Fe}(1)$ & $9280(1)$ & $7950(1)$ & $6152(1)$ & $22(1)$ \\
\hline $\mathrm{Fe}(2)$ & 7727 (1) & $9905(1)$ & $3753(1)$ & $22(1)$ \\
\hline$F(1)$ & $4847(17)$ & 3265 (15) & $3690(15)$ & $43(3)$ \\
\hline$F(1 A)$ & $4760(30)$ & $3130(30)$ & $3890(30)$ & $49(6)$ \\
\hline$F(2)$ & $6271(3)$ & $2457(4)$ & $3776(9)$ & $61(3)$ \\
\hline$F(2 A)$ & $6243(6)$ & $2685(7)$ & $3138(14)$ & $60(4)$ \\
\hline$F(3)$ & $5279(7)$ & $2654(7)$ & $5057(4)$ & $60(2)$ \\
\hline$F(3 A)$ & $5848(14)$ & $2310(7)$ & $4671(12)$ & $73(6)$ \\
\hline$F(4)$ & $7865(9)$ & $4302(9)$ & $5250(9)$ & $53(3)$ \\
\hline$F(4 A)$ & $7729(10)$ & $4230(7)$ & $5361(8)$ & $72(4)$ \\
\hline$F(5)$ & $6490(8)$ & $4988(14)$ & $5817(6)$ & $71(4)$ \\
\hline$F(5 A)$ & $6681(9)$ & $5474(13)$ & $5497(14)$ & $86(4)$ \\
\hline$F(6)$ & $7494(17)$ & $5871(7)$ & $4678(10)$ & $69(4)$ \\
\hline$F(6 A)$ & $7989(10)$ & $5649(9)$ & $4395(7)$ & $64(3)$ \\
\hline$F(7)$ & $2942(2)$ & $8581(2)$ & $4260(2)$ & $63(1)$ \\
\hline$F(8)$ & $3928(2)$ & $9452(2)$ & $3046(2)$ & $64(1)$ \\
\hline$F(9)$ & $2432(2)$ & $9555(2)$ & $3021(2)$ & $56(1)$ \\
\hline$F(10)$ & $1377(2)$ & $6805(2)$ & $2783(2)$ & $68(1)$ \\
\hline$F(11)$ & $2213(2)$ & $6971(2)$ & $1338(2)$ & $82(1)$ \\
\hline $\mathrm{F}(12)$ & $2454(2)$ & $5640(2)$ & $2492(2)$ & $52(1)$ \\
\hline$F(13)$ & $4187(1)$ & $6567(2)$ & $-690(2)$ & $60(1)$ \\
\hline$F(14)$ & $5216(2)$ & $5771(2)$ & $-1553(2)$ & $60(1)$ \\
\hline$F(15)$ & $5268(2)$ & $7285(1)$ & $-1901(1)$ & $49(1)$ \\
\hline$F(16)$ & $8629(2)$ & $4647(2)$ & $-1151(2)$ & $68(1)$ \\
\hline$F(17)$ & $8201(2)$ & $3700(2)$ & $278(2)$ & $102(1)$ \\
\hline$F(18)$ & $9009(2)$ & $4849(2)$ & $-65(3)$ & $91(1)$ \\
\hline$F(19)$ & $6341(3)$ & $10288(2)$ & $-848(2)$ & $116(1)$ \\
\hline$F(20)$ & $5742(3)$ & $10562(2)$ & $392(2)$ & $102(1)$ \\
\hline$F(21)$ & $7188(3)$ & $10836(2)$ & $-327(4)$ & $159(2)$ \\
\hline$F(22)$ & $9920(2)$ & $7523(2)$ & $127(2)$ & $76(1)$ \\
\hline$F(23)$ & $9585(2)$ & $6786(2)$ & $1663(2)$ & $57(1)$ \\
\hline$F(24)$ & $9775(2)$ & $8297(2)$ & $1063(2)$ & $63(1)$ \\
\hline$O(1)$ & $9630(2)$ & $9763(2)$ & $6185(2)$ & $35(1)$ \\
\hline$O(2)$ & $11074(2)$ & $7731(2)$ & $4698(2)$ & $54(1)$ \\
\hline$O(3)$ & $8378(2)$ & $11659(2)$ & $3601(2)$ & $42(1)$ \\
\hline$O(4)$ & $5900(2)$ & $10065(2)$ & $5202(2)$ & $58(1)$ \\
\hline$C(1)$ & $9475(2)$ & $9054(2)$ & $6174(2)$ & $25(1)$ \\
\hline$C(2)$ & $10363(2)$ & $7842(2)$ & $5254(2)$ & $33(1)$ \\
\hline$C(3)$ & $8129(2)$ & $10954(2)$ & $3691(2)$ & $29(1)$ \\
\hline$C(4)$ & $6623(2)$ & $10000(2)$ & $4642(2)$ & $35(1)$ \\
\hline$C(5)$ & $8158(2)$ & $7044(2)$ & $6765(2)$ & $26(1)$ \\
\hline$C(6)$ & $8145(2)$ & $7517(2)$ & $7409(2)$ & $28(1)$ \\
\hline$C(7)$ & $9082(2)$ & $7202(2)$ & $7661(2)$ & $31(1)$ \\
\hline$C(8)$ & $9673(2)$ & $6558(2)$ & $7174(2)$ & 31 (1) \\
\hline$C(9)$ & $9102(2)$ & $6466(2)$ & $6610(2)$ & $28(1)$ \\
\hline$C(10)$ & $7292(2)$ & $7055(2)$ & $6416(2)$ & $35(1)$ \\
\hline$C(11)$ & $7283(3)$ & $8134(2)$ & $7838(3)$ & $43(1)$ \\
\hline$C(12)$ & $9353(3)$ & $7449(3)$ & $8390(2)$ & $50(1)$ \\
\hline$C(13)$ & $10682(3)$ & $6007(3)$ & $7276(3)$ & $49(1)$ \\
\hline$C(14)$ & $9421(3)$ & $5779(2)$ & $6050(3)$ & $41(1)$ \\
\hline
\end{tabular}




\begin{tabular}{|c|c|c|c|c|}
\hline$C(15)$ & $7281(2)$ & $9011(2)$ & $3258(2)$ & $29(1)$ \\
\hline C (16) & $8324(2)$ & $8791(2)$ & $3138(2)$ & $30(1)$ \\
\hline C (17) & $8719(2)$ & $9684(2)$ & $2515(2)$ & $32(1)$ \\
\hline$C(18)$ & $7914(3)$ & $10434(2)$ & $2234(2)$ & $35(1)$ \\
\hline C (19) & $7030(2)$ & $10025(2)$ & $2695(2)$ & $33(1)$ \\
\hline$C(20)$ & $6552(3)$ & $8294(3)$ & $3795(3)$ & $43(1)$ \\
\hline$C(21)$ & $8903(3)$ & $7790(2)$ & $3502(3)$ & $44(1)$ \\
\hline C (22) & $9790(3)$ & $9787(3)$ & $2129(3)$ & $48(1)$ \\
\hline C (23) & $8000(3)$ & $11470(3)$ & $1529(3)$ & $55(1)$ \\
\hline$C(24)$ & $6008(3)$ & $10536(3)$ & $2592(3)$ & $54(1)$ \\
\hline C (25) & $6201(2)$ & $5707(2)$ & $2731(2)$ & $24(1)$ \\
\hline$C(26)$ & $5896(2)$ & $4803(2)$ & $2967(2)$ & $30(1)$ \\
\hline$C(27)$ & $5994(2)$ & $4017(2)$ & $3813(2)$ & $33(1)$ \\
\hline C (28) & $6427(2)$ & $4078(2)$ & $4462(2)$ & $34(1)$ \\
\hline C (29) & $6741(2)$ & $4964(2)$ & $4247(2)$ & $29(1)$ \\
\hline$C(30)$ & $6616(2)$ & $5759(2)$ & $3404(2)$ & $25(1)$ \\
\hline C (31) & $5634(3)$ & $3082(2)$ & $4021(3)$ & $52(1)$ \\
\hline C (32) & $7212(3)$ & $5053(2)$ & $4930(2)$ & $42(1)$ \\
\hline$C(33)$ & $4796(2)$ & $7062(2)$ & $2119(2)$ & $21(1)$ \\
\hline C (34) & $4495(2)$ & $7789(2)$ & $2549(2)$ & $23(1)$ \\
\hline$C(35)$ & $3509(2)$ & $8127(2)$ & $2865(2)$ & $24(1)$ \\
\hline$C(36)$ & $2768(2)$ & $7761(2)$ & $2777(2)$ & $25(1)$ \\
\hline C (37) & $3045(2)$ & $7020(2)$ & $2386(2)$ & $24(1)$ \\
\hline$C(38)$ & $4031(2)$ & $6677(2)$ & $2081(2)$ & $24(1)$ \\
\hline$C(39)$ & $3217(2)$ & $8924(2)$ & $3287(2)$ & $35(1)$ \\
\hline$C(40)$ & $2271(2)$ & $6622(2)$ & $2252(2)$ & $34(1)$ \\
\hline$C(41)$ & $6193(2)$ & $6256(2)$ & $865(2)$ & $23(1)$ \\
\hline$C(42)$ & $5593(2)$ & $6533(2)$ & $219(2)$ & $24(1)$ \\
\hline$C(43)$ & $5829(2)$ & $6158(2)$ & $-524(2)$ & $26(1)$ \\
\hline$C(44)$ & $6699(2)$ & $5532(2)$ & $-689(2)$ & $28(1)$ \\
\hline$C(45)$ & $7331(2)$ & $5287(2)$ & $-87(2)$ & $28(1)$ \\
\hline$C(46)$ & $7072(2)$ & $5623(2)$ & $683(2)$ & $26(1)$ \\
\hline$C(47)$ & $5128(2)$ & $6442(2)$ & $-1156(2)$ & $33(1)$ \\
\hline$C(48)$ & $8280(2)$ & $4607(2)$ & $-231(2)$ & $37(1)$ \\
\hline$C(49)$ & $6679(2)$ & $7484(2)$ & $1374(2)$ & $23(1)$ \\
\hline$C(50)$ & $6366(2)$ & $8472(2)$ & $882(2)$ & $30(1)$ \\
\hline C ( 51$)$ & $7004(3)$ & $9176(2)$ & $454(2)$ & $37(1)$ \\
\hline$C(52)$ & $7981(3)$ & $8919(2)$ & $500(2)$ & $38(1)$ \\
\hline$C(53)$ & $8318(2)$ & $7940(2)$ & $970(2)$ & $30(1)$ \\
\hline$C(54)$ & $7681(2)$ & $7243(2)$ & $1397(2)$ & $24(1)$ \\
\hline$C(55)$ & $6625(4)$ & $10209(3)$ & $-97(3)$ & $62(1)$ \\
\hline$C(56)$ & $9386(2)$ & $7640(3)$ & $955(2)$ & $39(1)$ \\
\hline B (1) & $5963(2)$ & $6640(2)$ & $1775(2)$ & $22(1)$ \\
\hline
\end{tabular}




\begin{tabular}{|c|c|}
\hline $\mathrm{Ga}(1)-\mathrm{Fe}(2)$ & $2.2655(10)$ \\
\hline $\mathrm{Ga}(1)-\mathrm{Fe}(1)$ & $2.2722(10)$ \\
\hline $\mathrm{Fe}(1)-\mathrm{C}(2)$ & $1.763(3)$ \\
\hline $\mathrm{Fe}(1)-\mathrm{C}(1)$ & $1.764(3)$ \\
\hline $\mathrm{Fe}(1)-\mathrm{C}(9)$ & $2.106(3)$ \\
\hline $\mathrm{Fe}(1)-\mathrm{C}(6)$ & $2.107(3)$ \\
\hline $\operatorname{Fe}(1)-C(7)$ & $2.113(3)$ \\
\hline $\mathrm{Fe}(1)-\mathrm{C}(8)$ & $2.120(3)$ \\
\hline $\mathrm{Fe}(1)-\mathrm{C}(5)$ & $2.121(3)$ \\
\hline $\mathrm{Fe}(2)-\mathrm{C}(3)$ & $1.770(3)$ \\
\hline $\mathrm{Fe}(2)-\mathrm{C}(4)$ & $1.771(3)$ \\
\hline $\mathrm{Fe}(2)-\mathrm{C}(15)$ & $2.096(3)$ \\
\hline $\operatorname{Fe}(2)-\mathrm{C}(17)$ & $2.100(3)$ \\
\hline $\operatorname{Fe}(2)-\mathrm{C}(19)$ & $2.112(3)$ \\
\hline $\mathrm{Fe}(2)-\mathrm{C}(16)$ & $2.117(3)$ \\
\hline $\mathrm{Fe}(2)-\mathrm{C}(18)$ & $2.117(3)$ \\
\hline$F(1)-C(31)$ & $1.31(2)$ \\
\hline$F(1 A)-C(31)$ & $1.32(4)$ \\
\hline$F(2)-C(31)$ & $1.224(6)$ \\
\hline$F(2 A)-C(31)$ & $1.626(15)$ \\
\hline$F(3)-C(31)$ & $1.445(7)$ \\
\hline$F(3 A)-C(31)$ & $1.236(7)$ \\
\hline$F(4)-C(32)$ & $1.306(11)$ \\
\hline$F(4 A)-C(32)$ & $1.307(9)$ \\
\hline$F(5)-C(32)$ & $1.429(9)$ \\
\hline$F(5 A)-C(32)$ & $1.242(9)$ \\
\hline$F(6)-C(32)$ & $1.262(9)$ \\
\hline$F(6 A)-C(32)$ & $1.442(9)$ \\
\hline$F(7)-C(39)$ & $1.345(4)$ \\
\hline$F(8)-C(39)$ & $1.326(4)$ \\
\hline$F(9)-C(39)$ & $1.335(4)$ \\
\hline$F(10)-C(40)$ & $1.325(4)$ \\
\hline$F(11)-C(40)$ & $1.323(4)$ \\
\hline$F(12)-C(40)$ & $1.341(4)$ \\
\hline$F(13)-C(47)$ & $1.331(4)$ \\
\hline$F(14)-C(47)$ & $1.340(4)$ \\
\hline$F(15)-C(47)$ & $1.337(3)$ \\
\hline$F(16)-C(48)$ & $1.341(4)$ \\
\hline$F(17)-C(48)$ & $1.283(4)$ \\
\hline$F(18)-C(48)$ & $1.322(4)$ \\
\hline$F(19)-C(55)$ & $1.307(5)$ \\
\hline$F(20)-C(55)$ & $1.362(6)$ \\
\hline$F(21)-C(55)$ & $1.270(5)$ \\
\hline$F(22)-C(56)$ & $1.332(4)$ \\
\hline$F(23)-C(56)$ & $1.345(4)$ \\
\hline$F(24)-C(56)$ & $1.338(4)$ \\
\hline $\mathrm{O}(1)-\mathrm{C}(1)$ & $1.150(3)$ \\
\hline $\mathrm{O}(2)-\mathrm{C}(2)$ & $1.140(4)$ \\
\hline $\mathrm{O}(3)-\mathrm{C}(3)$ & $1.145(3)$ \\
\hline $\mathrm{O}(4)-\mathrm{C}(4)$ & $1.141(4)$ \\
\hline$C(5)-C(9)$ & $1.424(4)$ \\
\hline$C(5)-C(6)$ & $1.436(4)$ \\
\hline$C(5)-C(10)$ & $1.504(4)$ \\
\hline$C(6)-C(7)$ & $1.435(4)$ \\
\hline$C(6)-C(11)$ & $1.496(4)$ \\
\hline$C(7)-C(8)$ & $1.413(4)$ \\
\hline$C(7)-C(12)$ & $1.504(4)$ \\
\hline
\end{tabular}




\begin{tabular}{|c|c|}
\hline$C(8)-C(9)$ & $1.441(4)$ \\
\hline$C(8)-C(13)$ & $1.501(5)$ \\
\hline$C(9)-C(14)$ & $1.504(4)$ \\
\hline$C(15)-C(16)$ & $1.425(4)$ \\
\hline$C(15)-C(19)$ & $1.433(4)$ \\
\hline$C(15)-C(20)$ & $1.507(4)$ \\
\hline$C(16)-C(17)$ & $1.435(4)$ \\
\hline$C(16)-C(21)$ & $1.499(4)$ \\
\hline$C(17)-C(18)$ & $1.436(5)$ \\
\hline$C(17)-C(22)$ & $1.497(4)$ \\
\hline$C(18)-C(19)$ & $1.413(4)$ \\
\hline$C(18)-C(23)$ & $1.507(4)$ \\
\hline$C(19)-C(24)$ & $1.504(5)$ \\
\hline$C(25)-C(30)$ & $1.392(4)$ \\
\hline$C(25)-C(26)$ & $1.404(4)$ \\
\hline $\mathrm{C}(25)-\mathrm{B}(1)$ & $1.638(4)$ \\
\hline$C(26)-C(27)$ & $1.386(4)$ \\
\hline$C(27)-C(28)$ & $1.385(4)$ \\
\hline$C(27)-C(31)$ & $1.498(4)$ \\
\hline$C(28)-C(29)$ & $1.393(4)$ \\
\hline$C(29)-C(30)$ & $1.396(4)$ \\
\hline$C(29)-C(32)$ & $1.491(4)$ \\
\hline$C(33)-C(38)$ & $1.398(4)$ \\
\hline$C(33)-C(34)$ & $1.409(4)$ \\
\hline$C(33)-B(1)$ & $1.640(4)$ \\
\hline$C(34)-C(35)$ & $1.385(4)$ \\
\hline$C(35)-C(36)$ & $1.385(4)$ \\
\hline$C(35)-C(39)$ & $1.497(4)$ \\
\hline$C(36)-C(37)$ & $1.388(4)$ \\
\hline$C(37)-C(38)$ & $1.384(4)$ \\
\hline$C(37)-C(40)$ & $1.497(4)$ \\
\hline$C(41)-C(42)$ & $1.398(4)$ \\
\hline$C(41)-C(46)$ & $1.403(4)$ \\
\hline$C(41)-B(1)$ & $1.646(4)$ \\
\hline$C(42)-C(43)$ & $1.394(4)$ \\
\hline$C(43)-C(44)$ & $1.384(4)$ \\
\hline$C(43)-C(47)$ & $1.496(4)$ \\
\hline$C(44)-C(45)$ & $1.390(4)$ \\
\hline$C(45)-C(46)$ & $1.390(4)$ \\
\hline$C(45)-C(48)$ & $1.498(4)$ \\
\hline$C(49)-C(50)$ & $1.396(4)$ \\
\hline$C(49)-C(54)$ & $1.408(4)$ \\
\hline$C(49)-B(1)$ & $1.640(4)$ \\
\hline$C(50)-C(51)$ & $1.398(4)$ \\
\hline$C(51)-C(52)$ & $1.381(5)$ \\
\hline$C(51)-C(55)$ & $1.492(5)$ \\
\hline$C(52)-C(53)$ & $1.386(5)$ \\
\hline$C(53)-C(54)$ & $1.390(4)$ \\
\hline$C(53)-C(56)$ & $1.487(4)$ \\
\hline $\operatorname{Fe}(2)-\mathrm{Ga}(1)-\mathrm{Fe}(1)$ & $178.99(2)$ \\
\hline $\mathrm{C}(2)-\mathrm{Fe}(1)-\mathrm{C}(1)$ & $94.86(13)$ \\
\hline$C(2)-F e(1)-C(9)$ & $93.82(13)$ \\
\hline$C(1)-F e(1)-C(9)$ & $160.44(12)$ \\
\hline$C(2)-F e(1)-C(6)$ & $158.92(12)$ \\
\hline $\mathrm{C}(1)-\mathrm{Fe}(1)-\mathrm{C}(6)$ & $101.85(12)$ \\
\hline$C(9)-F e(1)-C(6)$ & $66.46(12)$ \\
\hline$C(2)-F e(1)-C(7)$ & $126.67(14)$ \\
\hline $\mathrm{C}(1)-\mathrm{Fe}(1)-\mathrm{C}(7)$ & $94.75(12)$ \\
\hline$C(9)-F e(1)-C(7)$ & $66.11(11)$ \\
\hline$C(6)-\mathrm{Fe}(1)-\mathrm{C}(7)$ & $39.77(12)$ \\
\hline
\end{tabular}




\begin{tabular}{|c|c|}
\hline$C(2)-F e(1)-C(8)$ & $93.81(14)$ \\
\hline $\mathrm{C}(1)-\mathrm{Fe}(1)-\mathrm{C}(8)$ & $121.90(12)$ \\
\hline$C(9)-F e(1)-C(8)$ & $39.87(11)$ \\
\hline$C(6)-\mathrm{Fe}(1)-\mathrm{C}(8)$ & $66.47(12)$ \\
\hline $\mathrm{C}(7)-\mathrm{Fe}(1)-\mathrm{C}(8)$ & $39.01(12)$ \\
\hline$C(2)-F e(1)-C(5)$ & $126.87(13)$ \\
\hline$C(1)-\mathrm{Fe}(1)-\mathrm{C}(5)$ & $137.93(12)$ \\
\hline$C(9)-F e(1)-C(5)$ & $39.38(11)$ \\
\hline$C(6)-F e(1)-C(5)$ & $39.71(11)$ \\
\hline$C(7)-\mathrm{Fe}(1)-\mathrm{C}(5)$ & $66.35(11)$ \\
\hline$C(8)-F e(1)-C(5)$ & $66.50(11)$ \\
\hline $\mathrm{C}(2)-\mathrm{Fe}(1)-\mathrm{Ga}(1)$ & $88.71(11)$ \\
\hline $\mathrm{C}(1)-\mathrm{Fe}(1)-\mathrm{Ga}(1)$ & $85.68(9)$ \\
\hline $\mathrm{C}(9)-\mathrm{Fe}(1)-\mathrm{Ga}(1)$ & $112.02(9)$ \\
\hline $\mathrm{C}(6)-\mathrm{Fe}(1)-\mathrm{Ga}(1)$ & $105.15(9)$ \\
\hline $\mathrm{C}(7)-\mathrm{Fe}(1)-\mathrm{Ga}(1)$ & $144.30(9)$ \\
\hline $\mathrm{C}(8)-\mathrm{Fe}(1)-\mathrm{Ga}(1)$ & $151.87(8)$ \\
\hline$C(5)-F e(1)-G a(1)$ & $89.71(8)$ \\
\hline$C(3)-F e(2)-C(4)$ & $95.23(14)$ \\
\hline$C(3)-\operatorname{Fe}(2)-C(15)$ & $157.45(13)$ \\
\hline$C(4)-F e(2)-C(15)$ & $96.03(13)$ \\
\hline$C(3)-F e(2)-C(17)$ & $98.93(13)$ \\
\hline$C(4)-\operatorname{Fe}(2)-C(17)$ & $161.10(13)$ \\
\hline$C(15)-\operatorname{Fe}(2)-C(17)$ & $66.54(12)$ \\
\hline$C(3)-F e(2)-C(19)$ & $119.52(13)$ \\
\hline$C(4)-F e(2)-C(19)$ & $95.53(13)$ \\
\hline$C(15)-\mathrm{Fe}(2)-\mathrm{C}(19)$ & $39.83(12)$ \\
\hline$C(17)-F e(2)-C(19)$ & $66.56(12)$ \\
\hline $\mathrm{C}(3)-\mathrm{Fe}(2)-\mathrm{C}(16)$ & $135.64(13)$ \\
\hline$C(4)-F e(2)-C(16)$ & $128.91(13)$ \\
\hline$C(15)-\operatorname{Fe}(2)-C(16)$ & $39.54(12)$ \\
\hline$C(17)-\mathrm{Fe}(2)-\mathrm{C}(16)$ & $39.79(11)$ \\
\hline$C(19)-\operatorname{Fe}(2)-C(16)$ & $66.68(12)$ \\
\hline$C(3)-F e(2)-C(18)$ & $91.60(13)$ \\
\hline$C(4)-F e(2)-C(18)$ & $127.70(14)$ \\
\hline$C(15)-\mathrm{Fe}(2)-\mathrm{C}(18)$ & $66.10(12)$ \\
\hline $\mathrm{C}(17)-\mathrm{Fe}(2)-\mathrm{C}(18)$ & $39.82(13)$ \\
\hline$C(19)-F e(2)-C(18)$ & $39.04(12)$ \\
\hline$C(16)-\operatorname{Fe}(2)-C(18)$ & $66.50(12)$ \\
\hline $\mathrm{C}(3)-\mathrm{Fe}(2)-\mathrm{Ga}(1)$ & $90.58(10)$ \\
\hline $\mathrm{C}(4)-\mathrm{Fe}(2)-\mathrm{Ga}(1)$ & $88.68(11)$ \\
\hline $\mathrm{C}(15)-\mathrm{Fe}(2)-\mathrm{Ga}(1)$ & $109.13(9)$ \\
\hline $\mathrm{C}(17)-\mathrm{Fe}(2)-\mathrm{Ga}(1)$ & $103.57(9)$ \\
\hline $\mathrm{C}(19)-\mathrm{Fe}(2)-\mathrm{Ga}(1)$ & $148.92(9)$ \\
\hline $\mathrm{C}(16)-\mathrm{Fe}(2)-\mathrm{Ga}(1)$ & $86.78(9)$ \\
\hline $\mathrm{C}(18)-\mathrm{Fe}(2)-\mathrm{Ga}(1)$ & $143.12(10)$ \\
\hline$O(1)-C(1)-\operatorname{Fe}(1)$ & $178.1(2)$ \\
\hline $\mathrm{O}(2)-\mathrm{C}(2)-\mathrm{Fe}(1)$ & $177.0(3)$ \\
\hline$O(3)-C(3)-F e(2)$ & $176.1(3)$ \\
\hline$O(4)-C(4)-\operatorname{Ee}(2)$ & $178.6(3)$ \\
\hline$C(9)-C(5)-C(6)$ & $107.6(2)$ \\
\hline$C(9)-C(5)-C(10)$ & $125.9(3)$ \\
\hline$C(6)-C(5)-C(10)$ & $126.1(3)$ \\
\hline$C(9)-C(5)-F e(1)$ & $69.71(15)$ \\
\hline$C(6)-C(5)-F e(1)$ & $69.60(15)$ \\
\hline$C(10)-C(5)-F e(1)$ & $131.6(2)$ \\
\hline$C(7)-C(6)-C(5)$ & $107.6(3)$ \\
\hline$C(7)-C(6)-C(11)$ & $125.3(3)$ \\
\hline$C(5)-C(6)-C(11)$ & $126.8(3)$ \\
\hline$C(7)-C(6)-\mathrm{Fe}(1)$ & $70.36(16)$ \\
\hline$C(5)-C(6)-F e(1)$ & $70.70(16)$ \\
\hline
\end{tabular}




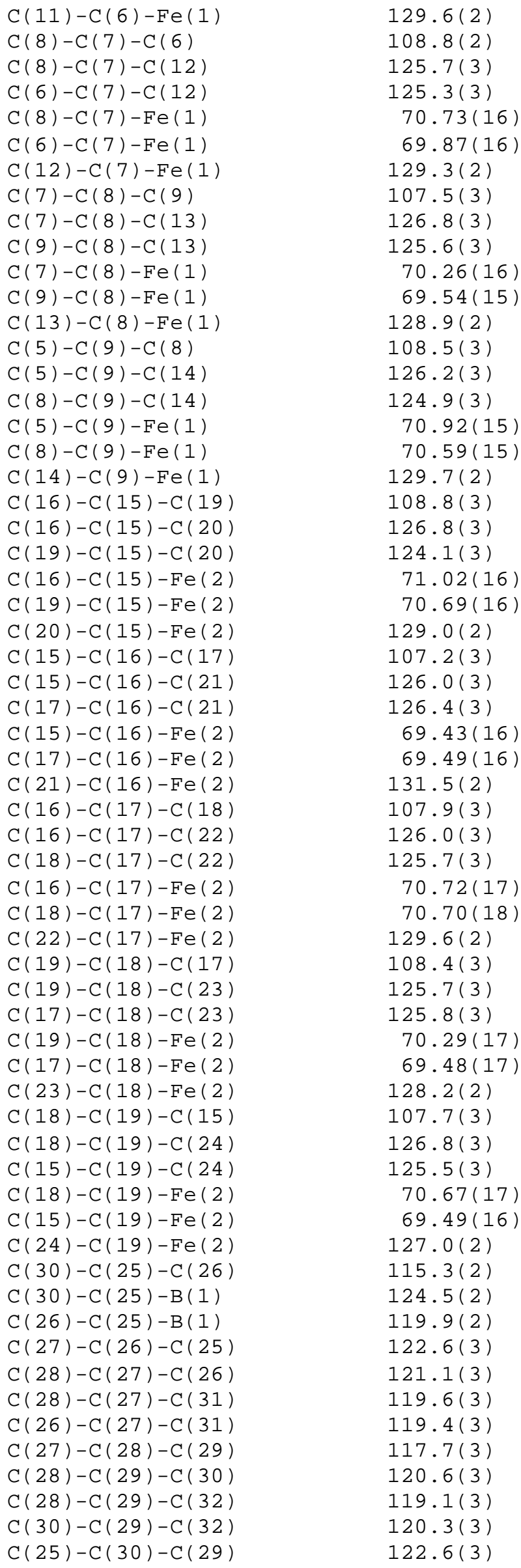




\begin{tabular}{|c|c|}
\hline$F(2)-C(31)-F(3 A)$ & $62.9(7)$ \\
\hline$F(2)-C(31)-F(1)$ & $113.1(11)$ \\
\hline$F(3 A)-C(31)-F(1)$ & $123.2(9)$ \\
\hline$F(2)-C(31)-F(1 A)$ & $114.4(18)$ \\
\hline$F(3 A)-C(31)-F(1 A)$ & $111.2(15)$ \\
\hline$F(1)-C(31)-F(1 A)$ & $13(2)$ \\
\hline$F(2)-C(31)-F(3)$ & $106.3(4)$ \\
\hline$F(3 A)-C(31)-F(3)$ & $43.5(7)$ \\
\hline$F(1)-C(31)-F(3)$ & $103.8(10)$ \\
\hline$F(1 A)-C(31)-F(3)$ & $91.1(16)$ \\
\hline$F(2)-C(31)-C(27)$ & $114.3(3)$ \\
\hline$F(3 A)-C(31)-C(27)$ & $121.9(4)$ \\
\hline$F(1)-C(31)-C(27)$ & $111.0(9)$ \\
\hline$F(1 A)-C(31)-C(27)$ & $119.4(16)$ \\
\hline$F(3)-C(31)-C(27)$ & $107.5(4)$ \\
\hline$F(2)-C(31)-F(2 A)$ & $34.1(3)$ \\
\hline$F(3 A)-C(31)-F(2 A)$ & $95.4(8)$ \\
\hline$F(1)-C(31)-F(2 A)$ & $87.4(11)$ \\
\hline$F(1 A)-C(31)-F(2 A)$ & $94.3(18)$ \\
\hline$F(3)-C(31)-F(2 A)$ & $136.8(4)$ \\
\hline$C(27)-C(31)-F(2 A)$ & $106.9(4)$ \\
\hline$F(5 A)-C(32)-F(6)$ & $71.9(6)$ \\
\hline$F(5 A)-C(32)-F(4 A)$ & $112.5(9)$ \\
\hline$F(6)-C(32)-F(4 A)$ & $120.9(9)$ \\
\hline$F(5 A)-C(32)-F(4)$ & $117.8(8)$ \\
\hline$F(6)-C(32)-F(4)$ & $113.0(10)$ \\
\hline$F(4 A)-C(32)-F(4)$ & $10.3(11)$ \\
\hline$F(5 A)-C(32)-F(5)$ & $32.2(5)$ \\
\hline$F(6)-C(32)-F(5)$ & $103.5(6)$ \\
\hline$F(4 A)-C(32)-F(5)$ & $91.0(7)$ \\
\hline$F(4)-C(32)-F(5)$ & $99.6(7)$ \\
\hline$F(5 A)-C(32)-F(6 A)$ & $103.2(6)$ \\
\hline$F(6)-C(32)-F(6 A)$ & $32.9(6)$ \\
\hline$F(4 A)-C(32)-F(6 A)$ & $99.5(7)$ \\
\hline$F(4)-C(32)-F(6 A)$ & $89.6(7)$ \\
\hline$F(5)-C(32)-F(6 A)$ & $132.3(5)$ \\
\hline$F(5 A)-C(32)-C(29)$ & $116.8(5)$ \\
\hline$F(6)-C(32)-C(29)$ & $115.5(4)$ \\
\hline$F(4 A)-C(32)-C(29)$ & $113.1(6)$ \\
\hline$F(4)-C(32)-C(29)$ & $114.9(6)$ \\
\hline$F(5)-C(32)-C(29)$ & $108.3(6)$ \\
\hline$F(6 A)-C(32)-C(29)$ & $109.8(4)$ \\
\hline$C(38)-C(33)-C(34)$ & $115.2(2)$ \\
\hline $\mathrm{C}(38)-\mathrm{C}(33)-\mathrm{B}(1)$ & $122.7(2)$ \\
\hline $\mathrm{C}(34)-\mathrm{C}(33)-\mathrm{B}(1)$ & $121.9(2)$ \\
\hline$C(35)-C(34)-C(33)$ & $122.0(2)$ \\
\hline$C(34)-C(35)-C(36)$ & $121.4(2)$ \\
\hline$C(34)-C(35)-C(39)$ & $120.4(3)$ \\
\hline$C(36)-C(35)-C(39)$ & $118.1(3)$ \\
\hline$C(35)-C(36)-C(37)$ & $117.7(3)$ \\
\hline$C(38)-C(37)-C(36)$ & $120.7(2)$ \\
\hline$C(38)-C(37)-C(40)$ & $119.6(3)$ \\
\hline$C(36)-C(37)-C(40)$ & $119.6(3)$ \\
\hline$C(37)-C(38)-C(33)$ & $122.8(2)$ \\
\hline$F(8)-C(39)-F(9)$ & $106.9(3)$ \\
\hline$F(8)-C(39)-F(7)$ & $106.5(3)$ \\
\hline$F(9)-C(39)-F(7)$ & $103.9(3)$ \\
\hline$F(8)-C(39)-C(35)$ & $113.4(3)$ \\
\hline$F(9)-C(39)-C(35)$ & $112.4(3)$ \\
\hline$F(7)-C(39)-C(35)$ & $113.1(3)$ \\
\hline$F(11)-C(40)-F(10)$ & $107.5(3)$ \\
\hline
\end{tabular}




\begin{tabular}{|c|c|}
\hline$F(11)-C(40)-F(12)$ & $105.1(3)$ \\
\hline$F(10)-C(40)-F(12)$ & $105.2(3)$ \\
\hline$F(11)-C(40)-C(37)$ & $111.9(3)$ \\
\hline$F(10)-C(40)-C(37)$ & $113.8(3)$ \\
\hline$F(12)-C(40)-C(37)$ & $112.7(3)$ \\
\hline$C(42)-C(41)-C(46)$ & $115.7(3)$ \\
\hline$C(42)-C(41)-B(1)$ & $125.3(2)$ \\
\hline$C(46)-C(41)-B(1)$ & $118.9(2)$ \\
\hline$C(43)-C(42)-C(41)$ & $121.9(3)$ \\
\hline$C(44)-C(43)-C(42)$ & $121.3(3)$ \\
\hline$C(44)-C(43)-C(47)$ & $119.4(3)$ \\
\hline$C(42)-C(43)-C(47)$ & $119.2(3)$ \\
\hline$C(43)-C(44)-C(45)$ & $117.7(3)$ \\
\hline$C(46)-C(45)-C(44)$ & $120.8(3)$ \\
\hline$C(46)-C(45)-C(48)$ & $119.3(3)$ \\
\hline$C(44)-C(45)-C(48)$ & $119.8(3)$ \\
\hline$C(45)-C(46)-C(41)$ & $122.4(3)$ \\
\hline$F(13)-C(47)-F(15)$ & $105.9(3)$ \\
\hline$F(13)-C(47)-F(14)$ & $106.3(3)$ \\
\hline$F(15)-C(47)-F(14)$ & $105.4(2)$ \\
\hline$F(13)-C(47)-C(43)$ & $113.2(2)$ \\
\hline$F(15)-C(47)-C(43)$ & $112.8(2)$ \\
\hline$F(14)-C(47)-C(43)$ & $112.7(3)$ \\
\hline$F(17)-C(48)-F(18)$ & $109.4(3)$ \\
\hline$F(17)-C(48)-F(16)$ & $105.8(3)$ \\
\hline$F(18)-C(48)-F(16)$ & $102.6(3)$ \\
\hline$F(17)-C(48)-C(45)$ & $113.1(3)$ \\
\hline$F(18)-C(48)-C(45)$ & $112.7(3)$ \\
\hline$F(16)-C(48)-C(45)$ & $112.6(3)$ \\
\hline$C(50)-C(49)-C(54)$ & $115.5(2)$ \\
\hline$C(50)-C(49)-B(1)$ & $122.0(2)$ \\
\hline$C(54)-C(49)-B(1)$ & $122.1(2)$ \\
\hline$C(49)-C(50)-C(51)$ & $122.1(3)$ \\
\hline$C(52)-C(51)-C(50)$ & $121.0(3)$ \\
\hline$C(52)-C(51)-C(55)$ & $120.0(3)$ \\
\hline$C(50)-C(51)-C(55)$ & $118.9(3)$ \\
\hline$C(51)-C(52)-C(53)$ & $118.3(3)$ \\
\hline$C(52)-C(53)-C(54)$ & $120.5(3)$ \\
\hline$C(52)-C(53)-C(56)$ & $118.7(3)$ \\
\hline$C(54)-C(53)-C(56)$ & $120.6(3)$ \\
\hline$C(53)-C(54)-C(49)$ & $122.5(3)$ \\
\hline$F(21)-C(55)-F(19)$ & $110.8(4)$ \\
\hline$F(21)-C(55)-F(20)$ & $105.4(4)$ \\
\hline$F(19)-C(55)-F(20)$ & $97.8(4)$ \\
\hline$F(21)-C(55)-C(51)$ & $115.2(4)$ \\
\hline$F(19)-C(55)-C(51)$ & $113.2(4)$ \\
\hline$F(20)-C(55)-C(51)$ & $112.9(3)$ \\
\hline$F(22)-C(56)-F(24)$ & $106.1(3)$ \\
\hline$F(22)-C(56)-F(23)$ & $106.2(3)$ \\
\hline$F(24)-C(56)-F(23)$ & $105.1(3)$ \\
\hline$F(22)-C(56)-C(53)$ & $112.2(3)$ \\
\hline$F(24)-C(56)-C(53)$ & $113.1(3)$ \\
\hline$F(23)-C(56)-C(53)$ & $113.5(3)$ \\
\hline$C(25)-B(1)-C(33)$ & $104.2(2)$ \\
\hline$C(25)-B(1)-C(49)$ & $113.7(2)$ \\
\hline$C(33)-B(1)-C(49)$ & $112.7(2)$ \\
\hline$C(25)-B(1)-C(41)$ & $109.4(2)$ \\
\hline$C(33)-B(1)-C(41)$ & $113.1(2)$ \\
\hline$C(49)-B(1)-C(41)$ & $103.9(2)$ \\
\hline
\end{tabular}


Table 1.4 Anisotropic displacement parameters (A^2 x 10^3) for 7.

The anisotropic displacement factor exponent takes the form:

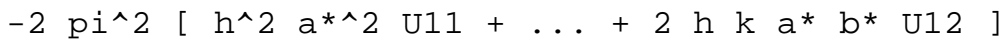

\begin{tabular}{|c|c|c|c|c|c|c|}
\hline & $\mathrm{U} 11$ & U2 2 & U33 & $\mathrm{U} 23$ & U13 & $\mathrm{U} 12$ \\
\hline $\mathrm{Ga}(1)$ & $28(1)$ & $24(1)$ & $22(1)$ & $-6(1)$ & $-8(1)$ & $-6(1)$ \\
\hline $\mathrm{Fe}(1)$ & $24(1)$ & 21 (1) & $19(1)$ & $-4(1)$ & $-4(1)$ & $-7(1)$ \\
\hline $\operatorname{Fe}(2)$ & $23(1)$ & $22(1)$ & $23(1)$ & $-7(1)$ & $-6(1)$ & $-5(1)$ \\
\hline$F(1)$ & $48(6)$ & $31(4)$ & $53(6)$ & $1(4)$ & $-25(5)$ & $-19(3)$ \\
\hline$F(1 A)$ & $34(5)$ & $35(9)$ & $71(16)$ & $0(9)$ & $-19(8)$ & $-16(6)$ \\
\hline$F(2)$ & $46(2)$ & $25(2)$ & $104(9)$ & $-23(4)$ & $-11(3)$ & $-4(2)$ \\
\hline$F(2 A)$ & $62(4)$ & $32(4)$ & $87(9)$ & $-24(5)$ & $-14(5)$ & $-9(3)$ \\
\hline$F(3)$ & $79(4)$ & $53(4)$ & $42(3)$ & $12(2)$ & $-17(3)$ & $-45(3)$ \\
\hline$F(3 A)$ & $115(13)$ & $32(4)$ & $75(10)$ & $19(5)$ & $-57(11)$ & $-33(6)$ \\
\hline$F(4)$ & $36(4)$ & $71(7)$ & $76(6)$ & $-54(6)$ & $-28(4)$ & $15(3)$ \\
\hline$F(4 A)$ & $129(9)$ & $28(3)$ & $68(5)$ & $12(3)$ & $-79(6)$ & $-2(4)$ \\
\hline$F(5)$ & $73(5)$ & $108(8)$ & $24(4)$ & $-30(4)$ & $-5(3)$ & $2(5)$ \\
\hline$F(5 A)$ & $80(5)$ & $120(8)$ & $94(8)$ & $-86(6)$ & $-31(5)$ & $20(5)$ \\
\hline$F(6)$ & $132(9)$ & $38(3)$ & $55(5)$ & $4(3)$ & $-58(6)$ & $-32(5)$ \\
\hline$F(6 A)$ & $81(6)$ & $71(5)$ & $49(4)$ & $1(3)$ & $-37(4)$ & $-38(4)$ \\
\hline$F(7)$ & $85(2)$ & $66(2)$ & $46(1)$ & $-38(1)$ & $-2(1)$ & $-11(1)$ \\
\hline$F(8)$ & $41(1)$ & $56(1)$ & $114(2)$ & $-61(1)$ & $5(1)$ & $-15(1)$ \\
\hline$F(9)$ & $46(1)$ & $36(1)$ & $92(2)$ & $-36(1)$ & $-21(1)$ & $14(1)$ \\
\hline$F(10)$ & $24(1)$ & $88(2)$ & $115(2)$ & $-69(2)$ & $1(1)$ & $-12(1)$ \\
\hline$F(11)$ & $104(2)$ & $107(2)$ & $51(1)$ & $3(1)$ & $-43(1)$ & $-64(2)$ \\
\hline $\mathrm{F}(12)$ & $46(1)$ & $45(1)$ & $76(2)$ & $-28(1)$ & $-13(1)$ & $-18(1)$ \\
\hline$F(13)$ & $29(1)$ & $111(2)$ & $39(1)$ & $-23(1)$ & $-9(1)$ & $-17(1)$ \\
\hline$F(14)$ & $85(2)$ & $54(1)$ & $66(2)$ & $-28(1)$ & $-49(1)$ & $1(1)$ \\
\hline$F(15)$ & $48(1)$ & $44(1)$ & 41 (1) & $10(1)$ & $-23(1)$ & $-9(1)$ \\
\hline$F(16)$ & $51(1)$ & $81(2)$ & $62(2)$ & $-38(1)$ & $-2(1)$ & $16(1)$ \\
\hline$F(17)$ & $66(2)$ & $33(1)$ & $124(2)$ & $6(1)$ & $24(2)$ & $17(1)$ \\
\hline$F(18)$ & $36(1)$ & $122(2)$ & $162(3)$ & $-111(2)$ & $-40(2)$ & $29(1)$ \\
\hline$F(19)$ & $186(4)$ & $68(2)$ & $71(2)$ & $18(2)$ & $-73(2)$ & $2(2)$ \\
\hline$F(20)$ & $135(3)$ & $38(1)$ & $104(2)$ & $0(1)$ & $-48(2)$ & $19(2)$ \\
\hline$F(21)$ & $130(3)$ & $48(2)$ & $261(4)$ & $53(2)$ & $-110(3)$ & $-48(2)$ \\
\hline$F(22)$ & $33(1)$ & $160(3)$ & $57(1)$ & $-73(2)$ & $4(1)$ & $-10(1)$ \\
\hline$F(23)$ & 31 (1) & $66(1)$ & $73(2)$ & $-24(1)$ & $-17(1)$ & $-1(1)$ \\
\hline$F(24)$ & $45(1)$ & $79(2)$ & $90(2)$ & $-44(1)$ & $-19(1)$ & $-22(1)$ \\
\hline$O(1)$ & $37(1)$ & $28(1)$ & $45(1)$ & $-15(1)$ & $-10(1)$ & $-8(1)$ \\
\hline$O(2)$ & $47(2)$ & $49(2)$ & $49(2)$ & $-21(1)$ & $14(1)$ & $-7(1)$ \\
\hline$O(3)$ & $41(1)$ & $29(1)$ & $64(2)$ & $-18(1)$ & $-19(1)$ & $-7(1)$ \\
\hline$O(4)$ & 31 (1) & $98(2)$ & $61(2)$ & $-50(2)$ & $6(1)$ & $-19(1)$ \\
\hline$C(1)$ & $24(1)$ & $25(1)$ & $23(1)$ & $-5(1)$ & $-5(1)$ & $-4(1)$ \\
\hline$C(2)$ & $38(2)$ & $26(1)$ & $29(2)$ & $-8(1)$ & $-2(1)$ & $-8(1)$ \\
\hline$C(3)$ & $26(1)$ & $26(1)$ & $34(2)$ & $-9(1)$ & $-10(1)$ & $0(1)$ \\
\hline$C(4)$ & $27(2)$ & $47(2)$ & $39(2)$ & $-22(2)$ & $-7(1)$ & $-11(1)$ \\
\hline$C(5)$ & $29(1)$ & $23(1)$ & $23(1)$ & $-4(1)$ & $-3(1)$ & $-11(1)$ \\
\hline$C(6)$ & $35(2)$ & $24(1)$ & $21(1)$ & $-4(1)$ & $-1(1)$ & $-13(1)$ \\
\hline$C(7)$ & $43(2)$ & $29(1)$ & $20(1)$ & $1(1)$ & $-8(1)$ & $-18(1)$ \\
\hline$C(8)$ & $33(2)$ & $24(1)$ & $30(2)$ & $2(1)$ & $-12(1)$ & $-9(1)$ \\
\hline$C(9)$ & $34(2)$ & $20(1)$ & $26(1)$ & $-4(1)$ & $-4(1)$ & $-10(1)$ \\
\hline$C(10)$ & $32(2)$ & $42(2)$ & $35(2)$ & $-11(1)$ & $-6(1)$ & $-16(1)$ \\
\hline$C(11)$ & $45(2)$ & $35(2)$ & $42(2)$ & $-17(2)$ & $11(2)$ & $-19(2)$ \\
\hline$C(12)$ & $78(3)$ & $52(2)$ & $28(2)$ & $-5(2)$ & $-18(2)$ & $-31(2)$ \\
\hline$C(13)$ & $43(2)$ & $35(2)$ & $59(2)$ & $4(2)$ & $-25(2)$ & $-4(2)$ \\
\hline$C(14)$ & $46(2)$ & $28(2)$ & $48(2)$ & $-18(1)$ & $-1(2)$ & $-9(1)$ \\
\hline
\end{tabular}




\begin{tabular}{|c|c|c|c|c|c|c|}
\hline $\mathrm{C}(15)$ & $37(2)$ & 31 (1) & $26(1)$ & $-11(1)$ & $-9(1)$ & $-11(1)$ \\
\hline$C(16)$ & $40(2)$ & $31(1)$ & $22(1)$ & $-12(1)$ & $-5(1)$ & $-6(1)$ \\
\hline$C(17)$ & $36(2)$ & $41(2)$ & $23(1)$ & $-15(1)$ & $-2(1)$ & $-12(1)$ \\
\hline C (18) & $48(2)$ & $34(2)$ & $22(1)$ & $-4(1)$ & $-9(1)$ & $-14(1)$ \\
\hline C (19) & $40(2)$ & $36(2)$ & $26(2)$ & $-7(1)$ & $-15(1)$ & $-8(1)$ \\
\hline$C(20)$ & $53(2)$ & $40(2)$ & $44(2)$ & $-14(2)$ & $-11(2)$ & $-24(2)$ \\
\hline $\mathrm{C}(21)$ & $58(2)$ & $36(2)$ & $38(2)$ & $-22(2)$ & $-11(2)$ & $6(2)$ \\
\hline C (22) & $41(2)$ & $66(2)$ & $44(2)$ & $-31(2)$ & $6(2)$ & $-20(2)$ \\
\hline C (23) & $79(3)$ & $43(2)$ & $36(2)$ & $5(2)$ & $-14(2)$ & $-27(2)$ \\
\hline$C(24)$ & $51(2)$ & $59(2)$ & $54(2)$ & $-11(2)$ & $-34(2)$ & $-2(2)$ \\
\hline$C(25)$ & $19(1)$ & $20(1)$ & $25(1)$ & $-4(1)$ & $-4(1)$ & $0(1)$ \\
\hline$C(26)$ & $26(1)$ & $24(1)$ & $36(2)$ & $-3(1)$ & $-14(1)$ & $-4(1)$ \\
\hline$C(27)$ & $26(1)$ & $22(1)$ & $42(2)$ & $1(1)$ & $-12(1)$ & $-7(1)$ \\
\hline$C(28)$ & $33(2)$ & $24(1)$ & $30(2)$ & $6(1)$ & $-10(1)$ & $-2(1)$ \\
\hline$C(29)$ & $33(2)$ & $24(1)$ & $26(1)$ & $-7(1)$ & $-8(1)$ & $2(1)$ \\
\hline$C(30)$ & $29(1)$ & $19(1)$ & $23(1)$ & $-6(1)$ & $-7(1)$ & $2(1)$ \\
\hline$C(31)$ & $46(2)$ & $27(2)$ & $73(3)$ & $11(2)$ & $-34(2)$ & $-14(2)$ \\
\hline$C(32)$ & $66(2)$ & $29(2)$ & $32(2)$ & $-9(1)$ & $-23(2)$ & $4(2)$ \\
\hline$C(33)$ & $24(1)$ & $16(1)$ & $18(1)$ & $0(1)$ & $-8(1)$ & $-1(1)$ \\
\hline$C(34)$ & $24(1)$ & $18(1)$ & $24(1)$ & $-4(1)$ & $-9(1)$ & $-3(1)$ \\
\hline$C(35)$ & $28(1)$ & $20(1)$ & $21(1)$ & $-5(1)$ & $-5(1)$ & $-2(1)$ \\
\hline$C(36)$ & $21(1)$ & $25(1)$ & $22(1)$ & $-4(1)$ & $-3(1)$ & $-2(1)$ \\
\hline C (37) & $23(1)$ & $26(1)$ & $21(1)$ & $-4(1)$ & $-5(1)$ & $-6(1)$ \\
\hline$C(38)$ & $27(1)$ & $21(1)$ & $23(1)$ & $-6(1)$ & $-5(1)$ & $-5(1)$ \\
\hline$C(39)$ & $28(2)$ & $33(2)$ & $44(2)$ & $-20(1)$ & $-2(1)$ & $-2(1)$ \\
\hline C ( 40$)$ & $29(2)$ & $42(2)$ & $35(2)$ & $-15(1)$ & $-6(1)$ & $-11(1)$ \\
\hline$C(41)$ & $22(1)$ & $18(1)$ & $25(1)$ & $-4(1)$ & $-5(1)$ & $-3(1)$ \\
\hline C ( 42$)$ & $23(1)$ & 21 (1) & $23(1)$ & $-3(1)$ & $-5(1)$ & $-3(1)$ \\
\hline$C(43)$ & $26(1)$ & $26(1)$ & $23(1)$ & $-4(1)$ & $-5(1)$ & $-7(1)$ \\
\hline$C(44)$ & $31(2)$ & $25(1)$ & $26(1)$ & $-10(1)$ & $-3(1)$ & $-6(1)$ \\
\hline$C(45)$ & $26(1)$ & $25(1)$ & $32(2)$ & $-12(1)$ & $-4(1)$ & $-3(1)$ \\
\hline$C(46)$ & $26(1)$ & $24(1)$ & $30(2)$ & $-10(1)$ & $-9(1)$ & $-2(1)$ \\
\hline$C(47)$ & $35(2)$ & $36(2)$ & $26(2)$ & $-6(1)$ & $-8(1)$ & $-9(1)$ \\
\hline$C(48)$ & $34(2)$ & $34(2)$ & $45(2)$ & $-22(1)$ & $-8(1)$ & $3(1)$ \\
\hline C (49) & $25(1)$ & $23(1)$ & $17(1)$ & $-5(1)$ & $-5(1)$ & $-4(1)$ \\
\hline$C(50)$ & $33(2)$ & $25(1)$ & $27(2)$ & $-1(1)$ & $-12(1)$ & $-4(1)$ \\
\hline$C(51)$ & $48(2)$ & $29(2)$ & $29(2)$ & $4(1)$ & $-15(1)$ & $-14(1)$ \\
\hline$C(52)$ & $43(2)$ & $38(2)$ & $30(2)$ & $-2(1)$ & $-5(1)$ & $-23(2)$ \\
\hline$C(53)$ & $27(1)$ & $42(2)$ & $25(1)$ & $-15(1)$ & $-2(1)$ & $-11(1)$ \\
\hline C (54) & $24(1)$ & $26(1)$ & $22(1)$ & $-9(1)$ & $-5(1)$ & $-4(1)$ \\
\hline$C(55)$ & $78(3)$ & $32(2)$ & $60(3)$ & $14(2)$ & $-30(2)$ & $-15(2)$ \\
\hline$C(56)$ & $30(2)$ & $60(2)$ & $36(2)$ & $-27(2)$ & $0(1)$ & $-14(2)$ \\
\hline B (1) & $21(1)$ & $19(1)$ & $24(2)$ & $-4(1)$ & $-7(1)$ & $-2(1)$ \\
\hline
\end{tabular}




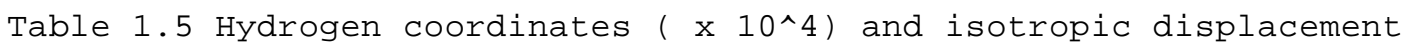
parameters $\left(A^{\wedge} 2 \times 10^{\wedge} 3\right)$ for 7 .

\begin{tabular}{|c|c|c|c|c|}
\hline & $\mathrm{x}$ & $\mathrm{Y}$ & z & $\mathrm{U}(\mathrm{eq})$ \\
\hline $\mathrm{H}(10 \mathrm{~A})$ & 7529 & 6957 & 5792 & 53 \\
\hline $\mathrm{H}(10 \mathrm{~B})$ & 6862 & 7689 & 6341 & 53 \\
\hline $\mathrm{H}(10 \mathrm{C})$ & 6922 & 6524 & 6889 & 53 \\
\hline $\mathrm{H}(11 \mathrm{~A})$ & 6923 & 7713 & 8458 & 64 \\
\hline $\mathrm{H}(11 \mathrm{~B})$ & 6844 & 8461 & 7395 & 64 \\
\hline $\mathrm{H}(11 \mathrm{C})$ & 7518 & 8631 & 7944 & 64 \\
\hline $\mathrm{H}(12 \mathrm{~A})$ & 9010 & 8104 & 8388 & 75 \\
\hline $\mathrm{H}(12 \mathrm{~B})$ & 10067 & 7445 & 8220 & 75 \\
\hline $\mathrm{H}(12 \mathrm{C})$ & 9158 & 6959 & 9035 & 75 \\
\hline $\mathrm{H}(13 \mathrm{~A})$ & 11027 & 6387 & 7429 & 74 \\
\hline $\mathrm{H}(13 \mathrm{~B})$ & 11055 & 5908 & 6667 & 74 \\
\hline $\mathrm{H}(13 \mathrm{C})$ & 10621 & 5366 & 7798 & 74 \\
\hline $\mathrm{H}(14 \mathrm{~A})$ & 9359 & 5103 & 6499 & 61 \\
\hline $\mathrm{H}(14 \mathrm{~B})$ & 10109 & 5817 & 5689 & 61 \\
\hline $\mathrm{H}(14 \mathrm{C})$ & 9002 & 5969 & 5596 & 61 \\
\hline $\mathrm{H}(20 \mathrm{~A})$ & 5923 & 8629 & 4075 & 64 \\
\hline $\mathrm{H}(20 \mathrm{~B})$ & 6808 & 7746 & 4313 & 64 \\
\hline $\mathrm{H}(20 \mathrm{C})$ & 6449 & 8040 & 3346 & 64 \\
\hline $\mathrm{H}(21 \mathrm{~A})$ & 8544 & 7417 & 4148 & 66 \\
\hline $\mathrm{H}(21 \mathrm{~B})$ & 9547 & 7857 & 3536 & 66 \\
\hline $\mathrm{H}(21 \mathrm{C})$ & 8998 & 7440 & 3060 & 66 \\
\hline $\mathrm{H}(22 \mathrm{~A})$ & 10030 & 9686 & 1506 & 72 \\
\hline $\mathrm{H}(22 \mathrm{~B})$ & 10164 & 9294 & 2588 & 72 \\
\hline $\mathrm{H}(22 \mathrm{C})$ & 9877 & 10448 & 2039 & 72 \\
\hline $\mathrm{H}(23 \mathrm{~A})$ & 8089 & 11487 & 871 & 82 \\
\hline $\mathrm{H}(23 \mathrm{~B})$ & 8569 & 11685 & 1565 & 82 \\
\hline $\mathrm{H}(23 \mathrm{C})$ & 7399 & 11911 & 1693 & 82 \\
\hline $\mathrm{H}(24 \mathrm{~A})$ & 5968 & 11242 & 2446 & 81 \\
\hline $\mathrm{H}(24 \mathrm{~B})$ & 5529 & 10262 & 3198 & 81 \\
\hline $\mathrm{H}(24 \mathrm{C})$ & 5860 & 10437 & 2065 & 81 \\
\hline $\mathrm{H}(26)$ & 5612 & 4728 & 2530 & 35 \\
\hline $\mathrm{H}(28)$ & 6507 & 3534 & 5035 & 41 \\
\hline $\mathrm{H}(30)$ & 6823 & 6359 & 3284 & 30 \\
\hline $\mathrm{H}(34)$ & 4983 & 8056 & 2624 & 27 \\
\hline $\mathrm{H}(36)$ & 2093 & 8009 & 2976 & 30 \\
\hline $\mathrm{H}(38)$ & 4196 & 6156 & 1834 & 29 \\
\hline $\mathrm{H}(42)$ & 5007 & 6990 & 289 & 29 \\
\hline $\mathrm{H}(44)$ & 6859 & 5278 & -1196 & 33 \\
\hline $\mathrm{H}(46)$ & 7505 & 5415 & 1101 & 32 \\
\hline $\mathrm{H}(50)$ & 5698 & 8673 & 838 & 36 \\
\hline $\mathrm{H}(52)$ & 8410 & 9400 & 217 & 45 \\
\hline $\mathrm{H}(54)$ & 7932 & 6577 & 1717 & 29 \\
\hline
\end{tabular}




\section{Details of the crystal structure of compound 8}

Table 2.1 Crystal data and structure refinement for $\mathbf{8}$.

Empirical formula

Formula weight

Temperature

Wavelength

Crystal system

Space group

Unit cell dimensions

Volume

Z

Density (calculated)

Absorption coefficient

$\mathrm{F}(000)$

Crystal size

Theta range for data collection

Index ranges

Reflections collected

Independent reflections

Completeness to theta $=25.00^{\circ}$

Absorption correction

Max. and min. transmission

Refinement method

Data / restraints / parameters

Goodness-of-fit on $\mathrm{F}^{2}$

Final $R$ indices [I $>2 \operatorname{sigma}(\mathrm{I})]$

$\mathrm{R}$ indices (all data)

Largest diff. peak and hole

\section{C56 H42 B F24 Fe2 In O4}

1472.23

$150(2) \mathrm{K}$

$0.71073 \AA$

Triclinic

$\mathrm{P}-1$

$\mathrm{a}=14.5327(10) \AA$

$\alpha=65.829(3)^{\circ}$.

$\mathrm{b}=14.6439(10) \AA$

$\beta=68.927(3)^{\circ}$.

$\mathrm{c}=16.2678(13) \AA$

2920.5(4) $\AA^{3}$

2

$1.674 \mathrm{Mg} / \mathrm{m}^{3}$

$1.003 \mathrm{~mm}^{-1}$

1464

$0.35 \times 0.28 \times 0.05 \mathrm{~mm}^{3}$

3.53 to $26.37^{\circ}$.

$-17<=\mathrm{h}<=18,-16<=\mathrm{k}<=18,-16<=\mathrm{l}<=20$

28114

$9917[\mathrm{R}($ int $)=0.1035]$

$86.9 \%$

Semi-empirical from equivalents

0.9515 and 0.7202

Full-matrix least-squares on $\mathrm{F}^{2}$

$9917 / 30 / 788$

1.019

$\mathrm{R} 1=0.0944, \mathrm{wR} 2=0.1918$

$\mathrm{R} 1=0.1812, \mathrm{wR} 2=0.2314$

1.832 and -1.026 e. $\AA^{-3}$ 
Table 2.2 Atomic coordinates ( x 10 $)$ and equivalent isotropic displacement parameters $\left(\AA^{2} \times 10^{3}\right)$ for 8 .

$\mathrm{U}(\mathrm{eq})$ is defined as one third of the trace of the orthogonalized $\mathrm{U}^{\mathrm{ij}}$ tensor.

\begin{tabular}{|c|c|c|c|c|}
\hline & $\mathrm{x}$ & $\mathrm{y}$ & $\mathrm{z}$ & $\mathrm{U}(\mathrm{eq})$ \\
\hline $\mathrm{C}(1)$ & $11639(9)$ & $9673(8)$ & $4760(9)$ & $37(3)$ \\
\hline$C(2)$ & $14610(8)$ & $9048(8)$ & $6226(7)$ & $31(3)$ \\
\hline $\mathrm{C}(3)$ & 13001(8) & 10862(8) & $3706(7)$ & $28(2)$ \\
\hline $\mathrm{C}(4)$ & $15636(10)$ & $7727(8)$ & $5438(8)$ & $36(3)$ \\
\hline$C(5)$ & $13815(9)$ & $9285(10)$ & 2742(9) & $46(3)$ \\
\hline$C(6)$ & $13290(11)$ & $8464(9)$ & $3356(8)$ & $47(3)$ \\
\hline$C(7)$ & 12183(9) & $9805(9)$ & 2761(8) & $38(3)$ \\
\hline $\mathrm{C}(8)$ & $12285(10)$ & $8795(8)$ & $3379(7)$ & $38(3)$ \\
\hline $\mathrm{C}(9)$ & 13132(11) & 10110(9) & $2350(8)$ & $47(3)$ \\
\hline $\mathrm{C}(10)$ & $14227(8)$ & $7145(7)$ & $7757(7)$ & $27(2)$ \\
\hline$C(11)$ & 13301(8) & $7454(7)$ & $7543(7)$ & $31(3)$ \\
\hline$C(12)$ & $14276(8)$ & $6348(7)$ & $6768(7)$ & $29(3)$ \\
\hline$C(13)$ & $13317(8)$ & $6949(7)$ & $6949(7)$ & $28(2)$ \\
\hline $\mathrm{C}(14)$ & $14826(8)$ & $6475(8)$ & $7283(7)$ & $31(3)$ \\
\hline$C(15)$ & 14591(9) & $5624(8)$ & $6256(8)$ & $43(3)$ \\
\hline$C(16)$ & $12451(8)$ & $6936(9)$ & $6646(7)$ & $41(3)$ \\
\hline$C(17)$ & $12442(8)$ & $8093(8)$ & $7965(8)$ & $40(3)$ \\
\hline $\mathrm{C}(18)$ & $14517(10)$ & 7431(9) & $8403(8)$ & $48(3)$ \\
\hline$C(19)$ & 15839(9) & $5902(8)$ & $7371(8)$ & $47(3)$ \\
\hline $\mathrm{C}(20)$ & 13743(13) & $7381(11)$ & $3821(11)$ & $83(5)$ \\
\hline$C(21)$ & $11430(13)$ & $8167(12)$ & $3915(11)$ & $87(6)$ \\
\hline$C(22)$ & 14941(14) & $9300(14)$ & $2409(13)$ & $106(6)$ \\
\hline$C(23)$ & $13390(14)$ & 11101(11) & $1608(9)$ & $86(5)$ \\
\hline $\mathrm{C}(24)$ & $11237(11)$ & $10396(11)$ & $2548(10)$ & $68(4)$ \\
\hline$C(25)$ & $12802(9)$ & $3381(9)$ & $7454(8)$ & $41(3)$ \\
\hline$C(26)$ & $9308(10)$ & $7077(8)$ & $6404(7)$ & $36(3)$ \\
\hline $\mathrm{C}(27)$ & $8262(9)$ & $5075(8)$ & $5130(8)$ & $36(3)$ \\
\hline $\mathrm{C}(28)$ & $5604(10)$ & 2387(9) & $8967(8)$ & $44(3)$ \\
\hline $\mathrm{C}(29)$ & $6830(8)$ & $5404(8)$ & $10339(7)$ & $33(3)$ \\
\hline$C(30)$ & 11629(9) & 974(8) & $6734(7)$ & $35(3)$ \\
\hline $\mathrm{C}(31)$ & 9959(9) & $3414(8)$ & $11092(7)$ & $35(3)$ \\
\hline$C(32)$ & $8593(8)$ & $4320(7)$ & $6675(6)$ & $25(2)$ \\
\hline$C(33)$ & $8527(8)$ & $5155(7)$ & $5874(7)$ & $26(2)$ \\
\hline$C(34)$ & $8750(8)$ & $6072(7)$ & $5779(7)$ & $30(3)$ \\
\hline$C(35)$ & $9025(8)$ & $6119(7)$ & $6486(7)$ & $26(2)$ \\
\hline$C(36)$ & $9066(7)$ & $5285(7)$ & $7277(6)$ & $24(2)$ \\
\hline $\mathrm{C}(37)$ & $8854(8)$ & $4349(7)$ & $7405(7)$ & $25(2)$ \\
\hline $\mathrm{C}(38)$ & $11432(8)$ & $1773(7)$ & $7142(6)$ & $26(2)$ \\
\hline$C(39)$ & $12215(8)$ & $2164(7)$ & $7118(6)$ & $24(2)$ \\
\hline $\mathrm{C}(40)$ & 11977(8) & $2923(7)$ & $7488(7)$ & $27(2)$ \\
\hline $\mathrm{C}(41)$ & 10993(8) & $3269(7)$ & $7881(7)$ & $26(2)$ \\
\hline $\mathrm{C}(42)$ & 10191(7) & $2859(7)$ & $7930(6)$ & $20(2)$ \\
\hline $\mathrm{C}(43)$ & 10447(8) & $2121(7)$ & $7527(7)$ & $26(2)$ \\
\hline $\mathrm{C}(44)$ & $7975(8)$ & $4324(7)$ & $9434(6)$ & $26(2)$ \\
\hline$C(45)$ & $9455(7)$ & $3380(7)$ & $9785(7)$ & $25(2)$ \\
\hline$C(46)$ & $7757(8)$ & $4686(7)$ & 10161(7) & $27(2)$ \\
\hline $\mathrm{C}(47)$ & $9274(8)$ & $3738(8)$ & $10503(7)$ & $31(3)$ \\
\hline $\mathrm{C}(48)$ & $8408(8)$ & $4410(7)$ & $10704(7)$ & $31(3)$ \\
\hline C(49) & $8834(8)$ & $3684(7)$ & $9215(6)$ & $23(2)$ \\
\hline$C(50)$ & $8517(8)$ & $1515(7)$ & $9233(7)$ & $29(2)$ \\
\hline $\mathrm{C}(51)$ & $7300(7)$ & 2761(7) & $8601(6)$ & $22(2)$ \\
\hline$C(52)$ & $6641(8)$ & 2074(8) & $9013(7)$ & $32(3)$ \\
\hline$C(53)$ & $6927(10)$ & 1074(9) & $9539(8)$ & $44(3)$ \\
\hline $\mathrm{C}(54)$ & $7852(9)$ & $802(8)$ & $9644(8)$ & $36(3)$ \\
\hline
\end{tabular}




\begin{tabular}{|c|c|c|c|c|}
\hline$C(55)$ & $8270(8)$ & $2513(7)$ & $8692(6)$ & $24(2)$ \\
\hline$C(56)$ & $8118(14)$ & $-275(13)$ & $10247(12)$ & $81(5)$ \\
\hline $\mathrm{O}(1)$ & $10884(7)$ & $9660(7)$ & $5358(6)$ & $55(2)$ \\
\hline $\mathrm{O}(2)$ & $13168(6)$ & $11652(6)$ & $3571(6)$ & $46(2)$ \\
\hline $\mathrm{O}(3)$ & $16404(7)$ & $7614(6)$ & $4938(6)$ & $53(2)$ \\
\hline $\mathrm{O}(4)$ & $14728(6)$ & $9823(5)$ & $6186(5)$ & $37(2)$ \\
\hline $\mathrm{F}(1)$ & $9185(5)$ & $7855(4)$ & $5645(4)$ & $50(2)$ \\
\hline $\mathrm{F}(2)$ & $8764(5)$ & $7365(4)$ & $7144(5)$ & $48(2)$ \\
\hline $\mathrm{F}(3)$ & $10264(5)$ & $6958(4)$ & $6389(4)$ & $43(2)$ \\
\hline $\mathrm{F}(4)$ & $12553(5)$ & $4306(6)$ & $7453(6)$ & $73(2)$ \\
\hline $\mathrm{F}(5)$ & $13580(6)$ & $3413(7)$ & $6709(7)$ & $89(3)$ \\
\hline $\mathrm{F}(6)$ & $13160(7)$ & $2882(7)$ & $8176(7)$ & $93(3)$ \\
\hline $\mathrm{F}(7)$ & 6041(6) & $5150(6)$ & $10315(7)$ & $82(3)$ \\
\hline $\mathrm{F}(8)$ & $6552(7)$ & $5494(7)$ & $11165(6)$ & $92(3)$ \\
\hline $\mathrm{F}(9)$ & $6905(7)$ & $6312(6)$ & $9708(6)$ & $88(3)$ \\
\hline $\mathrm{F}(10)$ & 4997(6) & $2686(7)$ & $9666(6)$ & $79(3)$ \\
\hline $\mathrm{F}(11)$ & $5500(6)$ & $3130(7)$ & $8186(6)$ & $93(3)$ \\
\hline $\mathrm{F}(12)$ & $5167(6)$ & $1651(7)$ & $9041(8)$ & $99(3)$ \\
\hline$F(13)$ & $8664(7)$ & $4263(6)$ & $4920(6)$ & $86(3)$ \\
\hline $\mathrm{F}(14)$ & $7300(9)$ & $4967(9)$ & $5383(7)$ & $112(3)$ \\
\hline$F(16)$ & $12561(5)$ & $495(5)$ & $6618(5)$ & $48(2)$ \\
\hline$F(17)$ & $11493(7)$ & $1348(5)$ & $5871(5)$ & $73(3)$ \\
\hline$F(18)$ & $11032(5)$ & $261(5)$ & $7237(5)$ & $57(2)$ \\
\hline $\mathrm{F}(19)$ & $10907(5)$ & $3260(6)$ & $10601(5)$ & $58(2)$ \\
\hline$F(20)$ & $9776(5)$ & $2535(5)$ & 11791(4) & $51(2)$ \\
\hline $\mathrm{F}(21)$ & $9907(6)$ & $4064(5)$ & $11489(5)$ & $59(2)$ \\
\hline$F(23)$ & $9003(13)$ & $-673(12)$ & $9795(11)$ & $176(6)$ \\
\hline$F(24)$ & $8696(11)$ & $-337(10)$ & $10730(10)$ & $150(5)$ \\
\hline $\mathrm{Fe}(1)$ & 12741(1) & $9695(1)$ & $3834(1)$ & $29(1)$ \\
\hline $\mathrm{Fe}(2)$ & $14471(1)$ & $7870(1)$ & $6279(1)$ & $27(1)$ \\
\hline $\operatorname{In}(1)$ & $13655(1)$ & $8833(1)$ & $5020(1)$ & $32(1)$ \\
\hline $\mathrm{B}(1)$ & $9045(9)$ & $3332(8)$ & $8319(8)$ & $24(3)$ \\
\hline $\mathrm{F}(15 \mathrm{~A})$ & $7912(14)$ & $5925(12)$ & $4564(11)$ & $54(4)$ \\
\hline $\mathrm{F}(15 \mathrm{~B})$ & $8652(10)$ & $5724(8)$ & $4268(8)$ & $47(3)$ \\
\hline $\mathrm{F}(22 \mathrm{~A})$ & $7743(12)$ & $-935(11)$ & $10241(10)$ & $69(4)$ \\
\hline$F(22 B)$ & $7441(15)$ & $-569(14)$ & $11035(14)$ & $85(5)$ \\
\hline
\end{tabular}


Table 2.3 Bond lengths $[\AA]$ and angles $\left[{ }^{\circ}\right]$ for $\mathbf{8}$.

\begin{tabular}{|c|c|}
\hline $\mathrm{C}(1)-\mathrm{O}(1)$ & $1.174(13)$ \\
\hline $\mathrm{C}(1)-\mathrm{Fe}(1)$ & $1.757(13)$ \\
\hline $\mathrm{C}(2)-\mathrm{O}(4)$ & $1.164(12)$ \\
\hline $\mathrm{C}(2)-\mathrm{Fe}(2)$ & $1.752(12)$ \\
\hline $\mathrm{C}(3)-\mathrm{O}(2)$ & $1.160(11)$ \\
\hline$C(3)-F e(1)$ & $1.759(11)$ \\
\hline $\mathrm{C}(4)-\mathrm{O}(3)$ & $1.138(13)$ \\
\hline $\mathrm{C}(4)-\mathrm{Fe}(2)$ & $1.779(13)$ \\
\hline$C(5)-C(6)$ & $1.404(17)$ \\
\hline$C(5)-C(9)$ & $1.431(17)$ \\
\hline$C(5)-C(22)$ & $1.53(2)$ \\
\hline $\mathrm{C}(5)-\mathrm{Fe}(1)$ & $2.084(12)$ \\
\hline$C(6)-C(8)$ & $1.405(17)$ \\
\hline$C(6)-C(20)$ & $1.533(18)$ \\
\hline $\mathrm{C}(6)-\mathrm{Fe}(1)$ & $2.106(11)$ \\
\hline$C(7)-C(9)$ & $1.402(16)$ \\
\hline $\mathrm{C}(7)-\mathrm{C}(8)$ & $1.412(15)$ \\
\hline$C(7)-C(24)$ & $1.495(17)$ \\
\hline $\mathrm{C}(7)-\mathrm{Fe}(1)$ & $2.110(10)$ \\
\hline $\mathrm{C}(8)-\mathrm{C}(21)$ & $1.522(17)$ \\
\hline $\mathrm{C}(8)-\mathrm{Fe}(1)$ & $2.082(10)$ \\
\hline$C(9)-C(23)$ & $1.492(16)$ \\
\hline $\mathrm{C}(9)-\mathrm{Fe}(1)$ & $2.125(11)$ \\
\hline $\mathrm{C}(10)-\mathrm{C}(14)$ & $1.417(15)$ \\
\hline $\mathrm{C}(10)-\mathrm{C}(11)$ & $1.423(14)$ \\
\hline $\mathrm{C}(10)-\mathrm{C}(18)$ & $1.493(15)$ \\
\hline $\mathrm{C}(10)-\mathrm{Fe}(2)$ & $2.128(9)$ \\
\hline $\mathrm{C}(11)-\mathrm{C}(13)$ & $1.428(14)$ \\
\hline $\mathrm{C}(11)-\mathrm{C}(17)$ & $1.485(15)$ \\
\hline $\mathrm{C}(11)-\mathrm{Fe}(2)$ & $2.119(10)$ \\
\hline$C(12)-C(14)$ & $1.436(14)$ \\
\hline $\mathrm{C}(12)-\mathrm{C}(13)$ & $1.441(14)$ \\
\hline $\mathrm{C}(12)-\mathrm{C}(15)$ & $1.490(15)$ \\
\hline $\mathrm{C}(12)-\mathrm{Fe}(2)$ & $2.097(9)$ \\
\hline$C(13)-C(16)$ & $1.515(15)$ \\
\hline $\mathrm{C}(13)-\mathrm{Fe}(2)$ & $2.118(10)$ \\
\hline $\mathrm{C}(14)-\mathrm{C}(19)$ & $1.517(15)$ \\
\hline $\mathrm{C}(14)-\mathrm{Fe}(2)$ & $2.104(10)$ \\
\hline$C(25)-F(4)$ & $1.307(13)$ \\
\hline$C(25)-F(6)$ & $1.311(13)$ \\
\hline$C(25)-F(5)$ & $1.321(13)$ \\
\hline $\mathrm{C}(25)-\mathrm{C}(40)$ & $1.495(15)$ \\
\hline$C(26)-F(1)$ & $1.325(11)$ \\
\hline$C(26)-F(2)$ & $1.345(12)$ \\
\hline$C(26)-F(3)$ & $1.347(13)$ \\
\hline $\mathrm{C}(26)-\mathrm{C}(35)$ & $1.504(14)$ \\
\hline $\mathrm{C}(27)-\mathrm{F}(13)$ & $1.303(12)$ \\
\hline$C(27)-F(15 A)$ & $1.312(18)$ \\
\hline $\mathrm{C}(27)-\mathrm{F}(14)$ & $1.340(14)$ \\
\hline $\mathrm{C}(27)-\mathrm{F}(15 \mathrm{~B})$ & $1.345(15)$ \\
\hline $\mathrm{C}(27)-\mathrm{C}(33)$ & $1.449(14)$ \\
\hline $\mathrm{C}(28)-\mathrm{F}(11)$ & $1.319(13)$ \\
\hline $\mathrm{C}(28)-\mathrm{F}(10)$ & $1.322(13)$ \\
\hline $\mathrm{C}(28)-\mathrm{F}(12)$ & $1.332(14)$ \\
\hline $\mathrm{C}(28)-\mathrm{C}(52)$ & $1.476(16)$ \\
\hline$C(29)-F(9)$ & $1.308(12)$ \\
\hline$C(29)-F(8)$ & $1.309(12)$ \\
\hline
\end{tabular}




\begin{tabular}{|c|c|}
\hline $\mathrm{C}(29)-\mathrm{F}(7)$ & $1.313(13)$ \\
\hline$C(29)-C(46)$ & $1.494(14)$ \\
\hline $\mathrm{C}(30)-\mathrm{F}(18)$ & $1.332(13)$ \\
\hline $\mathrm{C}(30)-\mathrm{F}(16)$ & $1.338(12)$ \\
\hline $\mathrm{C}(30)-\mathrm{F}(17)$ & $1.351(12)$ \\
\hline $\mathrm{C}(30)-\mathrm{C}(38)$ & $1.489(14)$ \\
\hline $\mathrm{C}(31)-\mathrm{F}(21)$ & $1.325(12)$ \\
\hline $\mathrm{C}(31)-\mathrm{F}(19)$ & $1.334(13)$ \\
\hline $\mathrm{C}(31)-\mathrm{F}(20)$ & $1.336(11)$ \\
\hline $\mathrm{C}(31)-\mathrm{C}(47)$ & $1.485(15)$ \\
\hline $\mathrm{C}(32)-\mathrm{C}(33)$ & $1.387(12)$ \\
\hline $\mathrm{C}(32)-\mathrm{C}(37)$ & $1.389(13)$ \\
\hline $\mathrm{C}(33)-\mathrm{C}(34)$ & $1.397(13)$ \\
\hline $\mathrm{C}(34)-\mathrm{C}(35)$ & $1.378(13)$ \\
\hline $\mathrm{C}(35)-\mathrm{C}(36)$ & $1.373(12)$ \\
\hline $\mathrm{C}(36)-\mathrm{C}(37)$ & $1.398(13)$ \\
\hline $\mathrm{C}(37)-\mathrm{B}(1)$ & $1.665(13)$ \\
\hline $\mathrm{C}(38)-\mathrm{C}(39)$ & $1.383(14)$ \\
\hline $\mathrm{C}(38)-\mathrm{C}(43)$ & $1.392(14)$ \\
\hline$C(39)-C(40)$ & $1.382(13)$ \\
\hline $\mathrm{C}(40)-\mathrm{C}(41)$ & $1.393(14)$ \\
\hline$C(41)-C(42)$ & $1.408(13)$ \\
\hline $\mathrm{C}(42)-\mathrm{C}(43)$ & $1.389(13)$ \\
\hline $\mathrm{C}(42)-\mathrm{B}(1)$ & $1.628(16)$ \\
\hline $\mathrm{C}(44)-\mathrm{C}(49)$ & $1.380(13)$ \\
\hline $\mathrm{C}(44)-\mathrm{C}(46)$ & $1.391(13)$ \\
\hline$C(45)-C(47)$ & $1.385(14)$ \\
\hline$C(45)-C(49)$ & $1.388(14)$ \\
\hline $\mathrm{C}(46)-\mathrm{C}(48)$ & $1.396(14)$ \\
\hline $\mathrm{C}(47)-\mathrm{C}(48)$ & $1.407(15)$ \\
\hline $\mathrm{C}(49)-\mathrm{B}(1)$ & $1.642(15)$ \\
\hline$C(50)-C(55)$ & $1.393(13)$ \\
\hline$C(50)-C(54)$ & $1.401(14)$ \\
\hline $\mathrm{C}(51)-\mathrm{C}(52)$ & $1.368(13)$ \\
\hline $\mathrm{C}(51)-\mathrm{C}(55)$ & $1.407(14)$ \\
\hline$C(52)-C(53)$ & $1.400(15)$ \\
\hline $\mathrm{C}(53)-\mathrm{C}(54)$ & $1.352(16)$ \\
\hline$C(54)-C(56)$ & $1.511(18)$ \\
\hline $\mathrm{C}(55)-\mathrm{B}(1)$ & $1.641(15)$ \\
\hline$C(56)-F(22 A)$ & $1.23(2)$ \\
\hline $\mathrm{C}(56)-\mathrm{F}(22 \mathrm{~B})$ & $1.29(2)$ \\
\hline $\mathrm{C}(56)-\mathrm{F}(24)$ & $1.307(19)$ \\
\hline$C(56)-F(23)$ & $1.35(2)$ \\
\hline $\mathrm{F}(14)-\mathrm{F}(15 \mathrm{~A})$ & $1.675(19)$ \\
\hline $\mathrm{F}(23)-\mathrm{F}(24)$ & $1.663(19)$ \\
\hline $\mathrm{Fe}(1)-\operatorname{In}(1)$ & $2.4596(17)$ \\
\hline $\mathrm{Fe}(2)-\operatorname{In}(1)$ & $2.4687(17)$ \\
\hline $\mathrm{O}(1)-\mathrm{C}(1)-\mathrm{Fe}(1)$ & $177.5(10)$ \\
\hline $\mathrm{O}(4)-\mathrm{C}(2)-\mathrm{Fe}(2)$ & $178.3(10)$ \\
\hline $\mathrm{O}(2)-\mathrm{C}(3)-\mathrm{Fe}(1)$ & 176.1(9) \\
\hline $\mathrm{O}(3)-\mathrm{C}(4)-\mathrm{Fe}(2)$ & $176.2(10)$ \\
\hline$C(6)-C(5)-C(9)$ & $108.5(11)$ \\
\hline$C(6)-C(5)-C(22)$ & $128.0(14)$ \\
\hline$C(9)-C(5)-C(22)$ & $123.0(13)$ \\
\hline $\mathrm{C}(6)-\mathrm{C}(5)-\mathrm{Fe}(1)$ & 71.3(7) \\
\hline $\mathrm{C}(9)-\mathrm{C}(5)-\mathrm{Fe}(1)$ & $71.7(7)$ \\
\hline $\mathrm{C}(22)-\mathrm{C}(5)-\mathrm{Fe}(1)$ & $129.2(10)$ \\
\hline$C(5)-C(6)-C(8)$ & $107.0(10)$ \\
\hline$C(5)-C(6)-C(20)$ & $126.4(14)$ \\
\hline
\end{tabular}




\begin{tabular}{|c|c|}
\hline$C(8)-C(6)-C(20)$ & $126.3(13)$ \\
\hline $\mathrm{C}(5)-\mathrm{C}(6)-\mathrm{Fe}(1)$ & $69.6(7)$ \\
\hline $\mathrm{C}(8)-\mathrm{C}(6)-\mathrm{Fe}(1)$ & $69.5(6)$ \\
\hline $\mathrm{C}(20)-\mathrm{C}(6)-\mathrm{Fe}(1)$ & 131.4(9) \\
\hline$C(9)-C(7)-C(8)$ & $107.4(11)$ \\
\hline$C(9)-C(7)-C(24)$ & 127.2(12) \\
\hline $\mathrm{C}(8)-\mathrm{C}(7)-\mathrm{C}(24)$ & $125.2(12)$ \\
\hline $\mathrm{C}(9)-\mathrm{C}(7)-\mathrm{Fe}(1)$ & $71.3(6)$ \\
\hline $\mathrm{C}(8)-\mathrm{C}(7)-\mathrm{Fe}(1)$ & $69.3(6)$ \\
\hline $\mathrm{C}(24)-\mathrm{C}(7)-\mathrm{Fe}(1)$ & $128.0(8)$ \\
\hline$C(6)-C(8)-C(7)$ & $109.5(10)$ \\
\hline$C(6)-C(8)-C(21)$ & $126.8(12)$ \\
\hline $\mathrm{C}(7)-\mathrm{C}(8)-\mathrm{C}(21)$ & $123.6(13)$ \\
\hline $\mathrm{C}(6)-\mathrm{C}(8)-\mathrm{Fe}(1)$ & 71.3(7) \\
\hline $\mathrm{C}(7)-\mathrm{C}(8)-\mathrm{Fe}(1)$ & $71.4(6)$ \\
\hline $\mathrm{C}(21)-\mathrm{C}(8)-\mathrm{Fe}(1)$ & 127.1(8) \\
\hline$C(7)-C(9)-C(5)$ & $107.6(10)$ \\
\hline $\mathrm{C}(7)-\mathrm{C}(9)-\mathrm{C}(23)$ & 126.1(13) \\
\hline$C(5)-C(9)-C(23)$ & $126.2(13)$ \\
\hline $\mathrm{C}(7)-\mathrm{C}(9)-\mathrm{Fe}(1)$ & $70.1(6)$ \\
\hline $\mathrm{C}(5)-\mathrm{C}(9)-\mathrm{Fe}(1)$ & $68.6(7)$ \\
\hline $\mathrm{C}(23)-\mathrm{C}(9)-\mathrm{Fe}(1)$ & $128.6(9)$ \\
\hline$C(14)-C(10)-C(11)$ & 108.4(9) \\
\hline$C(14)-C(10)-C(18)$ & $124.9(10)$ \\
\hline $\mathrm{C}(11)-\mathrm{C}(10)-\mathrm{C}(18)$ & $126.7(10)$ \\
\hline $\mathrm{C}(14)-\mathrm{C}(10)-\mathrm{Fe}(2)$ & $69.5(6)$ \\
\hline $\mathrm{C}(11)-\mathrm{C}(10)-\mathrm{Fe}(2)$ & $70.1(6)$ \\
\hline $\mathrm{C}(18)-\mathrm{C}(10)-\mathrm{Fe}(2)$ & $128.2(7)$ \\
\hline $\mathrm{C}(10)-\mathrm{C}(11)-\mathrm{C}(13)$ & 107.9(9) \\
\hline$C(10)-C(11)-C(17)$ & $124.7(10)$ \\
\hline $\mathrm{C}(13)-\mathrm{C}(11)-\mathrm{C}(17)$ & $127.0(10)$ \\
\hline $\mathrm{C}(10)-\mathrm{C}(11)-\mathrm{Fe}(2)$ & $70.8(6)$ \\
\hline $\mathrm{C}(13)-\mathrm{C}(11)-\mathrm{Fe}(2)$ & $70.3(6)$ \\
\hline $\mathrm{C}(17)-\mathrm{C}(11)-\mathrm{Fe}(2)$ & $130.0(7)$ \\
\hline $\mathrm{C}(14)-\mathrm{C}(12)-\mathrm{C}(13)$ & 106.8(9) \\
\hline$C(14)-C(12)-C(15)$ & $125.9(10)$ \\
\hline$C(13)-C(12)-C(15)$ & $126.8(9)$ \\
\hline $\mathrm{C}(14)-\mathrm{C}(12)-\mathrm{Fe}(2)$ & $70.3(5)$ \\
\hline $\mathrm{C}(13)-\mathrm{C}(12)-\mathrm{Fe}(2)$ & $70.8(5)$ \\
\hline $\mathrm{C}(15)-\mathrm{C}(12)-\mathrm{Fe}(2)$ & $130.0(8)$ \\
\hline $\mathrm{C}(11)-\mathrm{C}(13)-\mathrm{C}(12)$ & 108.2(9) \\
\hline$C(11)-C(13)-C(16)$ & $126.8(10)$ \\
\hline$C(12)-C(13)-C(16)$ & 124.7(9) \\
\hline $\mathrm{C}(11)-\mathrm{C}(13)-\mathrm{Fe}(2)$ & $70.3(6)$ \\
\hline $\mathrm{C}(12)-\mathrm{C}(13)-\mathrm{Fe}(2)$ & $69.2(5)$ \\
\hline $\mathrm{C}(16)-\mathrm{C}(13)-\mathrm{Fe}(2)$ & 131.3(7) \\
\hline$C(10)-C(14)-C(12)$ & 108.6(9) \\
\hline$C(10)-C(14)-C(19)$ & $126.2(10)$ \\
\hline$C(12)-C(14)-C(19)$ & 124.9(10) \\
\hline $\mathrm{C}(10)-\mathrm{C}(14)-\mathrm{Fe}(2)$ & $71.4(6)$ \\
\hline $\mathrm{C}(12)-\mathrm{C}(14)-\mathrm{Fe}(2)$ & $69.8(5)$ \\
\hline $\mathrm{C}(19)-\mathrm{C}(14)-\mathrm{Fe}(2)$ & $129.2(7)$ \\
\hline $\mathrm{F}(4)-\mathrm{C}(25)-\mathrm{F}(6)$ & $104.5(10)$ \\
\hline $\mathrm{F}(4)-\mathrm{C}(25)-\mathrm{F}(5)$ & $105.5(9)$ \\
\hline $\mathrm{F}(6)-\mathrm{C}(25)-\mathrm{F}(5)$ & $104.9(11)$ \\
\hline $\mathrm{F}(4)-\mathrm{C}(25)-\mathrm{C}(40)$ & 114.6(10) \\
\hline$F(6)-C(25)-C(40)$ & $113.0(9)$ \\
\hline $\mathrm{F}(5)-\mathrm{C}(25)-\mathrm{C}(40)$ & $113.5(10)$ \\
\hline $\mathrm{F}(1)-\mathrm{C}(26)-\mathrm{F}(2)$ & 106.2(9) \\
\hline $\mathrm{F}(1)-\mathrm{C}(26)-\mathrm{F}(3)$ & 107.4(9) \\
\hline
\end{tabular}




\begin{tabular}{|c|c|}
\hline $\mathrm{F}(2)-\mathrm{C}(26)-\mathrm{F}(3)$ & $105.8(8)$ \\
\hline$F(1)-C(26)-C(35)$ & 113.6(9) \\
\hline $\mathrm{F}(2)-\mathrm{C}(26)-\mathrm{C}(35)$ & $111.6(9)$ \\
\hline $\mathrm{F}(3)-\mathrm{C}(26)-\mathrm{C}(35)$ & 111.7(9) \\
\hline $\mathrm{F}(13)-\mathrm{C}(27)-\mathrm{F}(15 \mathrm{~A})$ & $124.6(12)$ \\
\hline $\mathrm{F}(13)-\mathrm{C}(27)-\mathrm{F}(14)$ & $99.2(9)$ \\
\hline $\mathrm{F}(15 \mathrm{~A})-\mathrm{C}(27)-\mathrm{F}(14)$ & $78.3(11)$ \\
\hline $\mathrm{F}(13)-\mathrm{C}(27)-\mathrm{F}(15 \mathrm{~B})$ & $95.4(10)$ \\
\hline $\mathrm{F}(15 \mathrm{~A})-\mathrm{C}(27)-\mathrm{F}(15 \mathrm{~B})$ & $45.4(8)$ \\
\hline $\mathrm{F}(14)-\mathrm{C}(27)-\mathrm{F}(15 \mathrm{~B})$ & $118.2(11)$ \\
\hline $\mathrm{F}(13)-\mathrm{C}(27)-\mathrm{C}(33)$ & $116.0(10)$ \\
\hline $\mathrm{F}(15 \mathrm{~A})-\mathrm{C}(27)-\mathrm{C}(33)$ & $115.9(11)$ \\
\hline $\mathrm{F}(14)-\mathrm{C}(27)-\mathrm{C}(33)$ & $112.0(10)$ \\
\hline $\mathrm{F}(15 \mathrm{~B})-\mathrm{C}(27)-\mathrm{C}(33)$ & $114.2(10)$ \\
\hline $\mathrm{F}(11)-\mathrm{C}(28)-\mathrm{F}(10)$ & 106.1(11) \\
\hline $\mathrm{F}(11)-\mathrm{C}(28)-\mathrm{F}(12)$ & $105.6(11)$ \\
\hline $\mathrm{F}(10)-\mathrm{C}(28)-\mathrm{F}(12)$ & $102.4(10)$ \\
\hline $\mathrm{F}(11)-\mathrm{C}(28)-\mathrm{C}(52)$ & 114.1(10) \\
\hline $\mathrm{F}(10)-\mathrm{C}(28)-\mathrm{C}(52)$ & $113.3(10)$ \\
\hline $\mathrm{F}(12)-\mathrm{C}(28)-\mathrm{C}(52)$ & $114.3(10)$ \\
\hline $\mathrm{F}(9)-\mathrm{C}(29)-\mathrm{F}(8)$ & $107.2(10)$ \\
\hline $\mathrm{F}(9)-\mathrm{C}(29)-\mathrm{F}(7)$ & $104.6(10)$ \\
\hline $\mathrm{F}(8)-\mathrm{C}(29)-\mathrm{F}(7)$ & 103.2(9) \\
\hline $\mathrm{F}(9)-\mathrm{C}(29)-\mathrm{C}(46)$ & 112.3(9) \\
\hline $\mathrm{F}(8)-\mathrm{C}(29)-\mathrm{C}(46)$ & $114.7(9)$ \\
\hline $\mathrm{F}(7)-\mathrm{C}(29)-\mathrm{C}(46)$ & $114.0(10)$ \\
\hline $\mathrm{F}(18)-\mathrm{C}(30)-\mathrm{F}(16)$ & $106.5(8)$ \\
\hline $\mathrm{F}(18)-\mathrm{C}(30)-\mathrm{F}(17)$ & 104.7(9) \\
\hline $\mathrm{F}(16)-\mathrm{C}(30)-\mathrm{F}(17)$ & 104.9(9) \\
\hline $\mathrm{F}(18)-\mathrm{C}(30)-\mathrm{C}(38)$ & $113.8(9)$ \\
\hline $\mathrm{F}(16)-\mathrm{C}(30)-\mathrm{C}(38)$ & 113.7(9) \\
\hline $\mathrm{F}(17)-\mathrm{C}(30)-\mathrm{C}(38)$ & $112.3(8)$ \\
\hline $\mathrm{F}(21)-\mathrm{C}(31)-\mathrm{F}(19)$ & $107.0(9)$ \\
\hline $\mathrm{F}(21)-\mathrm{C}(31)-\mathrm{F}(20)$ & $106.5(8)$ \\
\hline $\mathrm{F}(19)-\mathrm{C}(31)-\mathrm{F}(20)$ & $105.5(9)$ \\
\hline $\mathrm{F}(21)-\mathrm{C}(31)-\mathrm{C}(47)$ & 114.1(9) \\
\hline $\mathrm{F}(19)-\mathrm{C}(31)-\mathrm{C}(47)$ & 111.9(9) \\
\hline $\mathrm{F}(20)-\mathrm{C}(31)-\mathrm{C}(47)$ & 111.3(9) \\
\hline $\mathrm{C}(33)-\mathrm{C}(32)-\mathrm{C}(37)$ & 123.3(9) \\
\hline $\mathrm{C}(32)-\mathrm{C}(33)-\mathrm{C}(34)$ & 119.0(9) \\
\hline $\mathrm{C}(32)-\mathrm{C}(33)-\mathrm{C}(27)$ & $120.8(9)$ \\
\hline $\mathrm{C}(34)-\mathrm{C}(33)-\mathrm{C}(27)$ & $120.1(8)$ \\
\hline $\mathrm{C}(35)-\mathrm{C}(34)-\mathrm{C}(33)$ & 118.9(8) \\
\hline $\mathrm{C}(36)-\mathrm{C}(35)-\mathrm{C}(34)$ & $120.6(9)$ \\
\hline$C(36)-C(35)-C(26)$ & 118.2(9) \\
\hline $\mathrm{C}(34)-\mathrm{C}(35)-\mathrm{C}(26)$ & 121.1(8) \\
\hline $\mathrm{C}(35)-\mathrm{C}(36)-\mathrm{C}(37)$ & 122.7(9) \\
\hline $\mathrm{C}(32)-\mathrm{C}(37)-\mathrm{C}(36)$ & $115.4(8)$ \\
\hline $\mathrm{C}(32)-\mathrm{C}(37)-\mathrm{B}(1)$ & $123.7(8)$ \\
\hline $\mathrm{C}(36)-\mathrm{C}(37)-\mathrm{B}(1)$ & $120.5(8)$ \\
\hline $\mathrm{C}(39)-\mathrm{C}(38)-\mathrm{C}(43)$ & $121.6(9)$ \\
\hline $\mathrm{C}(39)-\mathrm{C}(38)-\mathrm{C}(30)$ & $120.3(10)$ \\
\hline $\mathrm{C}(43)-\mathrm{C}(38)-\mathrm{C}(30)$ & 118.1(10) \\
\hline $\mathrm{C}(40)-\mathrm{C}(39)-\mathrm{C}(38)$ & 117.2(9) \\
\hline$C(39)-C(40)-C(41)$ & $121.5(9)$ \\
\hline $\mathrm{C}(39)-\mathrm{C}(40)-\mathrm{C}(25)$ & $118.7(10)$ \\
\hline $\mathrm{C}(41)-\mathrm{C}(40)-\mathrm{C}(25)$ & $119.8(10)$ \\
\hline $\mathrm{C}(40)-\mathrm{C}(41)-\mathrm{C}(42)$ & 121.8(9) \\
\hline $\mathrm{C}(43)-\mathrm{C}(42)-\mathrm{C}(41)$ & $115.6(9)$ \\
\hline $\mathrm{C}(43)-\mathrm{C}(42)-\mathrm{B}(1)$ & $123.0(9)$ \\
\hline
\end{tabular}




\begin{tabular}{|c|c|}
\hline $\mathrm{C}(41)-\mathrm{C}(42)-\mathrm{B}(1)$ & $121.0(8)$ \\
\hline $\mathrm{C}(42)-\mathrm{C}(43)-\mathrm{C}(38)$ & 122.3(9) \\
\hline$C(49)-C(44)-C(46)$ & 122.2(9) \\
\hline $\mathrm{C}(47)-\mathrm{C}(45)-\mathrm{C}(49)$ & $122.9(10)$ \\
\hline $\mathrm{C}(44)-\mathrm{C}(46)-\mathrm{C}(48)$ & $120.8(9)$ \\
\hline $\mathrm{C}(44)-\mathrm{C}(46)-\mathrm{C}(29)$ & 119.4(9) \\
\hline $\mathrm{C}(48)-\mathrm{C}(46)-\mathrm{C}(29)$ & 119.8(9) \\
\hline $\mathrm{C}(45)-\mathrm{C}(47)-\mathrm{C}(48)$ & 119.9(9) \\
\hline$C(45)-C(47)-C(31)$ & $121.9(10)$ \\
\hline $\mathrm{C}(48)-\mathrm{C}(47)-\mathrm{C}(31)$ & $118.2(10)$ \\
\hline $\mathrm{C}(46)-\mathrm{C}(48)-\mathrm{C}(47)$ & $117.5(10)$ \\
\hline $\mathrm{C}(44)-\mathrm{C}(49)-\mathrm{C}(45)$ & $116.5(9)$ \\
\hline $\mathrm{C}(44)-\mathrm{C}(49)-\mathrm{B}(1)$ & $118.6(8)$ \\
\hline $\mathrm{C}(45)-\mathrm{C}(49)-\mathrm{B}(1)$ & $124.9(9)$ \\
\hline $\mathrm{C}(55)-\mathrm{C}(50)-\mathrm{C}(54)$ & $122.4(10)$ \\
\hline $\mathrm{C}(52)-\mathrm{C}(51)-\mathrm{C}(55)$ & 122.7(9) \\
\hline$C(51)-C(52)-C(53)$ & $120.0(10)$ \\
\hline $\mathrm{C}(51)-\mathrm{C}(52)-\mathrm{C}(28)$ & $120.8(10)$ \\
\hline $\mathrm{C}(53)-\mathrm{C}(52)-\mathrm{C}(28)$ & 119.1(10) \\
\hline $\mathrm{C}(54)-\mathrm{C}(53)-\mathrm{C}(52)$ & $119.3(10)$ \\
\hline $\mathrm{C}(53)-\mathrm{C}(54)-\mathrm{C}(50)$ & $120.3(10)$ \\
\hline $\mathrm{C}(53)-\mathrm{C}(54)-\mathrm{C}(56)$ & $117.6(11)$ \\
\hline $\mathrm{C}(50)-\mathrm{C}(54)-\mathrm{C}(56)$ & $122.0(12)$ \\
\hline$C(50)-C(55)-C(51)$ & 115.3(9) \\
\hline $\mathrm{C}(50)-\mathrm{C}(55)-\mathrm{B}(1)$ & $119.6(9)$ \\
\hline $\mathrm{C}(51)-\mathrm{C}(55)-\mathrm{B}(1)$ & $124.7(8)$ \\
\hline $\mathrm{F}(22 \mathrm{~A})-\mathrm{C}(56)-\mathrm{F}(22 \mathrm{~B})$ & 71.7(15) \\
\hline $\mathrm{F}(22 \mathrm{~A})-\mathrm{C}(56)-\mathrm{F}(24)$ & $130.4(17)$ \\
\hline $\mathrm{F}(22 \mathrm{~B})-\mathrm{C}(56)-\mathrm{F}(24)$ & $87.5(15)$ \\
\hline $\mathrm{F}(22 \mathrm{~A})-\mathrm{C}(56)-\mathrm{F}(23)$ & $88.0(15)$ \\
\hline $\mathrm{F}(22 \mathrm{~B})-\mathrm{C}(56)-\mathrm{F}(23)$ & $137.3(18)$ \\
\hline $\mathrm{F}(24)-\mathrm{C}(56)-\mathrm{F}(23)$ & $77.6(13)$ \\
\hline $\mathrm{F}(22 \mathrm{~A})-\mathrm{C}(56)-\mathrm{C}(54)$ & $117.4(15)$ \\
\hline $\mathrm{F}(22 \mathrm{~B})-\mathrm{C}(56)-\mathrm{C}(54)$ & $112.6(16)$ \\
\hline$F(24)-C(56)-C(54)$ & $112.1(15)$ \\
\hline $\mathrm{F}(23)-\mathrm{C}(56)-\mathrm{C}(54)$ & $110.1(15)$ \\
\hline $\mathrm{C}(27)-\mathrm{F}(14)-\mathrm{F}(15 \mathrm{~A})$ & 50.1(9) \\
\hline $\mathrm{C}(56)-\mathrm{F}(23)-\mathrm{F}(24)$ & $50.1(10)$ \\
\hline $\mathrm{C}(56)-\mathrm{F}(24)-\mathrm{F}(23)$ & $52.2(10)$ \\
\hline $\mathrm{C}(1)-\mathrm{Fe}(1)-\mathrm{C}(3)$ & $95.8(5)$ \\
\hline $\mathrm{C}(1)-\mathrm{Fe}(1)-\mathrm{C}(8)$ & $93.0(5)$ \\
\hline $\mathrm{C}(3)-\mathrm{Fe}(1)-\mathrm{C}(8)$ & $151.9(4)$ \\
\hline $\mathrm{C}(1)-\mathrm{Fe}(1)-\mathrm{C}(5)$ & $158.0(5)$ \\
\hline $\mathrm{C}(3)-\mathrm{Fe}(1)-\mathrm{C}(5)$ & $105.5(5)$ \\
\hline $\mathrm{C}(8)-\mathrm{Fe}(1)-\mathrm{C}(5)$ & $65.6(5)$ \\
\hline $\mathrm{C}(1)-\mathrm{Fe}(1)-\mathrm{C}(6)$ & $120.2(5)$ \\
\hline $\mathrm{C}(3)-\mathrm{Fe}(1)-\mathrm{C}(6)$ & $143.9(5)$ \\
\hline $\mathrm{C}(8)-\mathrm{Fe}(1)-\mathrm{C}(6)$ & $39.2(5)$ \\
\hline $\mathrm{C}(5)-\mathrm{Fe}(1)-\mathrm{C}(6)$ & $39.2(5)$ \\
\hline $\mathrm{C}(1)-\mathrm{Fe}(1)-\mathrm{C}(7)$ & $101.0(5)$ \\
\hline $\mathrm{C}(3)-\mathrm{Fe}(1)-\mathrm{C}(7)$ & $112.6(4)$ \\
\hline $\mathrm{C}(8)-\mathrm{Fe}(1)-\mathrm{C}(7)$ & $39.4(4)$ \\
\hline $\mathrm{C}(5)-\mathrm{Fe}(1)-\mathrm{C}(7)$ & $66.1(5)$ \\
\hline $\mathrm{C}(6)-\mathrm{Fe}(1)-\mathrm{C}(7)$ & $66.1(5)$ \\
\hline $\mathrm{C}(1)-\mathrm{Fe}(1)-\mathrm{C}(9)$ & $136.8(5)$ \\
\hline $\mathrm{C}(3)-\mathrm{Fe}(1)-\mathrm{C}(9)$ & $90.6(4)$ \\
\hline $\mathrm{C}(8)-\mathrm{Fe}(1)-\mathrm{C}(9)$ & $65.2(4)$ \\
\hline $\mathrm{C}(5)-\mathrm{Fe}(1)-\mathrm{C}(9)$ & $39.7(5)$ \\
\hline $\mathrm{C}(6)-\mathrm{Fe}(1)-\mathrm{C}(9)$ & $65.9(5)$ \\
\hline $\mathrm{C}(7)-\mathrm{Fe}(1)-\mathrm{C}(9)$ & $38.7(4)$ \\
\hline
\end{tabular}




\begin{tabular}{|c|c|}
\hline $\mathrm{C}(1)-\mathrm{Fe}(1)-\operatorname{In}(1)$ & $88.1(4)$ \\
\hline $\mathrm{C}(3)-\mathrm{Fe}(1)-\operatorname{In}(1)$ & $89.6(3)$ \\
\hline $\mathrm{C}(8)-\mathrm{Fe}(1)-\operatorname{In}(1)$ & $117.4(3)$ \\
\hline $\mathrm{C}(5)-\mathrm{Fe}(1)-\operatorname{In}(1)$ & $97.2(4)$ \\
\hline $\mathrm{C}(6)-\mathrm{Fe}(1)-\operatorname{In}(1)$ & $88.8(3)$ \\
\hline $\mathrm{C}(7)-\mathrm{Fe}(1)-\operatorname{In}(1)$ & $154.7(3)$ \\
\hline $\mathrm{C}(9)-\mathrm{Fe}(1)-\operatorname{In}(1)$ & $134.7(4)$ \\
\hline $\mathrm{C}(2)-\mathrm{Fe}(2)-\mathrm{C}(4)$ & $94.8(5)$ \\
\hline $\mathrm{C}(2)-\mathrm{Fe}(2)-\mathrm{C}(12)$ & $162.7(4)$ \\
\hline $\mathrm{C}(4)-\mathrm{Fe}(2)-\mathrm{C}(12)$ & $94.2(5)$ \\
\hline $\mathrm{C}(2)-\mathrm{Fe}(2)-\mathrm{C}(14)$ & $124.9(4)$ \\
\hline $\mathrm{C}(4)-\mathrm{Fe}(2)-\mathrm{C}(14)$ & $91.9(5)$ \\
\hline $\mathrm{C}(12)-\mathrm{Fe}(2)-\mathrm{C}(14)$ & $40.0(4)$ \\
\hline $\mathrm{C}(2)-\mathrm{Fe}(2)-\mathrm{C}(13)$ & $135.3(5)$ \\
\hline $\mathrm{C}(4)-\mathrm{Fe}(2)-\mathrm{C}(13)$ & $129.6(5)$ \\
\hline $\mathrm{C}(12)-\mathrm{Fe}(2)-\mathrm{C}(13)$ & $40.0(4)$ \\
\hline $\mathrm{C}(14)-\mathrm{Fe}(2)-\mathrm{C}(13)$ & $66.3(4)$ \\
\hline $\mathrm{C}(2)-\mathrm{Fe}(2)-\mathrm{C}(11)$ & $100.8(4)$ \\
\hline $\mathrm{C}(4)-\mathrm{Fe}(2)-\mathrm{C}(11)$ & $157.7(4)$ \\
\hline$C(12)-F e(2)-C(11)$ & $66.9(4)$ \\
\hline $\mathrm{C}(14)-\mathrm{Fe}(2)-\mathrm{C}(11)$ & $66.1(4)$ \\
\hline $\mathrm{C}(13)-\mathrm{Fe}(2)-\mathrm{C}(11)$ & $39.4(4)$ \\
\hline $\mathrm{C}(2)-\mathrm{Fe}(2)-\mathrm{C}(10)$ & $96.2(4)$ \\
\hline $\mathrm{C}(4)-\mathrm{Fe}(2)-\mathrm{C}(10)$ & $123.6(5)$ \\
\hline $\mathrm{C}(12)-\mathrm{Fe}(2)-\mathrm{C}(10)$ & $66.5(4)$ \\
\hline $\mathrm{C}(14)-\mathrm{Fe}(2)-\mathrm{C}(10)$ & $39.1(4)$ \\
\hline $\mathrm{C}(13)-\mathrm{Fe}(2)-\mathrm{C}(10)$ & $65.8(4)$ \\
\hline $\mathrm{C}(11)-\mathrm{Fe}(2)-\mathrm{C}(10)$ & $39.1(4)$ \\
\hline $\mathrm{C}(2)-\mathrm{Fe}(2)-\operatorname{In}(1)$ & $86.0(3)$ \\
\hline $\mathrm{C}(4)-\mathrm{Fe}(2)-\operatorname{In}(1)$ & 91.1(3) \\
\hline $\mathrm{C}(12)-\mathrm{Fe}(2)-\operatorname{In}(1)$ & $108.6(3)$ \\
\hline $\mathrm{C}(14)-\mathrm{Fe}(2)-\operatorname{In}(1)$ & $148.6(3)$ \\
\hline $\mathrm{C}(13)-\mathrm{Fe}(2)-\operatorname{In}(1)$ & $88.0(3)$ \\
\hline $\mathrm{C}(11)-\mathrm{Fe}(2)-\operatorname{In}(1)$ & $105.7(3)$ \\
\hline$C(10)-F e(2)-\operatorname{In}(1)$ & $144.7(3)$ \\
\hline $\mathrm{Fe}(1)-\operatorname{In}(1)-\mathrm{Fe}(2)$ & $175.32(6)$ \\
\hline $\mathrm{C}(42)-\mathrm{B}(1)-\mathrm{C}(55)$ & $111.8(8)$ \\
\hline $\mathrm{C}(42)-\mathrm{B}(1)-\mathrm{C}(49)$ & $114.6(8)$ \\
\hline $\mathrm{C}(55)-\mathrm{B}(1)-\mathrm{C}(49)$ & $104.8(8)$ \\
\hline $\mathrm{C}(42)-\mathrm{B}(1)-\mathrm{C}(37)$ & $103.7(8)$ \\
\hline $\mathrm{C}(55)-\mathrm{B}(1)-\mathrm{C}(37)$ & $113.5(8)$ \\
\hline $\mathrm{C}(49)-\mathrm{B}(1)-\mathrm{C}(37)$ & $108.7(8)$ \\
\hline $\mathrm{C}(27)-\mathrm{F}(15 \mathrm{~A})-\mathrm{F}(14)$ & $51.6(8)$ \\
\hline
\end{tabular}

Symmetry transformations used to generate equivalent atoms: 
Table 2.4 Anisotropic displacement parameters $\left(\AA^{2} \mathrm{x} 10^{3}\right)$ for 8 .

The anisotropic displacement factor exponent takes the form: $-2 \pi^{2}\left[h^{2} a^{* 2} U^{11}+\ldots+2 h k a^{*} b^{*} U^{12}\right]$

\begin{tabular}{|c|c|c|c|c|c|c|}
\hline & $\mathrm{U}^{11}$ & $\mathrm{U}^{22}$ & $\mathrm{U}^{33}$ & $\mathrm{U}^{23}$ & $\mathrm{U}^{13}$ & $\mathrm{U}^{12}$ \\
\hline $\mathrm{C}(1)$ & $34(8)$ & $33(6)$ & $50(8)$ & $-14(5)$ & $-20(6)$ & $-6(6)$ \\
\hline$C(2)$ & $24(7)$ & $36(7)$ & $24(6)$ & $-2(5)$ & $-4(5)$ & $-7(5)$ \\
\hline $\mathrm{C}(3)$ & $18(6)$ & $36(7)$ & $28(6)$ & $-11(5)$ & $-9(5)$ & $2(5)$ \\
\hline $\mathrm{C}(4)$ & $42(9)$ & $37(7)$ & $27(6)$ & $-2(5)$ & $-14(6)$ & $-13(6)$ \\
\hline$C(5)$ & $26(8)$ & $74(9)$ & $51(8)$ & $-38(7)$ & $-3(6)$ & $-9(7)$ \\
\hline$C(6)$ & $65(10)$ & $38(7)$ & $40(7)$ & $-21(6)$ & $-26(7)$ & $19(7)$ \\
\hline$C(7)$ & $37(8)$ & $50(7)$ & $33(7)$ & $-12(5)$ & $-20(6)$ & $-4(6)$ \\
\hline $\mathrm{C}(8)$ & $54(9)$ & $36(7)$ & $32(7)$ & $-15(5)$ & $-9(6)$ & $-17(6)$ \\
\hline $\mathrm{C}(9)$ & $71(11)$ & $46(8)$ & $29(7)$ & $-10(5)$ & $-9(6)$ & $-28(7)$ \\
\hline$C(10)$ & $30(7)$ & $13(5)$ & $26(6)$ & $4(4)$ & $-3(5)$ & $-7(5)$ \\
\hline$C(11)$ & $32(7)$ & $27(6)$ & $24(6)$ & $-1(4)$ & $-5(5)$ & $-7(5)$ \\
\hline$C(12)$ & $33(7)$ & $16(5)$ & $38(6)$ & $-5(4)$ & $-11(5)$ & $-6(5)$ \\
\hline$C(13)$ & $27(7)$ & $27(6)$ & $23(6)$ & $-3(4)$ & $-2(5)$ & $-10(5)$ \\
\hline $\mathrm{C}(14)$ & $22(7)$ & $36(6)$ & $30(6)$ & $0(5)$ & $-12(5)$ & $-10(5)$ \\
\hline$C(15)$ & $29(8)$ & $37(7)$ & $61(8)$ & $-17(6)$ & $-8(6)$ & $-6(6)$ \\
\hline$C(16)$ & $28(7)$ & $52(7)$ & $29(6)$ & $-2(5)$ & $1(5)$ & $-17(6)$ \\
\hline$C(17)$ & $31(8)$ & $32(6)$ & $49(7)$ & $-15(5)$ & $3(6)$ & $-9(5)$ \\
\hline $\mathrm{C}(18)$ & $56(9)$ & $52(8)$ & $36(7)$ & $-13(6)$ & $-16(6)$ & $-9(7)$ \\
\hline$C(19)$ & $55(9)$ & $31(6)$ & $42(7)$ & $-3(5)$ & $-15(6)$ & $0(6)$ \\
\hline$C(20)$ & $92(7)$ & $81(6)$ & $77(6)$ & $-35(5)$ & $-28(5)$ & $5(4)$ \\
\hline $\mathrm{C}(21)$ & $114(15)$ & $98(12)$ & $80(11)$ & $-46(10)$ & $-6(10)$ & $-68(11)$ \\
\hline $\mathrm{C}(22)$ & $105(8)$ & $114(8)$ & $105(7)$ & $-46(5)$ & $-27(5)$ & $-13(5)$ \\
\hline$C(23)$ & $130(16)$ & $82(11)$ & $39(8)$ & $-4(7)$ & $2(9)$ & $-67(11)$ \\
\hline $\mathrm{C}(24)$ & $73(11)$ & $91(11)$ & $61(9)$ & $-43(8)$ & $-41(8)$ & 12(9) \\
\hline $\mathrm{C}(25)$ & $42(9)$ & $44(8)$ & $28(7)$ & $1(5)$ & $-11(6)$ & $-12(6)$ \\
\hline$C(26)$ & $55(9)$ & $30(6)$ & $20(6)$ & $1(5)$ & $-14(6)$ & $-10(6)$ \\
\hline$C(27)$ & $44(8)$ & $22(6)$ & $40(7)$ & $-2(5)$ & $-16(6)$ & $-11(5)$ \\
\hline$C(28)$ & $41(9)$ & $53(8)$ & $32(7)$ & $-8(6)$ & $-6(6)$ & $-12(7)$ \\
\hline $\mathrm{C}(29)$ & $22(7)$ & $43(7)$ & $24(6)$ & $-7(5)$ & $-3(5)$ & $0(5)$ \\
\hline$C(30)$ & $38(8)$ & $37(7)$ & $33(7)$ & $-16(5)$ & $-18(6)$ & $11(6)$ \\
\hline $\mathrm{C}(31)$ & $39(8)$ & $32(6)$ & $29(6)$ & $-5(5)$ & $-9(6)$ & $-8(5)$ \\
\hline$C(32)$ & $36(7)$ & $17(5)$ & $17(5)$ & $-4(4)$ & $-7(5)$ & $0(5)$ \\
\hline$C(33)$ & $31(7)$ & $25(6)$ & $21(6)$ & $-9(4)$ & $-7(5)$ & $0(5)$ \\
\hline$C(34)$ & $34(7)$ & $20(5)$ & $27(6)$ & $6(4)$ & $-15(5)$ & $-5(5)$ \\
\hline$C(35)$ & $26(7)$ & $25(5)$ & $28(6)$ & $-8(4)$ & $-12(5)$ & $-2(5)$ \\
\hline$C(36)$ & $23(6)$ & $25(5)$ & $21(5)$ & $-2(4)$ & $-11(5)$ & $-2(5)$ \\
\hline$C(37)$ & $22(6)$ & $21(5)$ & $30(6)$ & $-9(4)$ & $-8(5)$ & $1(4)$ \\
\hline $\mathrm{C}(38)$ & $40(8)$ & $16(5)$ & $16(5)$ & $-2(4)$ & $-9(5)$ & $1(5)$ \\
\hline C(39) & $19(6)$ & $28(6)$ & $12(5)$ & $2(4)$ & $0(4)$ & $-2(5)$ \\
\hline $\mathrm{C}(40)$ & $29(7)$ & $23(5)$ & $28(6)$ & $-3(4)$ & $-9(5)$ & $-11(5)$ \\
\hline $\mathrm{C}(41)$ & $28(7)$ & $24(5)$ & $26(6)$ & $-8(4)$ & $-11(5)$ & $-1(5)$ \\
\hline $\mathrm{C}(42)$ & $28(7)$ & $21(5)$ & $9(5)$ & $-6(4)$ & $-8(4)$ & $6(4)$ \\
\hline$C(43)$ & $21(7)$ & $23(5)$ & $30(6)$ & $-1(4)$ & $-14(5)$ & $0(5)$ \\
\hline$C(44)$ & $25(7)$ & $31(6)$ & $20(5)$ & $-8(4)$ & $-11(5)$ & $4(5)$ \\
\hline$C(45)$ & $14(6)$ & $25(5)$ & $27(6)$ & $-4(4)$ & $-3(5)$ & $-1(4)$ \\
\hline$C(46)$ & $19(6)$ & $27(6)$ & $26(6)$ & $-8(4)$ & $-3(5)$ & $3(5)$ \\
\hline $\mathrm{C}(47)$ & $25(7)$ & $31(6)$ & $25(6)$ & $1(5)$ & $-2(5)$ & $-11(5)$ \\
\hline $\mathrm{C}(48)$ & $40(8)$ & $27(6)$ & $20(6)$ & $-2(4)$ & $-7(5)$ & $-9(5)$ \\
\hline C(49) & $26(7)$ & $18(5)$ & $13(5)$ & $3(4)$ & $-3(4)$ & $-2(5)$ \\
\hline $\mathrm{C}(50)$ & $24(7)$ & $30(6)$ & $26(6)$ & $-3(4)$ & $-9(5)$ & $1(5)$ \\
\hline $\mathrm{C}(51)$ & $23(6)$ & $21(5)$ & $17(5)$ & $-4(4)$ & $0(4)$ & $-7(5)$ \\
\hline$C(52)$ & $25(7)$ & $42(7)$ & $27(6)$ & $-10(5)$ & $-10(5)$ & $-4(5)$ \\
\hline $\mathrm{C}(53)$ & $48(9)$ & $46(7)$ & $34(7)$ & $-9(5)$ & $2(6)$ & $-27(7)$ \\
\hline$C(54)$ & $34(8)$ & $30(6)$ & $39(7)$ & $0(5)$ & $-16(6)$ & $-7(5)$ \\
\hline
\end{tabular}




$\begin{array}{lcccccc}\mathrm{C}(55) & 34(7) & 16(5) & 21(5) & -6(4) & -9(5) & -2(5) \\ \mathrm{C}(56) & 78(7) & 84(6) & 79(6) & -23(4) & -30(5) & -10(5) \\ \mathrm{O}(1) & 34(6) & 79(6) & 55(6) & -31(5) & -3(5) & -11(5) \\ \mathrm{O}(2) & 57(6) & 28(4) & 61(6) & -13(4) & -26(4) & -9(4) \\ \mathrm{O}(3) & 37(6) & 69(6) & 37(5) & -18(4) & 13(4) & -16(5) \\ \mathrm{O}(4) & 41(5) & 24(4) & 42(5) & -1(3) & -16(4) & -9(4) \\ \mathrm{F}(1) & 78(6) & 27(3) & 47(4) & 6(3) & -35(4) & -16(3) \\ \mathrm{F}(2) & 61(5) & 36(4) & 54(4) & -21(3) & -18(4) & -6(3) \\ \mathrm{F}(3) & 38(4) & 43(4) & 53(4) & -12(3) & -18(3) & -16(3) \\ \mathrm{F}(4) & 40(5) & 55(5) & 134(7) & -48(5) & -15(5) & -10(4) \\ \mathrm{F}(5) & 43(5) & 122(7) & 114(7) & -72(6) & 23(5) & -44(5) \\ \mathrm{F}(7) & 36(5) & 86(6) & 143(8) & -72(6) & -27(5) & 13(4) \\ \mathrm{F}(8) & 76(7) & 127(8) & 84(6) & -77(6) & -39(5) & 61(6) \\ \mathrm{F}(9) & 69(4) & 56(4) & 91(4) & -9(3) & 0(3) & 8(3) \\ \mathrm{F}(10) & 35(5) & 132(8) & 82(6) & -72(6) & -15(4) & 23(5) \\ \mathrm{F}(11) & 37(5) & 144(8) & 58(5) & 16(5) & -21(4) & -23(5) \\ \mathrm{F}(12) & 57(6) & 97(7) & 186(10) & -75(7) & -57(6) & -9(5) \\ \mathrm{F}(13) & 125(8) & 80(6) & 87(6) & -61(5) & -64(6) & 29(5) \\ \mathrm{F}(14) & 111(5) & 154(6) & 103(5) & -65(4) & -43(4) & -15(4) \\ \mathrm{F}(16) & 37(5) & 38(4) & 60(4) & -23(3) & -5(3) & 5(3) \\ \mathrm{F}(17) & 125(8) & 49(4) & 62(5) & -33(4) & -54(5) & 20(5) \\ \mathrm{F}(18) & 43(5) & 43(4) & 83(5) & -33(4) & 3(4) & -14(4) \\ \mathrm{F}(19) & 23(4) & 103(6) & 45(4) & -21(4) & -11(3) & -10(4) \\ \mathrm{F}(20) & 48(5) & 44(4) & 51(4) & 11(3) & -32(4) & -9(3) \\ \mathrm{F}(21) & 83(6) & 53(4) & 60(5) & -19(4) & -47(4) & -3(4) \\ \mathrm{F}(1) & 27(1) & 32(1) & 29(1) & -10(1) & -10(1) & -5(1) \\ \mathrm{F}(2) & 26(1) & 25(1) & 25(1) & -4(1) & -6(1) & -7(1) \\ \mathrm{In}(1) & 33(1) & 32(1) & 30(1) & -4(1) & -13(1) & -9(1) \\ \mathrm{B}(1) & 32(8) & 12(5) & 24(6) & 2(4) & -16(5) & 0(5) \\ -------------------------------------------------------------- & \\ & & & & & \\ & & & & & \\ \end{array}$


Table 2.5 Hydrogen coordinates ( x 10 $)$ and isotropic displacement parameters $\left(\AA^{2} \times 10^{3}\right)$ for 8 .

\begin{tabular}{|c|c|c|c|c|}
\hline & $\mathrm{x}$ & $\mathrm{y}$ & $\mathrm{z}$ & $\mathrm{U}(\mathrm{eq})$ \\
\hline $\mathrm{H}(15 \mathrm{~A})$ & 14572 & 4932 & 6711 & 65 \\
\hline $\mathrm{H}(15 \mathrm{~B})$ & 15270 & 5701 & 5840 & 65 \\
\hline $\mathrm{H}(15 \mathrm{C})$ & 14139 & 5764 & 5882 & 65 \\
\hline $\mathrm{H}(16 \mathrm{~A})$ & 12038 & 7597 & 6542 & 61 \\
\hline $\mathrm{H}(16 \mathrm{~B})$ & 12051 & 6414 & 7138 & 61 \\
\hline $\mathrm{H}(16 \mathrm{C})$ & 12705 & 6789 & 6062 & 61 \\
\hline $\mathrm{H}(17 \mathrm{~A})$ & 12095 & 7680 & 8600 & 61 \\
\hline $\mathrm{H}(17 \mathrm{~B})$ & 11984 & 8382 & 7576 & 61 \\
\hline $\mathrm{H}(17 \mathrm{C})$ & 12681 & 8640 & 7999 & 61 \\
\hline $\mathrm{H}(18 \mathrm{~A})$ & 14337 & 6933 & 9041 & 72 \\
\hline $\mathrm{H}(18 \mathrm{~B})$ & 14167 & 8100 & 8408 & 72 \\
\hline $\mathrm{H}(18 \mathrm{C})$ & 15236 & 7448 & 8186 & 72 \\
\hline $\mathrm{H}(19 \mathrm{~A})$ & 16218 & 6331 & 7422 & 70 \\
\hline $\mathrm{H}(19 \mathrm{~B})$ & 16200 & 5713 & 6814 & 70 \\
\hline $\mathrm{H}(19 \mathrm{C})$ & 15755 & 5291 & 7934 & 70 \\
\hline $\mathrm{H}(20 \mathrm{~A})$ & 13253 & 7044 & 4401 & 125 \\
\hline $\mathrm{H}(20 \mathrm{~B})$ & 14334 & 7395 & 3969 & 125 \\
\hline $\mathrm{H}(20 \mathrm{C})$ & 13932 & 7010 & 3389 & 125 \\
\hline$H(21 A)$ & 11595 & 7601 & 4452 & 131 \\
\hline $\mathrm{H}(21 \mathrm{~B})$ & 11318 & 7905 & 3497 & 131 \\
\hline $\mathrm{H}(21 \mathrm{C})$ & 10825 & 8589 & 4142 & 131 \\
\hline $\mathrm{H}(22 \mathrm{~A})$ & 15237 & 8804 & 2901 & 159 \\
\hline $\mathrm{H}(22 \mathrm{~B})$ & 15077 & 9975 & 2279 & 159 \\
\hline $\mathrm{H}(22 \mathrm{C})$ & 15232 & 9132 & 1836 & 159 \\
\hline $\mathrm{H}(23 \mathrm{~A})$ & 13437 & 11093 & 994 & 129 \\
\hline $\mathrm{H}(23 \mathrm{~B})$ & 14031 & 11217 & 1594 & 129 \\
\hline $\mathrm{H}(23 \mathrm{C})$ & 12872 & 11643 & 1747 & 129 \\
\hline $\mathrm{H}(24 \mathrm{~A})$ & 10669 & 10090 & 3052 & 101 \\
\hline $\mathrm{H}(24 \mathrm{~B})$ & 11228 & 10393 & 1950 & 101 \\
\hline $\mathrm{H}(24 \mathrm{C})$ & 11193 & 11094 & 2503 & 101 \\
\hline $\mathrm{H}(32)$ & 8453 & 3697 & 6726 & 30 \\
\hline $\mathrm{H}(34)$ & 8712 & 6653 & 5235 & 36 \\
\hline $\mathrm{H}(36)$ & 9245 & 5345 & 7758 & 29 \\
\hline $\mathrm{H}(39)$ & 12887 & 1922 & 6859 & 29 \\
\hline $\mathrm{H}(41)$ & 10858 & 3796 & 8123 & 31 \\
\hline $\mathrm{H}(43)$ & 9932 & 1845 & 7514 & 31 \\
\hline $\mathrm{H}(44)$ & 7518 & 4525 & 9077 & 31 \\
\hline $\mathrm{H}(45)$ & 10030 & 2905 & 9677 & 30 \\
\hline $\mathrm{H}(48)$ & 8272 & 4667 & 11192 & 37 \\
\hline $\mathrm{H}(50)$ & 9159 & 1310 & 9326 & 35 \\
\hline $\mathrm{H}(51)$ & 7094 & 3433 & 8239 & 26 \\
\hline $\mathrm{H}(53)$ & 6475 & 591 & 9818 & 52 \\
\hline
\end{tabular}




\section{Details of crystal structure of compound $\mathbf{1 0}$}

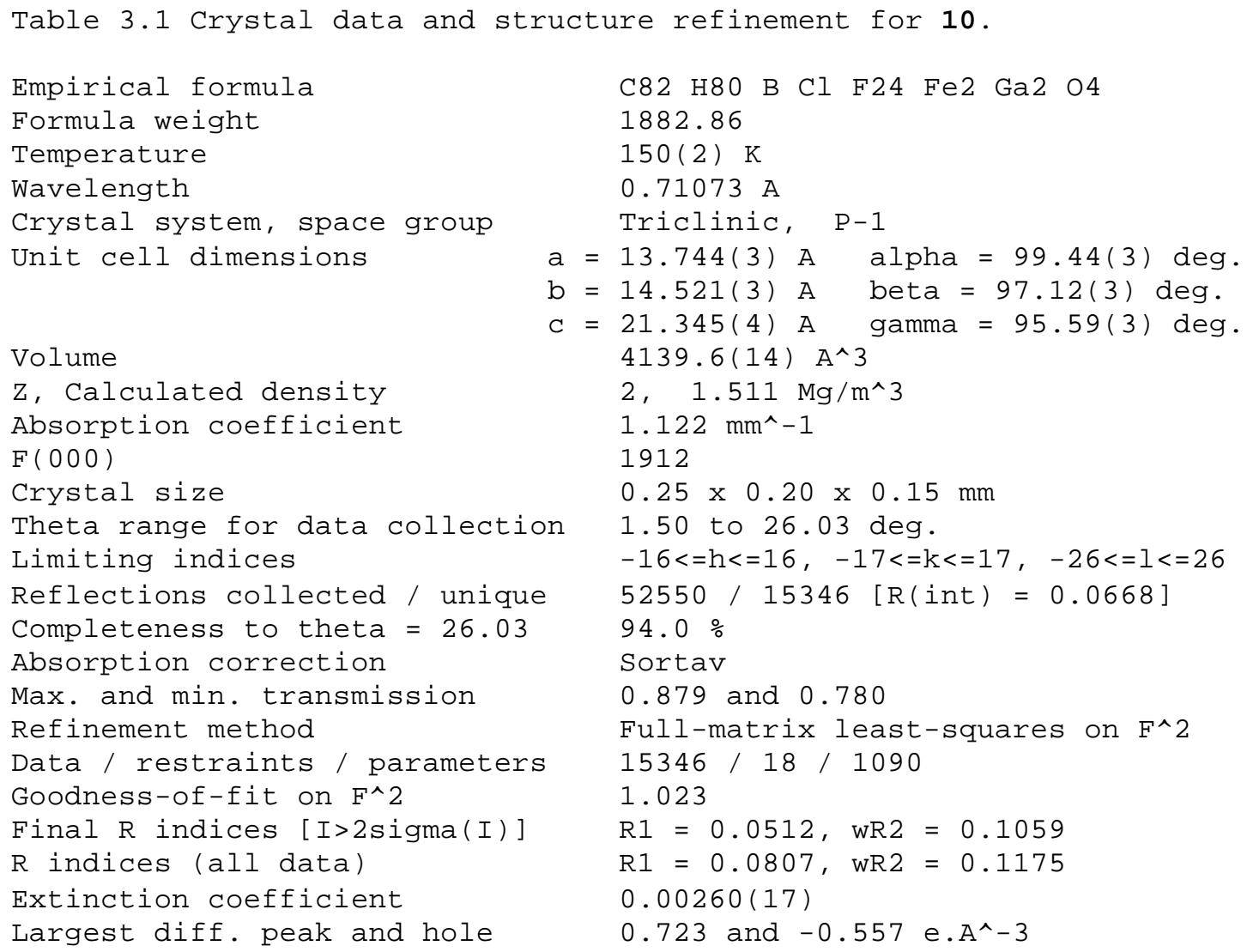


Table 2. Atomic coordinates ( $x$ 10^4) and equivalent isotropic displacement parameters $\left(A^{\wedge} 2 \times 10^{\wedge} 3\right)$ for 10 .

U(eq) is defined as one third of the trace of the orthogonalized Uij tensor.

\begin{tabular}{|c|c|c|c|c|}
\hline & $\mathrm{x}$ & $\mathrm{y}$ & $\mathrm{z}$ & $\mathrm{U}(\mathrm{eq})$ \\
\hline $\mathrm{Ga}(1)$ & $861(1)$ & $1645(1)$ & $2190(1)$ & $27(1)$ \\
\hline $\mathrm{Ga}(2)$ & $3509(1)$ & $-234(1)$ & $2204(1)$ & $29(1)$ \\
\hline $\operatorname{Fe}(1)$ & $-540(1)$ & $1274(1)$ & $1417(1)$ & $29(1)$ \\
\hline $\mathrm{Fe}(2)$ & $4770(1)$ & $-59(1)$ & $1583(1)$ & $32(1)$ \\
\hline $\mathrm{Cl}(1)$ & $2170(1)$ & $641(1)$ & $1809(1)$ & $24(1)$ \\
\hline$F(1)$ & $10137(2)$ & $-5653(2)$ & $1400(1)$ & $71(1)$ \\
\hline$F(2)$ & $10522(1)$ & $-4903(1)$ & $664(1)$ & $47(1)$ \\
\hline$F(3)$ & $9326(1)$ & $-6016(1)$ & $453(1)$ & $54(1)$ \\
\hline$F(4)$ & $8124(3)$ & $-2683(2)$ & $-43(1)$ & $86(1)$ \\
\hline$F(4 A)$ & $7335(7)$ & $-2437(7)$ & $327(5)$ & $53(3)$ \\
\hline$F(5)$ & $7221(2)$ & $-2156(2)$ & $610(2)$ & $81(1)$ \\
\hline$F(5 A)$ & $8174(8)$ & $-1630(7)$ & $1081(5)$ & $69(3)$ \\
\hline$F(6)$ & $8772(3)$ & $-1754(2)$ & $791(2)$ & $102(1)$ \\
\hline$F(6 A)$ & $8804(6)$ & $-2219(7)$ & $325(5)$ & $52(2)$ \\
\hline$F(7)$ & $10365(2)$ & $-4744(2)$ & $3477(1)$ & $95(1)$ \\
\hline$F(8)$ & $10171(2)$ & $-5851(2)$ & $4010(2)$ & $117(1)$ \\
\hline$F(9)$ & $9826(2)$ & $-4513(2)$ & $4346(1)$ & $75(1)$ \\
\hline$F(10)$ & $6486(3)$ & $-7947(2)$ & $3015(2)$ & $119(2)$ \\
\hline$F(10 A)$ & $5941(7)$ & $-7694(8)$ & $2958(5)$ & $27(3)$ \\
\hline$F(11)$ & $5642(2)$ & $-6998(2)$ & $3499(2)$ & $125(1)$ \\
\hline$F(11 A)$ & $6031(11)$ & $-7060(10)$ & $3863(7)$ & $59(4)$ \\
\hline$F(12)$ & $6854(3)$ & $-7457(2)$ & $4004(2)$ & $102(1)$ \\
\hline$F(12 A)$ & $7118(11)$ & $-7978(10)$ & $3505(8)$ & $67(4)$ \\
\hline$F(13)$ & $4260(1)$ & $-3913(1)$ & $3707(1)$ & $54(1)$ \\
\hline$F(14)$ & $3770(1)$ & $-2917(2)$ & $3151(1)$ & $70(1)$ \\
\hline$F(15)$ & $4459(1)$ & $-2452(2)$ & $4116(1)$ & $67(1)$ \\
\hline $\mathrm{F}(16)$ & $8108(2)$ & $-1164(1)$ & $4208(1)$ & $61(1)$ \\
\hline$F(17)$ & $7332(2)$ & $-402(1)$ & $3579(1)$ & $68(1)$ \\
\hline$F(18)$ & $8519(2)$ & $-1132(2)$ & $3288(1)$ & $65(1)$ \\
\hline $\mathrm{F}(19)$ & $3678(2)$ & $-3912(2)$ & $861(1)$ & $92(1)$ \\
\hline$F(20)$ & $2812(2)$ & $-5188(2)$ & $873(1)$ & $99(1)$ \\
\hline$F(21)$ & $3416(2)$ & $-4981(2)$ & $40(1)$ & $82(1)$ \\
\hline$F(22)$ & $4846(2)$ & $-8092(2)$ & $380(1)$ & $84(1)$ \\
\hline$F(23)$ & $6051(2)$ & $-7457(2)$ & $-4(1)$ & $97(1)$ \\
\hline$F(24)$ & $6291(2)$ & $-7890(1)$ & $903(1)$ & $63(1)$ \\
\hline$O(1)$ & $-1422(2)$ & $-67(2)$ & $2117(1)$ & $52(1)$ \\
\hline$O(2)$ & $-1324(2)$ & $2916(2)$ & $2048(1)$ & $64(1)$ \\
\hline$O(3)$ & $4751(2)$ & $1962(2)$ & $1851(2)$ & $72(1)$ \\
\hline$O(4)$ & $3330(2)$ & $-306(2)$ & $440(1)$ & $64(1)$ \\
\hline$C(1)$ & $-1052(2)$ & $482(2)$ & $1861(2)$ & $37(1)$ \\
\hline$C(2)$ & $-995(2)$ & $2269(2)$ & $1808(2)$ & $42(1)$ \\
\hline$C(3)$ & $425(2)$ & $1162(2)$ & $725(1)$ & $32(1)$ \\
\hline$C(4)$ & $-227(2)$ & $1816(2)$ & $605(2)$ & $39(1)$ \\
\hline$C(5)$ & $-1203(3)$ & $1342(2)$ & $487(2)$ & $43(1)$ \\
\hline$C(6)$ & $-1153(2)$ & $407(2)$ & $548(2)$ & $41(1)$ \\
\hline$C(7)$ & $-141(2)$ & $288(2)$ & $706(2)$ & $35(1)$ \\
\hline$C(8)$ & $1517(2)$ & $2369(2)$ & $3019(1)$ & $24(1)$ \\
\hline$C(9)$ & $1325(2)$ & $2068(2)$ & $3596(1)$ & $25(1)$ \\
\hline$C(10)$ & $1794(2)$ & $2585(2)$ & $4182(1)$ & $27(1)$ \\
\hline C (11) & $2449(2)$ & $3397(2)$ & $4226(2)$ & $28(1)$ \\
\hline $\mathrm{C}(12)$ & $2628(2)$ & $3676(2)$ & $3659(1)$ & $29(1)$ \\
\hline
\end{tabular}




\begin{tabular}{|c|c|c|c|c|}
\hline C (13) & $2182(2)$ & $3191(2)$ & $3059(1)$ & $27(1)$ \\
\hline C (14) & $552(2)$ & $1219(2)$ & $3600(1)$ & $26(1)$ \\
\hline$C(15)$ & $-484(2)$ & $1522(2)$ & $3447(2)$ & $33(1)$ \\
\hline$C(16)$ & $700(2)$ & $348(2)$ & $3126(1)$ & $29(1)$ \\
\hline C (17) & $614(2)$ & $904(2)$ & $4255(2)$ & $36(1)$ \\
\hline $\mathrm{C}(18)$ & $2967(2)$ & $3965(2)$ & $4868(2)$ & $35(1)$ \\
\hline$C(19)$ & $4075(3)$ & 3891 (3) & $4916(2)$ & $55(1)$ \\
\hline$C(20)$ & $2583(3)$ & $3619(3)$ & $5443(2)$ & $50(1)$ \\
\hline$C(21)$ & $2796(3)$ & $5006(2)$ & $4905(2)$ & $51(1)$ \\
\hline$C(22)$ & $2451(2)$ & $3638(2)$ & $2483(2)$ & $31(1)$ \\
\hline$C(23)$ & $3582(2)$ & $3697(3)$ & $2484(2)$ & $51(1)$ \\
\hline C (24) & $1952(3)$ & $3108(2)$ & $1831(2)$ & $40(1)$ \\
\hline$C(25)$ & $2143(3)$ & $4638(2)$ & $2548(2)$ & $43(1)$ \\
\hline $\mathrm{C}(26)$ & $4743(2)$ & $1161(3)$ & $1754(2)$ & $44(1)$ \\
\hline$C(27)$ & $3875(2)$ & $-208(3)$ & $902(2)$ & $43(1)$ \\
\hline$C(28)$ & $6295(2)$ & $47(3)$ & $1586(2)$ & $52(1)$ \\
\hline$C(29)$ & $5841(2)$ & $-679(3)$ & $1096(2)$ & $52(1)$ \\
\hline$C(30)$ & $5318(2)$ & $-1351(3)$ & $1380(2)$ & $51(1)$ \\
\hline$C(31)$ & $5468(2)$ & $-1039(2)$ & $2051(2)$ & $44(1)$ \\
\hline$C(32)$ & $6068(2)$ & $-160(2)$ & $2183(2)$ & $44(1)$ \\
\hline$C(33)$ & $2940(2)$ & $-786(2)$ & $2879(2)$ & $28(1)$ \\
\hline$C(34)$ & $2213(2)$ & $-1589(2)$ & $2716(2)$ & $28(1)$ \\
\hline$C(35)$ & $1788(2)$ & $-1919(2)$ & $3207(2)$ & $32(1)$ \\
\hline$C(36)$ & $2042(2)$ & $-1532(2)$ & $3850(2)$ & $30(1)$ \\
\hline$C(37)$ & $2761(2)$ & $-749(2)$ & $3998(2)$ & $30(1)$ \\
\hline $\mathrm{C}(38)$ & $3208(2)$ & $-366(2)$ & $3534(1)$ & $26(1)$ \\
\hline C (39) & $1817(2)$ & $-2118(2)$ & $2026(2)$ & $34(1)$ \\
\hline$C(40)$ & $1778(3)$ & $-3188(2)$ & $2002(2)$ & $53(1)$ \\
\hline$C(41)$ & $2447(3)$ & $-1887(2)$ & $1519(2)$ & $43(1)$ \\
\hline$C(42)$ & $767(2)$ & $-1879(3)$ & $1834(2)$ & $51(1)$ \\
\hline$C(43)$ & $1582(2)$ & $-1974(2)$ & $4362(2)$ & $34(1)$ \\
\hline$C(44)$ & $1987(2)$ & $-2913(2)$ & $4399(2)$ & $44(1)$ \\
\hline$C(45)$ & $450(2)$ & $-2142(2)$ & $4190(2)$ & $47(1)$ \\
\hline$C(46)$ & $1830(3)$ & $-1345(2)$ & $5027(2)$ & $47(1)$ \\
\hline$C(47)$ & $3992(2)$ & $506(2)$ & $3761(2)$ & $29(1)$ \\
\hline C ( 48$)$ & $5018(2)$ & $187(2)$ & $3716(2)$ & $38(1)$ \\
\hline$C(49)$ & $3984(2)$ & $966(2)$ & $4463(2)$ & $42(1)$ \\
\hline$C(50)$ & $3827(2)$ & $1268(2)$ & $3358(2)$ & $33(1)$ \\
\hline$C(51)$ & $7741(2)$ & $-4198(2)$ & $1727(2)$ & $29(1)$ \\
\hline$C(52)$ & $8466(2)$ & $-4769(2)$ & $1555(2)$ & $32(1)$ \\
\hline$C(53)$ & $9048(2)$ & $-4603(2)$ & $1089(1)$ & $30(1)$ \\
\hline$C(54)$ & $8939(2)$ & $-3858(2)$ & $772(2)$ & $31(1)$ \\
\hline$C(55)$ & $8228(2)$ & $-3273(2)$ & $933(2)$ & $29(1)$ \\
\hline$C(56)$ & $7653(2)$ & $-3441(2)$ & $1401(2)$ & $31(1)$ \\
\hline$C(57)$ & $9758(2)$ & $-5286(2)$ & $904(2)$ & $40(1)$ \\
\hline$C(58)$ & $8106(2)$ & $-2466(2)$ & $601(2)$ & $39(1)$ \\
\hline C (59) & $7396(2)$ & $-5139(2)$ & $2691(1)$ & $29(1)$ \\
\hline$C(60)$ & $8359(2)$ & $-4915(2)$ & $3027(2)$ & $32(1)$ \\
\hline$C(61)$ & $8739(2)$ & $-5423(2)$ & $3477(2)$ & $32(1)$ \\
\hline$C(62)$ & $8166(2)$ & $-6173(2)$ & $3625(2)$ & $37(1)$ \\
\hline$C(63)$ & $7203(2)$ & $-6401(2)$ & $3309(2)$ & $35(1)$ \\
\hline$C(64)$ & $6832(2)$ & $-5895(2)$ & $2860(1)$ & $31(1)$ \\
\hline$C(65)$ & $9762(2)$ & $-5160(3)$ & $3815(2)$ & $47(1)$ \\
\hline$C(66)$ & $6572(3)$ & $-7202(3)$ & $3459(2)$ & $55(1)$ \\
\hline$C(67)$ & $6607(2)$ & $-3601(2)$ & $2642(1)$ & $28(1)$ \\
\hline$C(68)$ & $5721(2)$ & $-3663(2)$ & $2895(1)$ & $29(1)$ \\
\hline$C(69)$ & $5481(2)$ & $-2949(2)$ & $3349(1)$ & $28(1)$ \\
\hline$C(70)$ & $6132(2)$ & $-2140(2)$ & $3572(1)$ & $29(1)$ \\
\hline$C(71)$ & $7020(2)$ & $-2057(2)$ & $3334(1)$ & $30(1)$ \\
\hline$C(72)$ & $7254(2)$ & $-2770(2)$ & $2878(1)$ & $29(1)$ \\
\hline$C(73)$ & $4503(2)$ & $-3052(2)$ & $3577(2)$ & $40(1)$ \\
\hline
\end{tabular}




\begin{tabular}{llrrr}
$C(74)$ & $7736(2)$ & $-1196(2)$ & $3596(2)$ & $36(1)$ \\
C (75) & $6047(2)$ & $-5109(2)$ & $1658(2)$ & $31(1)$ \\
C (76) & $5202(2)$ & $-4743(2)$ & $1410(2)$ & $33(1)$ \\
C $(77)$ & $4518(2)$ & $-5251(2)$ & $906(2)$ & $37(1)$ \\
C $(78)$ & $4650(2)$ & $-6150(2)$ & $631(2)$ & $43(1)$ \\
C $(79)$ & $5484(3)$ & $-6533(2)$ & $862(2)$ & $41(1)$ \\
C $(80)$ & $6157(2)$ & $-6020(2)$ & $1362(2)$ & $35(1)$ \\
C $(81)$ & $3615(3)$ & $-4840(3)$ & $673(2)$ & $47(1)$ \\
C $(82)$ & $5679(3)$ & $-7477(3)$ & $543(2)$ & $56(1)$ \\
B (1) & $6943(2)$ & $-4501(2)$ & $2187(2)$ & $29(1)$ \\
\hline
\end{tabular}




\begin{tabular}{|c|c|}
\hline $\mathrm{Ga}(1)-\mathrm{C}(8)$ & $1.964(3)$ \\
\hline $\mathrm{Ga}(1)-\mathrm{Fe}(1)$ & $2.3325(10)$ \\
\hline $\mathrm{Ga}(1)-\mathrm{Cl}(1)$ & $2.5516(10)$ \\
\hline $\mathrm{Ga}(2)-\mathrm{C}(33)$ & $1.967(3)$ \\
\hline $\mathrm{Ga}(2)-\mathrm{Fe}(2)$ & $2.3284(8)$ \\
\hline $\mathrm{Ga}(2)-\mathrm{Cl}(1)$ & $2.4759(10)$ \\
\hline $\mathrm{Fe}(1)-\mathrm{C}(1)$ & $1.755(4)$ \\
\hline $\mathrm{Fe}(1)-\mathrm{C}(2)$ & $1.757(3)$ \\
\hline $\mathrm{Fe}(1)-\mathrm{C}(7)$ & $2.074(3)$ \\
\hline $\mathrm{Fe}(1)-\mathrm{C}(4)$ & $2.091(3)$ \\
\hline $\mathrm{Fe}(1)-\mathrm{C}(6)$ & $2.092(3)$ \\
\hline $\mathrm{Fe}(1)-\mathrm{C}(5)$ & $2.102(3)$ \\
\hline $\mathrm{Fe}(1)-\mathrm{C}(3)$ & $2.102(3)$ \\
\hline $\mathrm{Fe}(2)-\mathrm{C}(27)$ & $1.754(3)$ \\
\hline $\mathrm{Fe}(2)-\mathrm{C}(26)$ & $1.754(4)$ \\
\hline $\mathrm{Fe}(2)-\mathrm{C}(28)$ & $2.085(3)$ \\
\hline $\mathrm{Fe}(2)-\mathrm{C}(30)$ & $2.089(3)$ \\
\hline $\mathrm{Fe}(2)-\mathrm{C}(29)$ & $2.095(4)$ \\
\hline $\mathrm{Fe}(2)-\mathrm{C}(32)$ & $2.095(3)$ \\
\hline $\mathrm{Fe}(2)-\mathrm{C}(31)$ & $2.106(4)$ \\
\hline$F(1)-C(57)$ & $1.334(4)$ \\
\hline$F(2)-C(57)$ & $1.337(4)$ \\
\hline$F(3)-C(57)$ & $1.342(4)$ \\
\hline$F(4)-C(58)$ & $1.364(4)$ \\
\hline$F(4 A)-C(58)$ & $1.153(10)$ \\
\hline$F(5)-C(58)$ & $1.339(4)$ \\
\hline$F(5 A)-C(58)$ & $1.442(11)$ \\
\hline$F(6)-C(58)$ & $1.288(4)$ \\
\hline$F(6 A)-C(58)$ & $1.241(10)$ \\
\hline$F(7)-C(65)$ & $1.329(4)$ \\
\hline$F(8)-C(65)$ & $1.297(4)$ \\
\hline$F(9)-C(65)$ & $1.335(4)$ \\
\hline$F(10)-C(66)$ & $1.302(5)$ \\
\hline$F(10 A)-C(66)$ & $1.344(11)$ \\
\hline$F(11)-C(66)$ & $1.350(5)$ \\
\hline$F(11 A)-C(66)$ & $1.213(15)$ \\
\hline$F(12)-C(66)$ & $1.303(5)$ \\
\hline$F(12 A)-C(66)$ & $1.423(15)$ \\
\hline$F(13)-C(73)$ & $1.344(4)$ \\
\hline$F(14)-C(73)$ & $1.324(4)$ \\
\hline$F(15)-C(73)$ & $1.336(4)$ \\
\hline$F(16)-C(74)$ & $1.335(4)$ \\
\hline$F(17)-C(74)$ & $1.330(4)$ \\
\hline$F(18)-C(74)$ & $1.332(4)$ \\
\hline$F(19)-C(81)$ & $1.334(4)$ \\
\hline$F(20)-C(81)$ & $1.317(4)$ \\
\hline$F(21)-C(81)$ & $1.322(4)$ \\
\hline$F(22)-C(82)$ & $1.353(4)$ \\
\hline$F(23)-C(82)$ & $1.334(5)$ \\
\hline$F(24)-C(82)$ & $1.322(5)$ \\
\hline $\mathrm{O}(1)-\mathrm{C}(1)$ & $1.150(4)$ \\
\hline $\mathrm{O}(2)-\mathrm{C}(2)$ & $1.154(4)$ \\
\hline $\mathrm{O}(3)-\mathrm{C}(26)$ & $1.147(4)$ \\
\hline $\mathrm{O}(4)-\mathrm{C}(27)$ & $1.141(4)$ \\
\hline$C(3)-C(4)$ & $1.400(5)$ \\
\hline$C(3)-C(7)$ & $1.415(4)$ \\
\hline$C(4)-C(5)$ & $1.420(5)$ \\
\hline$C(5)-C(6)$ & $1.392(5)$ \\
\hline
\end{tabular}




\begin{tabular}{|c|c|}
\hline$C(6)-C(7)$ & $1.424(5)$ \\
\hline$C(8)-C(13)$ & $1.415(4)$ \\
\hline$C(8)-C(9)$ & $1.419(4)$ \\
\hline$C(9)-C(10)$ & $1.393(4)$ \\
\hline$C(9)-C(14)$ & $1.549(4)$ \\
\hline$C(10)-C(11)$ & $1.394(4)$ \\
\hline$C(11)-C(12)$ & $1.381(4)$ \\
\hline$C(11)-C(18)$ & $1.530(4)$ \\
\hline$C(12)-C(13)$ & $1.392(4)$ \\
\hline$C(13)-C(22)$ & $1.548(4)$ \\
\hline$C(14)-C(16)$ & $1.532(4)$ \\
\hline$C(14)-C(17)$ & $1.535(4)$ \\
\hline$C(14)-C(15)$ & $1.544(4)$ \\
\hline$C(18)-C(19)$ & $1.529(5)$ \\
\hline$C(18)-C(20)$ & $1.532(5)$ \\
\hline$C(18)-C(21)$ & $1.543(5)$ \\
\hline$C(22)-C(24)$ & $1.519(4)$ \\
\hline$C(22)-C(25)$ & $1.541(4)$ \\
\hline$C(22)-C(23)$ & $1.548(4)$ \\
\hline$C(28)-C(29)$ & $1.394(5)$ \\
\hline$C(28)-C(32)$ & $1.420(5)$ \\
\hline$C(29)-C(30)$ & $1.416(5)$ \\
\hline$C(30)-C(31)$ & $1.411(5)$ \\
\hline$C(31)-C(32)$ & $1.417(5)$ \\
\hline$C(33)-C(38)$ & $1.418(4)$ \\
\hline$C(33)-C(34)$ & $1.426(4)$ \\
\hline$C(34)-C(35)$ & $1.391(4)$ \\
\hline$C(34)-C(39)$ & $1.549(4)$ \\
\hline$C(35)-C(36)$ & $1.381(4)$ \\
\hline$C(36)-C(37)$ & $1.398(4)$ \\
\hline$C(36)-C(43)$ & $1.527(4)$ \\
\hline$C(37)-C(38)$ & $1.391(4)$ \\
\hline$C(38)-C(47)$ & $1.550(4)$ \\
\hline$C(39)-C(41)$ & $1.526(5)$ \\
\hline$C(39)-C(42)$ & $1.540(4)$ \\
\hline$C(39)-C(40)$ & $1.543(4)$ \\
\hline$C(43)-C(44)$ & $1.532(4)$ \\
\hline$C(43)-C(46)$ & $1.535(5)$ \\
\hline$C(43)-C(45)$ & $1.540(4)$ \\
\hline$C(47)-C(50)$ & $1.525(4)$ \\
\hline$C(47)-C(48)$ & $1.536(4)$ \\
\hline$C(47)-C(49)$ & $1.539(4)$ \\
\hline$C(51)-C(56)$ & $1.401(4)$ \\
\hline$C(51)-C(52)$ & $1.403(4)$ \\
\hline $\mathrm{C}(51)-\mathrm{B}(1)$ & $1.636(5)$ \\
\hline$C(52)-C(53)$ & $1.386(4)$ \\
\hline$C(53)-C(54)$ & $1.378(4)$ \\
\hline$C(53)-C(57)$ & $1.499(4)$ \\
\hline$C(54)-C(55)$ & $1.395(4)$ \\
\hline$C(55)-C(56)$ & $1.387(4)$ \\
\hline$C(55)-C(58)$ & $1.477(5)$ \\
\hline$C(59)-C(64)$ & $1.404(4)$ \\
\hline$C(59)-C(60)$ & $1.405(4)$ \\
\hline$C(59)-B(1)$ & $1.635(5)$ \\
\hline$C(60)-C(61)$ & $1.387(4)$ \\
\hline$C(61)-C(62)$ & $1.383(4)$ \\
\hline$C(61)-C(65)$ & $1.482(4)$ \\
\hline$C(62)-C(63)$ & $1.391(4)$ \\
\hline$C(63)-C(64)$ & $1.380(4)$ \\
\hline$C(63)-C(66)$ & $1.483(5)$ \\
\hline$C(67)-C(68)$ & $1.392(4)$ \\
\hline
\end{tabular}




\begin{tabular}{|c|c|}
\hline$C(67)-C(72)$ & $1.407(4)$ \\
\hline $\mathrm{C}(67)-\mathrm{B}(1)$ & $1.637(4)$ \\
\hline$C(68)-C(69)$ & $1.394(4)$ \\
\hline$C(69)-C(70)$ & $1.383(4)$ \\
\hline$C(69)-C(73)$ & $1.487(4)$ \\
\hline$C(70)-C(71)$ & $1.382(4)$ \\
\hline$C(71)-C(72)$ & $1.390(4)$ \\
\hline$C(71)-C(74)$ & $1.496(4)$ \\
\hline$C(75)-C(80)$ & $1.400(4)$ \\
\hline$C(75)-C(76)$ & $1.405(4)$ \\
\hline $\mathrm{C}(75)-\mathrm{B}(1)$ & $1.639(4)$ \\
\hline$C(76)-C(77)$ & $1.391(4)$ \\
\hline$C(77)-C(78)$ & $1.380(5)$ \\
\hline$C(77)-C(81)$ & $1.494(5)$ \\
\hline$C(78)-C(79)$ & $1.393(5)$ \\
\hline$C(79)-C(80)$ & $1.383(4)$ \\
\hline$C(79)-C(82)$ & $1.491(5)$ \\
\hline $\mathrm{C}(8)-\mathrm{Ga}(1)-\mathrm{Fe}(1)$ & $149.07(8)$ \\
\hline $\mathrm{C}(8)-\mathrm{Ga}(1)-\mathrm{Cl}(1)$ & $103.24(8)$ \\
\hline $\mathrm{Fe}(1)-\mathrm{Ga}(1)-\mathrm{Cl}(1)$ & $107.69(3)$ \\
\hline $\mathrm{C}(33)-\mathrm{Ga}(2)-\mathrm{Fe}(2)$ & $150.50(9)$ \\
\hline $\mathrm{C}(33)-\mathrm{Ga}(2)-\mathrm{Cl}(1)$ & $101.95(9)$ \\
\hline $\mathrm{Fe}(2)-\mathrm{Ga}(2)-\mathrm{Cl}(1)$ & $107.46(3)$ \\
\hline$C(1)-F e(1)-C(2)$ & $96.04(16)$ \\
\hline $\mathrm{C}(1)-\mathrm{Fe}(1)-\mathrm{C}(7)$ & $97.48(14)$ \\
\hline$C(2)-F e(1)-C(7)$ & $161.86(15)$ \\
\hline $\mathrm{C}(1)-\mathrm{Fe}(1)-\mathrm{C}(4)$ & $157.78(13)$ \\
\hline $\mathrm{C}(2)-\mathrm{Fe}(1)-\mathrm{C}(4)$ & $97.28(15)$ \\
\hline$C(7)-F e(1)-C(4)$ & $66.28(13)$ \\
\hline $\mathrm{C}(1)-\mathrm{Fe}(1)-\mathrm{C}(6)$ & $91.81(14)$ \\
\hline$C(2)-F e(1)-C(6)$ & $127.63(15)$ \\
\hline$C(7)-\mathrm{Fe}(1)-\mathrm{C}(6)$ & $39.99(13)$ \\
\hline $\mathrm{C}(4)-\mathrm{Fe}(1)-\mathrm{C}(6)$ & $65.98(13)$ \\
\hline $\mathrm{C}(1)-\mathrm{Fe}(1)-\mathrm{C}(5)$ & $121.09(14)$ \\
\hline$C(2)-F e(1)-C(5)$ & $96.40(15)$ \\
\hline$C(7)-\mathrm{Fe}(1)-\mathrm{C}(5)$ & $66.18(13)$ \\
\hline $\mathrm{C}(4)-\mathrm{Fe}(1)-\mathrm{C}(5)$ & $39.59(13)$ \\
\hline$C(6)-F e(1)-C(5)$ & $38.78(13)$ \\
\hline $\mathrm{C}(1)-\mathrm{Fe}(1)-\mathrm{C}(3)$ & $133.66(14)$ \\
\hline$C(2)-F e(1)-C(3)$ & $129.98(15)$ \\
\hline$C(7)-F e(1)-C(3)$ & $39.59(12)$ \\
\hline $\mathrm{C}(4)-\mathrm{Fe}(1)-\mathrm{C}(3)$ & $38.99(12)$ \\
\hline$C(6)-F e(1)-C(3)$ & $66.19(13)$ \\
\hline$C(5)-F e(1)-C(3)$ & $65.79(13)$ \\
\hline $\mathrm{C}(1)-\mathrm{Fe}(1)-\mathrm{Ga}(1)$ & $90.19(10)$ \\
\hline $\mathrm{C}(2)-\mathrm{Fe}(1)-\mathrm{Ga}(1)$ & $87.51(11)$ \\
\hline $\mathrm{C}(7)-\mathrm{Fe}(1)-\mathrm{Ga}(1)$ & $104.45(9)$ \\
\hline $\mathrm{C}(4)-\mathrm{Fe}(1)-\mathrm{Ga}(1)$ & $108.07(9)$ \\
\hline$C(6)-F e(1)-G a(1)$ & $144.29(10)$ \\
\hline $\mathrm{C}(5)-\mathrm{Fe}(1)-\mathrm{Ga}(1)$ & $147.64(10)$ \\
\hline $\mathrm{C}(3)-\mathrm{Fe}(1)-\mathrm{Ga}(1)$ & $87.14(8)$ \\
\hline$C(27)-\mathrm{Fe}(2)-\mathrm{C}(26)$ & $93.21(17)$ \\
\hline$C(27)-\mathrm{Fe}(2)-\mathrm{C}(28)$ & $125.87(16)$ \\
\hline$C(26)-\mathrm{Fe}(2)-\mathrm{C}(28)$ & $94.25(16)$ \\
\hline$C(27)-\operatorname{Fe}(2)-C(30)$ & $97.73(16)$ \\
\hline$C(26)-\mathrm{Fe}(2)-\mathrm{C}(30)$ & $160.06(15)$ \\
\hline$C(28)-F e(2)-C(30)$ & $65.84(15)$ \\
\hline$C(27)-\operatorname{Fe}(2)-C(29)$ & $95.08(15)$ \\
\hline$C(26)-\mathrm{Fe}(2)-\mathrm{C}(29)$ & $123.01(16)$ \\
\hline$C(28)-F e(2)-C(29)$ & $38.97(14)$ \\
\hline
\end{tabular}




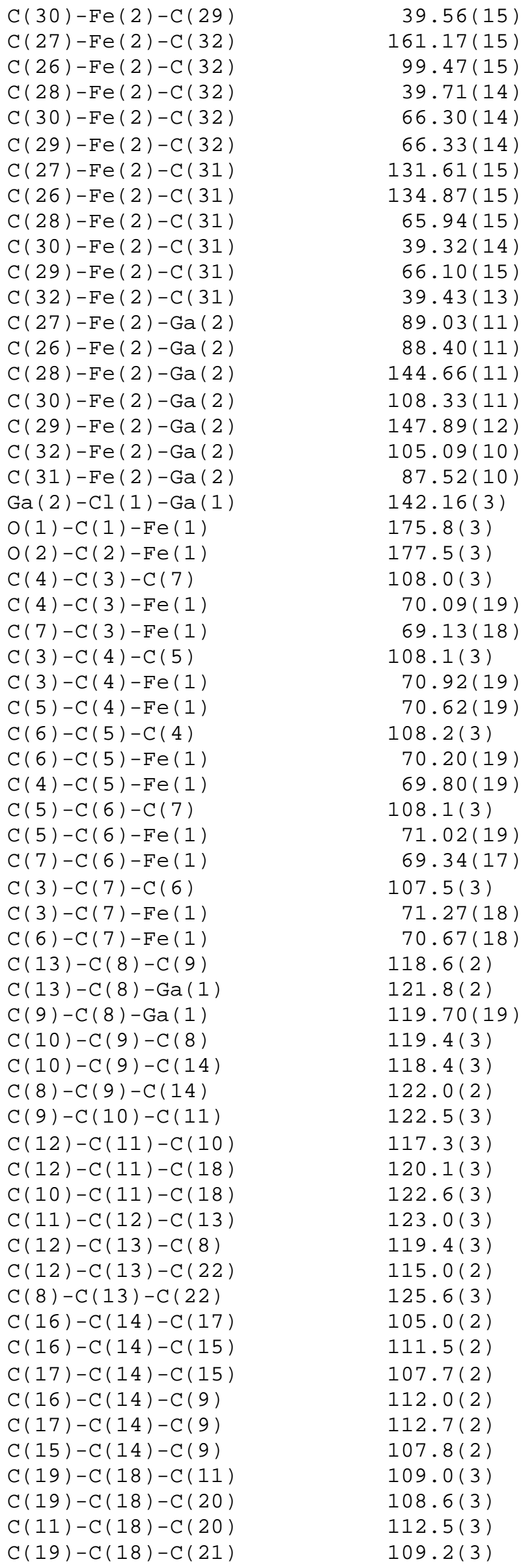




\begin{tabular}{|c|c|}
\hline$C(11)-C(18)-C(21)$ & $109.4(3)$ \\
\hline$C(20)-C(18)-C(21)$ & $108.0(3)$ \\
\hline$C(24)-C(22)-C(25)$ & $106.6(3)$ \\
\hline$C(24)-C(22)-C(13)$ & $114.6(2)$ \\
\hline$C(25)-C(22)-C(13)$ & $109.5(2)$ \\
\hline$C(24)-C(22)-C(23)$ & $108.0(3)$ \\
\hline$C(25)-C(22)-C(23)$ & $109.1(3)$ \\
\hline$C(13)-C(22)-C(23)$ & $109.0(3)$ \\
\hline$O(3)-C(26)-\operatorname{Fe}(2)$ & $177.4(3)$ \\
\hline$O(4)-C(27)-\mathrm{Fe}(2)$ & $176.6(3)$ \\
\hline$C(29)-C(28)-C(32)$ & $109.1(3)$ \\
\hline$C(29)-C(28)-\operatorname{Fe}(2)$ & $70.9(2)$ \\
\hline$C(32)-C(28)-\operatorname{Fe}(2)$ & $70.53(19)$ \\
\hline$C(28)-C(29)-C(30)$ & $107.7(3)$ \\
\hline$C(28)-C(29)-\operatorname{Fe}(2)$ & $70.1(2)$ \\
\hline$C(30)-C(29)-\operatorname{Fe}(2)$ & $70.0(2)$ \\
\hline$C(31)-C(30)-C(29)$ & $108.2(3)$ \\
\hline$C(31)-C(30)-\operatorname{Fe}(2)$ & $71.0(2)$ \\
\hline$C(29)-C(30)-\operatorname{Fe}(2)$ & $70.4(2)$ \\
\hline$C(30)-C(31)-C(32)$ & $108.0(3)$ \\
\hline$C(30)-C(31)-\operatorname{Fe}(2)$ & $69.7(2)$ \\
\hline$C(32)-C(31)-\operatorname{Fe}(2)$ & $69.9(2)$ \\
\hline$C(31)-C(32)-C(28)$ & $107.0(3)$ \\
\hline$C(31)-C(32)-\operatorname{Fe}(2)$ & $70.67(19)$ \\
\hline$C(28)-C(32)-F e(2)$ & $69.75(19)$ \\
\hline$C(38)-C(33)-C(34)$ & $118.9(3)$ \\
\hline $\mathrm{C}(38)-\mathrm{C}(33)-\mathrm{Ga}(2)$ & $120.6(2)$ \\
\hline $\mathrm{C}(34)-\mathrm{C}(33)-\mathrm{Ga}(2)$ & $120.4(2)$ \\
\hline$C(35)-C(34)-C(33)$ & $118.3(3)$ \\
\hline$C(35)-C(34)-C(39)$ & $116.0(3)$ \\
\hline$C(33)-C(34)-C(39)$ & $125.6(3)$ \\
\hline$C(36)-C(35)-C(34)$ & $124.1(3)$ \\
\hline$C(35)-C(36)-C(37)$ & $116.5(3)$ \\
\hline$C(35)-C(36)-C(43)$ & $120.7(3)$ \\
\hline$C(37)-C(36)-C(43)$ & $122.8(3)$ \\
\hline$C(38)-C(37)-C(36)$ & $122.9(3)$ \\
\hline$C(37)-C(38)-C(33)$ & $119.3(3)$ \\
\hline$C(37)-C(38)-C(47)$ & $118.0(3)$ \\
\hline$C(33)-C(38)-C(47)$ & $122.8(3)$ \\
\hline$C(41)-C(39)-C(42)$ & $108.3(3)$ \\
\hline$C(41)-C(39)-C(40)$ & $106.2(3)$ \\
\hline$C(42)-C(39)-C(40)$ & $108.9(3)$ \\
\hline$C(41)-C(39)-C(34)$ & $114.4(2)$ \\
\hline$C(42)-C(39)-C(34)$ & $108.9(3)$ \\
\hline$C(40)-C(39)-C(34)$ & $110.0(3)$ \\
\hline$C(36)-C(43)-C(44)$ & $108.5(3)$ \\
\hline$C(36)-C(43)-C(46)$ & $112.0(2)$ \\
\hline$C(44)-C(43)-C(46)$ & $108.5(3)$ \\
\hline$C(36)-C(43)-C(45)$ & $110.3(3)$ \\
\hline$C(44)-C(43)-C(45)$ & $109.6(3)$ \\
\hline$C(46)-C(43)-C(45)$ & $107.9(3)$ \\
\hline$C(50)-C(47)-C(48)$ & $110.3(3)$ \\
\hline$C(50)-C(47)-C(49)$ & $106.6(2)$ \\
\hline$C(48)-C(47)-C(49)$ & $107.2(3)$ \\
\hline$C(50)-C(47)-C(38)$ & $111.6(2)$ \\
\hline$C(48)-C(47)-C(38)$ & $108.2(2)$ \\
\hline$C(49)-C(47)-C(38)$ & $112.8(3)$ \\
\hline$C(56)-C(51)-C(52)$ & $115.3(3)$ \\
\hline$C(56)-C(51)-B(1)$ & $122.4(3)$ \\
\hline$C(52)-C(51)-B(1)$ & $121.5(3)$ \\
\hline$C(53)-C(52)-C(51)$ & $122.4(3)$ \\
\hline
\end{tabular}




\begin{tabular}{|c|c|}
\hline$C(54)-C(53)-C(52)$ & $121.1(3)$ \\
\hline$C(54)-C(53)-C(57)$ & $120.0(3)$ \\
\hline$C(52)-C(53)-C(57)$ & $118.8(3)$ \\
\hline$C(53)-C(54)-C(55)$ & $118.1(3)$ \\
\hline$C(56)-C(55)-C(54)$ & $120.4(3)$ \\
\hline$C(56)-C(55)-C(58)$ & $120.7(3)$ \\
\hline$C(54)-C(55)-C(58)$ & $118.9(3)$ \\
\hline$C(55)-C(56)-C(51)$ & $122.7(3)$ \\
\hline$F(1)-C(57)-F(2)$ & $106.6(3)$ \\
\hline$F(1)-C(57)-F(3)$ & $106.2(3)$ \\
\hline$F(2)-C(57)-F(3)$ & $105.9(3)$ \\
\hline$F(1)-C(57)-C(53)$ & $112.1(3)$ \\
\hline$F(2)-C(57)-C(53)$ & $113.3(3)$ \\
\hline$F(3)-C(57)-C(53)$ & $112.2(3)$ \\
\hline$F(4 A)-C(58)-F(6 A)$ & $114.5(7)$ \\
\hline$F(4 A)-C(58)-F(6)$ & $124.6(6)$ \\
\hline$F(6 A)-C(58)-F(6)$ & $52.3(5)$ \\
\hline$F(4 A)-C(58)-F(5)$ & $32.2(5)$ \\
\hline$F(6 A)-C(58)-F(5)$ & $129.8(5)$ \\
\hline$F(6)-C(58)-F(5)$ & $108.0(3)$ \\
\hline$F(4 A)-C(58)-F(4)$ & $70.2(6)$ \\
\hline$F(6 A)-C(58)-F(4)$ & $55.2(5)$ \\
\hline$F(6)-C(58)-F(4)$ & $104.1(3)$ \\
\hline$F(5)-C(58)-F(4)$ & $100.7(3)$ \\
\hline$F(4 A)-C(58)-F(5 A)$ & $99.4(7)$ \\
\hline$F(6 A)-C(58)-F(5 A)$ & $98.1(6)$ \\
\hline$F(6)-C(58)-F(5 A)$ & $47.0(4)$ \\
\hline$F(5)-C(58)-F(5 A)$ & $69.8(5)$ \\
\hline$F(4)-C(58)-F(5 A)$ & $137.5(5)$ \\
\hline$F(4 A)-C(58)-C(55)$ & $117.1(6)$ \\
\hline$F(6 A)-C(58)-C(55)$ & $115.9(5)$ \\
\hline$F(6)-C(58)-C(55)$ & $115.3(3)$ \\
\hline$F(5)-C(58)-C(55)$ & $114.1(3)$ \\
\hline$F(4)-C(58)-C(55)$ & $113.2(3)$ \\
\hline$F(5 A)-C(58)-C(55)$ & $108.2(5)$ \\
\hline$C(64)-C(59)-C(60)$ & $115.2(3)$ \\
\hline$C(64)-C(59)-B(1)$ & $122.7(3)$ \\
\hline$C(60)-C(59)-B(1)$ & $121.9(3)$ \\
\hline$C(61)-C(60)-C(59)$ & $122.7(3)$ \\
\hline$C(62)-C(61)-C(60)$ & $120.5(3)$ \\
\hline$C(62)-C(61)-C(65)$ & $119.0(3)$ \\
\hline$C(60)-C(61)-C(65)$ & $120.5(3)$ \\
\hline$C(61)-C(62)-C(63)$ & $118.2(3)$ \\
\hline$C(64)-C(63)-C(62)$ & $120.8(3)$ \\
\hline$C(64)-C(63)-C(66)$ & $119.9(3)$ \\
\hline$C(62)-C(63)-C(66)$ & $119.3(3)$ \\
\hline$C(63)-C(64)-C(59)$ & $122.5(3)$ \\
\hline$F(8)-C(65)-F(7)$ & $108.4(3)$ \\
\hline$F(8)-C(65)-F(9)$ & $104.3(3)$ \\
\hline$F(7)-C(65)-F(9)$ & $102.0(3)$ \\
\hline$F(8)-C(65)-C(61)$ & $114.3(3)$ \\
\hline$F(7)-C(65)-C(61)$ & $113.6(3)$ \\
\hline$F(9)-C(65)-C(61)$ & $113.2(3)$ \\
\hline$F(11 A)-C(66)-F(10)$ & $125.6(8)$ \\
\hline$F(11 A)-C(66)-F(12)$ & $64.4(8)$ \\
\hline$F(10)-C(66)-F(12)$ & $107.1(4)$ \\
\hline$F(11 A)-C(66)-F(10 A)$ & $101.6(9)$ \\
\hline$F(10)-C(66)-F(10 A)$ & $38.4(5)$ \\
\hline$F(12)-C(66)-F(10 A)$ & $127.8(6)$ \\
\hline$F(11 A)-C(66)-F(11)$ & $41.1(7)$ \\
\hline$F(10)-C(66)-F(11)$ & $105.5(4)$ \\
\hline
\end{tabular}




\begin{tabular}{|c|c|}
\hline$F(12)-C(66)-F(11)$ & $104.3(4)$ \\
\hline$F(10 A)-C(66)-F(11)$ & $69.2(5)$ \\
\hline$F(11 A)-C(66)-F(12 A)$ & $114.6(10)$ \\
\hline$F(10)-C(66)-F(12 A)$ & $56.0(6)$ \\
\hline$F(12)-C(66)-F(12 A)$ & $57.4(7)$ \\
\hline$F(10 A)-C(66)-F(12 A)$ & $92.1(8)$ \\
\hline$F(11)-C(66)-F(12 A)$ & $138.0(7)$ \\
\hline$F(11 A)-C(66)-C(63)$ & $119.7(8)$ \\
\hline$F(10)-C(66)-C(63)$ & $112.3(4)$ \\
\hline$F(12)-C(66)-C(63)$ & $115.4(3)$ \\
\hline$F(10 A)-C(66)-C(63)$ & $114.8(6)$ \\
\hline$F(11)-C(66)-C(63)$ & $111.4(3)$ \\
\hline$F(12 A)-C(66)-C(63)$ & $110.5(7)$ \\
\hline$C(68)-C(67)-C(72)$ & $115.8(3)$ \\
\hline$C(68)-C(67)-B(1)$ & $121.6(2)$ \\
\hline$C(72)-C(67)-B(1)$ & $121.9(3)$ \\
\hline$C(67)-C(68)-C(69)$ & $122.5(3)$ \\
\hline$C(70)-C(69)-C(68)$ & $120.4(3)$ \\
\hline$C(70)-C(69)-C(73)$ & $120.3(3)$ \\
\hline$C(68)-C(69)-C(73)$ & $119.3(3)$ \\
\hline$C(71)-C(70)-C(69)$ & $118.6(3)$ \\
\hline$C(70)-C(71)-C(72)$ & $120.7(3)$ \\
\hline$C(70)-C(71)-C(74)$ & $118.2(3)$ \\
\hline$C(72)-C(71)-C(74)$ & $121.0(3)$ \\
\hline$C(71)-C(72)-C(67)$ & $121.9(3)$ \\
\hline$F(14)-C(73)-F(15)$ & $106.4(3)$ \\
\hline$F(14)-C(73)-F(13)$ & $105.5(3)$ \\
\hline$F(15)-C(73)-F(13)$ & $105.6(3)$ \\
\hline$F(14)-C(73)-C(69)$ & $112.8(3)$ \\
\hline$F(15)-C(73)-C(69)$ & $113.1(3)$ \\
\hline$F(13)-C(73)-C(69)$ & $112.8(3)$ \\
\hline$F(17)-C(74)-F(18)$ & $106.4(3)$ \\
\hline$F(17)-C(74)-F(16)$ & $105.9(3)$ \\
\hline$F(18)-C(74)-F(16)$ & $105.0(3)$ \\
\hline $\mathrm{F}(17)-\mathrm{C}(74)-\mathrm{C}(71)$ & $113.0(3)$ \\
\hline$F(18)-C(74)-C(71)$ & $113.6(3)$ \\
\hline $\mathrm{F}(16)-\mathrm{C}(74)-\mathrm{C}(71)$ & $112.3(3)$ \\
\hline$C(80)-C(75)-C(76)$ & $115.3(3)$ \\
\hline$C(80)-C(75)-B(1)$ & $119.7(3)$ \\
\hline$C(76)-C(75)-B(1)$ & $124.5(3)$ \\
\hline$C(77)-C(76)-C(75)$ & $122.4(3)$ \\
\hline$C(78)-C(77)-C(76)$ & $120.4(3)$ \\
\hline$C(78)-C(77)-C(81)$ & $119.2(3)$ \\
\hline$C(76)-C(77)-C(81)$ & $120.3(3)$ \\
\hline$C(77)-C(78)-C(79)$ & $118.8(3)$ \\
\hline$C(80)-C(79)-C(78)$ & $120.1(3)$ \\
\hline$C(80)-C(79)-C(82)$ & $120.0(3)$ \\
\hline$C(78)-C(79)-C(82)$ & $119.8(3)$ \\
\hline$C(79)-C(80)-C(75)$ & $122.9(3)$ \\
\hline$F(20)-C(81)-F(21)$ & $105.8(3)$ \\
\hline$F(20)-C(81)-F(19)$ & $105.4(3)$ \\
\hline$F(21)-C(81)-F(19)$ & $105.3(3)$ \\
\hline$F(20)-C(81)-C(77)$ & $113.2(3)$ \\
\hline$F(21)-C(81)-C(77)$ & $113.1(3)$ \\
\hline$F(19)-C(81)-C(77)$ & $113.4(3)$ \\
\hline$F(24)-C(82)-F(23)$ & $106.6(4)$ \\
\hline$F(24)-C(82)-F(22)$ & $105.0(3)$ \\
\hline$F(23)-C(82)-F(22)$ & $105.2(3)$ \\
\hline$F(24)-C(82)-C(79)$ & $114.0(3)$ \\
\hline$F(23)-C(82)-C(79)$ & $113.1(3)$ \\
\hline$F(22)-C(82)-C(79)$ & $112.2(3)$ \\
\hline
\end{tabular}




$\begin{array}{ll}C(59)-B(1)-C(51) & 112.1(2) \\ C(59)-B(1)-C(67) & 104.5(2) \\ C(51)-B(1)-C(67) & 113.1(2) \\ C(59)-B(1)-C(75) & 111.6(2) \\ C(51)-B(1)-C(75) & 101.6(2) \\ C(67)-B(1)-C(75) & 114.2(2)\end{array}$

Symmetry transformations used to generate equivalent atoms: 
Table 3.4 Anisotropic displacement parameters (A^2 x 10^3) for 10.

The anisotropic displacement factor exponent takes the form:

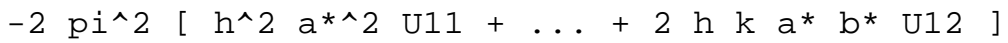

\begin{tabular}{|c|c|c|c|c|c|c|}
\hline & $\mathrm{U} 11$ & U22 & U33 & $\mathrm{U} 23$ & U13 & $\mathrm{U} 12$ \\
\hline $\mathrm{Ga}(1)$ & $28(1)$ & $25(1)$ & $27(1)$ & $3(1)$ & $3(1)$ & $3(1)$ \\
\hline $\mathrm{Ga}(2)$ & $28(1)$ & $27(1)$ & $31(1)$ & $4(1)$ & $3(1)$ & $5(1)$ \\
\hline $\mathrm{Fe}(1)$ & $30(1)$ & $28(1)$ & $28(1)$ & $3(1)$ & $2(1)$ & $7(1)$ \\
\hline $\mathrm{Fe}(2)$ & $28(1)$ & $36(1)$ & $31(1)$ & $3(1)$ & $4(1)$ & $9(1)$ \\
\hline Cl (1) & $22(1)$ & $24(1)$ & $30(1)$ & $7(1)$ & $5(1)$ & $12(1)$ \\
\hline$F(1)$ & $82(1)$ & $101(2)$ & $53(1)$ & $37(1)$ & $26(1)$ & $66(1)$ \\
\hline$F(2)$ & $37(1)$ & $51(1)$ & $54(1)$ & $5(1)$ & $16(1)$ & $14(1)$ \\
\hline$F(3)$ & $61(1)$ & $31(1)$ & $69(1)$ & $-2(1)$ & $20(1)$ & $13(1)$ \\
\hline F (4) & $176(3)$ & $61(2)$ & $39(2)$ & $28(1)$ & $36(2)$ & $49(2)$ \\
\hline$F(5)$ & $79(2)$ & $101(2)$ & $107(2)$ & $77(2)$ & $63(2)$ & $71(2)$ \\
\hline$F(6)$ & $100(2)$ & $42(2)$ & $146(3)$ & $51(2)$ & $-66(2)$ & $-37(2)$ \\
\hline$F(7)$ & $34(1)$ & $173(3)$ & $70(2)$ & $21(2)$ & $1(1)$ & $-20(2)$ \\
\hline$F(8)$ & $63(2)$ & $67(2)$ & $206(3)$ & $26(2)$ & $-56(2)$ & $15(1)$ \\
\hline$F(9)$ & $49(1)$ & $93(2)$ & $66(2)$ & $-15(1)$ & $-9(1)$ & $-7(1)$ \\
\hline$F(10)$ & $181(4)$ & $56(2)$ & $103(3)$ & $5(2)$ & $17(3)$ & $-45(2)$ \\
\hline$F(11)$ & $57(2)$ & $128(2)$ & $214(4)$ & $122(3)$ & $16(2)$ & $-16(2)$ \\
\hline$F(12)$ & $125(3)$ & $97(2)$ & $76(2)$ & $67(2)$ & $-37(2)$ & $-57(2)$ \\
\hline$F(13)$ & $42(1)$ & $53(1)$ & $70(1)$ & $14(1)$ & $22(1)$ & $1(1)$ \\
\hline$F(14)$ & $30(1)$ & $112(2)$ & $75(2)$ & $29(1)$ & $5(1)$ & $27(1)$ \\
\hline$F(15)$ & $49(1)$ & $73(2)$ & $72(2)$ & $-21(1)$ & $31(1)$ & $1(1)$ \\
\hline$F(16)$ & $71(1)$ & $53(1)$ & $46(1)$ & $6(1)$ & $-18(1)$ & $-17(1)$ \\
\hline$F(17)$ & $59(1)$ & $23(1)$ & $111(2)$ & $4(1)$ & $-19(1)$ & $5(1)$ \\
\hline$F(18)$ & $59(1)$ & $51(1)$ & $75(2)$ & $-10(1)$ & $23(1)$ & $-23(1)$ \\
\hline$F(19)$ & $79(2)$ & $69(2)$ & $113(2)$ & $11(2)$ & $-41(2)$ & $23(1)$ \\
\hline$F(20)$ & $46(1)$ & $144(2)$ & $136(2)$ & $90(2)$ & $29(1)$ & $21(1)$ \\
\hline$F(21)$ & $71(2)$ & $132(2)$ & $45(1)$ & $22(1)$ & $-7(1)$ & $28(1)$ \\
\hline$F(22)$ & $99(2)$ & $40(1)$ & $93(2)$ & $-13(1)$ & $-19(2)$ & $-10(1)$ \\
\hline$F(23)$ & $194(3)$ & $51(2)$ & $55(2)$ & $-1(1)$ & $54(2)$ & $23(2)$ \\
\hline$F(24)$ & $88(2)$ & $37(1)$ & $59(1)$ & $-2(1)$ & $5(1)$ & $16(1)$ \\
\hline$O(1)$ & $37(1)$ & $61(2)$ & $54(2)$ & $18(1)$ & $0(1)$ & $-12(1)$ \\
\hline$O(2)$ & $84(2)$ & $58(2)$ & $58(2)$ & $8(1)$ & $21(1)$ & $40(1)$ \\
\hline$O(3)$ & $99(2)$ & $39(2)$ & $90(2)$ & $12(1)$ & $53(2)$ & $12(1)$ \\
\hline$O(4)$ & $49(2)$ & $99(2)$ & $42(2)$ & $10(2)$ & $-6(1)$ & $14(1)$ \\
\hline$C(1)$ & $30(2)$ & $45(2)$ & $33(2)$ & $3(2)$ & $-2(1)$ & $0(1)$ \\
\hline$C(2)$ & $47(2)$ & $42(2)$ & $41(2)$ & $8(2)$ & $5(2)$ & $16(2)$ \\
\hline$C(3)$ & $38(2)$ & $35(2)$ & $22(2)$ & $2(1)$ & $6(1)$ & $6(1)$ \\
\hline$C(4)$ & $55(2)$ & $32(2)$ & $30(2)$ & $7(1)$ & $4(2)$ & $6(2)$ \\
\hline$C(5)$ & $48(2)$ & $54(2)$ & $28(2)$ & $8(2)$ & $-4(2)$ & $17(2)$ \\
\hline$C(6)$ & $46(2)$ & $42(2)$ & $31(2)$ & $1(2)$ & $-1(2)$ & $-1(2)$ \\
\hline$C(7)$ & $46(2)$ & $30(2)$ & $28(2)$ & $1(1)$ & $5(1)$ & $10(1)$ \\
\hline$C(8)$ & $23(1)$ & $19(1)$ & $29(2)$ & $1(1)$ & $1(1)$ & $4(1)$ \\
\hline$C(9)$ & $26(1)$ & $24(2)$ & $26(2)$ & $4(1)$ & $6(1)$ & $8(1)$ \\
\hline C (10) & $33(2)$ & $25(2)$ & $26(2)$ & $4(1)$ & $6(1)$ & $9(1)$ \\
\hline C (11) & $28(2)$ & $22(2)$ & $34(2)$ & $2(1)$ & $1(1)$ & $7(1)$ \\
\hline$C(12)$ & $31(2)$ & $22(2)$ & $33(2)$ & $3(1)$ & $3(1)$ & $1(1)$ \\
\hline$C(13)$ & $27(1)$ & $22(2)$ & $34(2)$ & $6(1)$ & $7(1)$ & $7(1)$ \\
\hline$C(14)$ & $26(1)$ & $23(2)$ & $31(2)$ & $6(1)$ & $7(1)$ & $5(1)$ \\
\hline$C(15)$ & $30(2)$ & $33(2)$ & $40(2)$ & $8(1)$ & $8(1)$ & $6(1)$ \\
\hline$C(16)$ & $28(2)$ & $24(2)$ & $35(2)$ & $7(1)$ & $5(1)$ & $4(1)$ \\
\hline$C(17)$ & $40(2)$ & $36(2)$ & $32(2)$ & $8(1)$ & $7(1)$ & $2(1)$ \\
\hline$C(18)$ & $40(2)$ & $28(2)$ & $33(2)$ & $0(1)$ & $-2(1)$ & $3(1)$ \\
\hline
\end{tabular}




\begin{tabular}{|c|c|c|c|c|c|c|}
\hline C (19) & $47(2)$ & $50(2)$ & $54(2)$ & $-5(2)$ & $-13(2)$ & $0(2)$ \\
\hline C (20) & $70(2)$ & $45(2)$ & $29(2)$ & $3(2)$ & $-5(2)$ & $-3(2)$ \\
\hline$C(21)$ & $82(3)$ & $29(2)$ & $37(2)$ & $-3(2)$ & $-4(2)$ & $10(2)$ \\
\hline C (22) & $36(2)$ & $25(2)$ & $33(2)$ & $7(1)$ & $7(1)$ & $1(1)$ \\
\hline C (23) & $43(2)$ & $64(2)$ & $51(2)$ & $21(2)$ & $21(2)$ & $6(2)$ \\
\hline$C(24)$ & $60(2)$ & $27(2)$ & $33(2)$ & $8(1)$ & $8(2)$ & $-2(2)$ \\
\hline C (25) & $68(2)$ & $29(2)$ & $35(2)$ & $10(2)$ & $10(2)$ & $8(2)$ \\
\hline$C(26)$ & $45(2)$ & $42(2)$ & $50(2)$ & $8(2)$ & $21(2)$ & $7(2)$ \\
\hline$C(27)$ & $42(2)$ & $56(2)$ & $33(2)$ & $7(2)$ & $7(2)$ & $13(2)$ \\
\hline$C(28)$ & $26(2)$ & 71 (3) & $62(3)$ & $14(2)$ & $7(2)$ & $13(2)$ \\
\hline C (29) & $43(2)$ & $74(3)$ & $43(2)$ & $4(2)$ & $9(2)$ & $28(2)$ \\
\hline$C(30)$ & $45(2)$ & $45(2)$ & $60(3)$ & $-8(2)$ & $2(2)$ & $24(2)$ \\
\hline C (31) & $45(2)$ & $48(2)$ & $43(2)$ & $10(2)$ & $5(2)$ & $23(2)$ \\
\hline C (32) & $35(2)$ & $55(2)$ & $39(2)$ & $0(2)$ & $-3(2)$ & $14(2)$ \\
\hline$C(33)$ & $26(2)$ & $24(2)$ & $35(2)$ & $4(1)$ & $2(1)$ & $5(1)$ \\
\hline C (34) & $28(2)$ & $21(2)$ & $35(2)$ & $2(1)$ & $1(1)$ & $8(1)$ \\
\hline C (35) & $26(2)$ & $24(2)$ & $49(2)$ & $9(1)$ & $4(1)$ & $5(1)$ \\
\hline$C(36)$ & $28(2)$ & $26(2)$ & $38(2)$ & $9(1)$ & $6(1)$ & $9(1)$ \\
\hline C (37) & $30(2)$ & $27(2)$ & $34(2)$ & $5(1)$ & $3(1)$ & $9(1)$ \\
\hline C (38) & $24(1)$ & $22(2)$ & $32(2)$ & $5(1)$ & $3(1)$ & $6(1)$ \\
\hline C (39) & $37(2)$ & $24(2)$ & $38(2)$ & $2(1)$ & $-4(1)$ & $4(1)$ \\
\hline$C(40)$ & $74(3)$ & $28(2)$ & $50(2)$ & $-3(2)$ & $-2(2)$ & $-2(2)$ \\
\hline$C(41)$ & $51(2)$ & $36(2)$ & $36(2)$ & $-5(2)$ & $2(2)$ & $1(2)$ \\
\hline$C(42)$ & $43(2)$ & $51(2)$ & $50(2)$ & $3(2)$ & $-15(2)$ & $3(2)$ \\
\hline$C(43)$ & $29(2)$ & $29(2)$ & $49(2)$ & $14(1)$ & $11(1)$ & $7(1)$ \\
\hline C ( 44$)$ & $43(2)$ & $40(2)$ & $57(2)$ & $22(2)$ & $19(2)$ & $10(2)$ \\
\hline$C(45)$ & $35(2)$ & $46(2)$ & $66(2)$ & $19(2)$ & $17(2)$ & $7(2)$ \\
\hline$C(46)$ & $56(2)$ & $45(2)$ & $45(2)$ & $14(2)$ & $18(2)$ & $4(2)$ \\
\hline C ( 4 7) & $25(2)$ & $23(2)$ & $39(2)$ & $4(1)$ & $2(1)$ & $5(1)$ \\
\hline$C(48)$ & $32(2)$ & $35(2)$ & $44(2)$ & $1(2)$ & $0(1)$ & $4(1)$ \\
\hline C ( 49$)$ & $46(2)$ & $35(2)$ & $39(2)$ & $-1(2)$ & $3(2)$ & $-5(2)$ \\
\hline$C(50)$ & $34(2)$ & $25(2)$ & $38(2)$ & $4(1)$ & $1(1)$ & $0(1)$ \\
\hline C (51) & $33(2)$ & $21(2)$ & $33(2)$ & $0(1)$ & $5(1)$ & $4(1)$ \\
\hline C ( 52$)$ & $39(2)$ & $22(2)$ & $34(2)$ & $4(1)$ & $5(1)$ & $6(1)$ \\
\hline C (53) & $32(2)$ & $25(2)$ & 31 (2) & $1(1)$ & $6(1)$ & $9(1)$ \\
\hline$C(54)$ & $29(2)$ & $30(2)$ & $32(2)$ & $3(1)$ & $4(1)$ & $3(1)$ \\
\hline C (55) & $31(2)$ & $23(2)$ & $34(2)$ & $5(1)$ & $6(1)$ & $5(1)$ \\
\hline$C(56)$ & $31(2)$ & $24(2)$ & $35(2)$ & $1(1)$ & $6(1)$ & $7(1)$ \\
\hline$C(57)$ & $43(2)$ & $45(2)$ & $37(2)$ & $9(2)$ & $12(2)$ & $16(2)$ \\
\hline C (58) & $21(2)$ & $43(2)$ & $55(2)$ & $12(2)$ & $8(1)$ & $6(1)$ \\
\hline$C(59)$ & $30(2)$ & $25(2)$ & $33(2)$ & $0(1)$ & $10(1)$ & $3(1)$ \\
\hline$C(60)$ & $32(2)$ & $28(2)$ & $36(2)$ & $-1(1)$ & $9(1)$ & $3(1)$ \\
\hline C (61) & $32(2)$ & $31(2)$ & $32(2)$ & $0(1)$ & $4(1)$ & $7(1)$ \\
\hline$C(62)$ & $47(2)$ & $34(2)$ & $31(2)$ & $4(1)$ & $1(1)$ & $13(1)$ \\
\hline$C(63)$ & $42(2)$ & $28(2)$ & $35(2)$ & $6(1)$ & $4(1)$ & $0(1)$ \\
\hline$C(64)$ & $30(2)$ & $32(2)$ & $29(2)$ & $1(1)$ & $3(1)$ & $3(1)$ \\
\hline$C(65)$ & $31(2)$ & $48(2)$ & $58(2)$ & $4(2)$ & $3(2)$ & $4(2)$ \\
\hline$C(66)$ & $66(3)$ & 41 (2) & $53(2)$ & $15(2)$ & $-3(2)$ & $-12(2)$ \\
\hline$C(67)$ & $28(2)$ & $25(2)$ & $28(2)$ & $3(1)$ & $2(1)$ & $2(1)$ \\
\hline$C(68)$ & $29(2)$ & $25(2)$ & $33(2)$ & $2(1)$ & $1(1)$ & $2(1)$ \\
\hline C (69) & $24(1)$ & $29(2)$ & $31(2)$ & $6(1)$ & $2(1)$ & $7(1)$ \\
\hline$C(70)$ & $36(2)$ & $25(2)$ & $27(2)$ & $2(1)$ & $4(1)$ & $13(1)$ \\
\hline$C(71)$ & $33(2)$ & $27(2)$ & $29(2)$ & $4(1)$ & $1(1)$ & $5(1)$ \\
\hline$C(72)$ & $28(2)$ & $27(2)$ & $34(2)$ & $8(1)$ & $7(1)$ & $5(1)$ \\
\hline C (73) & $34(2)$ & $46(2)$ & $37(2)$ & $-1(2)$ & $6(1)$ & $9(2)$ \\
\hline$C(74)$ & $36(2)$ & $29(2)$ & $42(2)$ & $2(2)$ & $3(2)$ & $4(1)$ \\
\hline$C(75)$ & $36(2)$ & $26(2)$ & $31(2)$ & $3(1)$ & $9(1)$ & $3(1)$ \\
\hline$C(76)$ & $36(2)$ & $32(2)$ & 31 (2) & $9(1)$ & $6(1)$ & $2(1)$ \\
\hline$C(77)$ & $39(2)$ & $39(2)$ & $32(2)$ & $7(2)$ & $2(1)$ & $-2(1)$ \\
\hline C (78) & $48(2)$ & $41(2)$ & $35(2)$ & $7(2)$ & $-1(2)$ & $-9(2)$ \\
\hline C (79) & $57(2)$ & $33(2)$ & $30(2)$ & $3(2)$ & $5(2)$ & $-3(2)$ \\
\hline
\end{tabular}




\begin{tabular}{|c|c|c|c|c|c|c|}
\hline$C(80)$ & $41(2)$ & $32(2)$ & $32(2)$ & $6(1)$ & $4(1)$ & $4(1)$ \\
\hline$C(81)$ & $46(2)$ & $50(2)$ & $44(2)$ & $13(2)$ & $-1(2)$ & $-2(2)$ \\
\hline$C(82)$ & $87(3)$ & $38(2)$ & $37(2)$ & $-2(2)$ & $10(2)$ & $-4(2)$ \\
\hline B (1) & $27(2)$ & $24(2)$ & $35(2)$ & $2(2)$ & $6(1)$ & $3(1)$ \\
\hline
\end{tabular}


Table 3.5 Hydrogen coordinates ( $\left.x 1^{\wedge} 4\right)$ and isotropic displacement parameters $\left(A^{\wedge} 2 \times 10^{\wedge} 3\right)$ for 10 .

\begin{tabular}{|c|c|c|c|c|}
\hline & $\mathrm{x}$ & $\mathrm{Y}$ & z & $\mathrm{U}(\mathrm{eq})$ \\
\hline $\mathrm{H}(3)$ & 1125 & 1284 & 805 & 38 \\
\hline $\mathrm{H}(4)$ & -48 & 2464 & 604 & 47 \\
\hline $\mathrm{H}(5)$ & -1790 & 1616 & 383 & 52 \\
\hline $\mathrm{H}(6)$ & -1698 & -68 & 493 & 50 \\
\hline $\mathrm{H}(7)$ & 109 & -277 & 785 & 42 \\
\hline $\mathrm{H}(10)$ & 1663 & 2376 & 4566 & 33 \\
\hline $\mathrm{H}(12)$ & 3077 & 4225 & 3679 & 35 \\
\hline $\mathrm{H}(15 \mathrm{~A})$ & -987 & 991 & 3435 & 50 \\
\hline $\mathrm{H}(15 \mathrm{~B})$ & -545 & 1724 & 3029 & 50 \\
\hline $\mathrm{H}(15 \mathrm{C})$ & -577 & 2045 & 3780 & 50 \\
\hline $\mathrm{H}(16 \mathrm{~A})$ & 1363 & 174 & 3235 & 43 \\
\hline $\mathrm{H}(16 \mathrm{~B})$ & 627 & 487 & 2690 & 43 \\
\hline $\mathrm{H}(16 \mathrm{C})$ & 206 & -175 & 3149 & 43 \\
\hline $\mathrm{H}(17 \mathrm{~A})$ & 1277 & 737 & 4374 & 54 \\
\hline $\mathrm{H}(17 \mathrm{~B})$ & 125 & 355 & 4228 & 54 \\
\hline $\mathrm{H}(17 \mathrm{C})$ & 478 & 1418 & 4579 & 54 \\
\hline $\mathrm{H}(19 \mathrm{~A})$ & 4188 & 3232 & 4903 & 82 \\
\hline $\mathrm{H}(19 \mathrm{~B})$ & 4414 & 4270 & 5321 & 82 \\
\hline $\mathrm{H}(19 \mathrm{C})$ & 4332 & 4123 & 4556 & 82 \\
\hline $\mathrm{H}(20 \mathrm{~A})$ & 1871 & 3654 & 5414 & 75 \\
\hline $\mathrm{H}(20 \mathrm{~B})$ & 2922 & 4015 & 5841 & 75 \\
\hline $\mathrm{H}(20 \mathrm{C})$ & 2711 & 2966 & 5440 & 75 \\
\hline $\mathrm{H}(21 \mathrm{~A})$ & 3070 & 5253 & 4555 & 77 \\
\hline $\mathrm{H}(21 \mathrm{~B})$ & 3124 & 5370 & 5317 & 77 \\
\hline $\mathrm{H}(21 \mathrm{C})$ & 2086 & 5056 & 4865 & 77 \\
\hline $\mathrm{H}(23 \mathrm{~A})$ & 3789 & 3068 & 2467 & 76 \\
\hline $\mathrm{H}(23 \mathrm{~B})$ & 3919 & 4110 & 2877 & 76 \\
\hline $\mathrm{H}(23 \mathrm{C})$ & 3755 & 3953 & 2110 & 76 \\
\hline $\mathrm{H}(24 \mathrm{~A})$ & 2158 & 2476 & 1758 & 60 \\
\hline $\mathrm{H}(24 \mathrm{~B})$ & 2143 & 3447 & 1496 & 60 \\
\hline $\mathrm{H}(24 \mathrm{C})$ & 1234 & 3058 & 1820 & 60 \\
\hline $\mathrm{H}(25 \mathrm{~A})$ & 2320 & 4923 & 2186 & 65 \\
\hline $\mathrm{H}(25 \mathrm{~B})$ & 2484 & 5020 & 2951 & 65 \\
\hline $\mathrm{H}(25 \mathrm{C})$ & 1427 & 4605 & 2549 & 65 \\
\hline $\mathrm{H}(28)$ & 6692 & 592 & 1530 & 62 \\
\hline $\mathrm{H}(29)$ & 5877 & -715 & 651 & 63 \\
\hline $\mathrm{H}(30)$ & 4933 & -1915 & 1158 & 62 \\
\hline $\mathrm{H}(31)$ & 5212 & -1361 & 2359 & 53 \\
\hline $\mathrm{H}(32)$ & 6279 & 220 & 2594 & 53 \\
\hline $\mathrm{H}(35)$ & 1292 & -2445 & 3094 & 39 \\
\hline $\mathrm{H}(37)$ & 2953 & -466 & 4436 & 36 \\
\hline $\mathrm{H}(40 \mathrm{~A})$ & 1591 & -3515 & 1558 & 79 \\
\hline $\mathrm{H}(40 \mathrm{~B})$ & 1288 & -3392 & 2264 & 79 \\
\hline $\mathrm{H}(40 \mathrm{C})$ & 2428 & -3338 & 2170 & 79 \\
\hline $\mathrm{H}(41 \mathrm{~A})$ & 2420 & -1229 & 1472 & 65 \\
\hline $\mathrm{H}(41 \mathrm{~B})$ & 2194 & -2298 & 1108 & 65 \\
\hline $\mathrm{H}(41 \mathrm{C})$ & 3133 & -1984 & 1652 & 65 \\
\hline $\mathrm{H}(42 \mathrm{~A})$ & 787 & -1201 & 1839 & 76 \\
\hline $\mathrm{H}(42 \mathrm{~B})$ & 339 & -2063 & 2139 & 76 \\
\hline $\mathrm{H}(42 \mathrm{C})$ & 505 & -2221 & 1402 & 76 \\
\hline $\mathrm{H}(44 \mathrm{~A})$ & 1833 & -3324 & 3978 & 66 \\
\hline $\mathrm{H}(4 \mathrm{BB})$ & 1683 & -3215 & 4717 & 66 \\
\hline $\mathrm{H}(44 \mathrm{C})$ & 2705 & -2801 & 4525 & 66 \\
\hline
\end{tabular}




\begin{tabular}{|c|c|c|c|c|}
\hline $\mathrm{H}(45 \mathrm{~A})$ & 193 & -1540 & 4170 & 70 \\
\hline $\mathrm{H}(45 \mathrm{~B})$ & 165 & -2435 & 4519 & 70 \\
\hline $\mathrm{H}(45 \mathrm{C})$ & 272 & -2559 & 3773 & 70 \\
\hline $\mathrm{H}(46 \mathrm{~A})$ & 2549 & -1231 & 5148 & 71 \\
\hline $\mathrm{H}(46 \mathrm{~B})$ & 1534 & -1658 & 5343 & 71 \\
\hline $\mathrm{H}(46 \mathrm{C})$ & 1566 & -744 & 5013 & 71 \\
\hline $\mathrm{H}(48 \mathrm{~A})$ & 5050 & -111 & 3273 & 57 \\
\hline $\mathrm{H}(48 \mathrm{~B})$ & 5135 & -266 & 4002 & 57 \\
\hline $\mathrm{H}(48 \mathrm{C})$ & 5523 & 733 & 3842 & 57 \\
\hline $\mathrm{H}(49 \mathrm{~A})$ & 4488 & 1514 & 4580 & 62 \\
\hline $\mathrm{H}(49 \mathrm{~B})$ & 4126 & 511 & 4744 & 62 \\
\hline $\mathrm{H}(49 \mathrm{C})$ & 3333 & 1166 & 4512 & 62 \\
\hline $\mathrm{H}(50 \mathrm{~A})$ & 4006 & 1067 & 2930 & 49 \\
\hline $\mathrm{H}(50 \mathrm{~B})$ & 4240 & 1853 & 3566 & 49 \\
\hline $\mathrm{H}(50 \mathrm{C})$ & 3131 & 1374 & 3318 & 49 \\
\hline $\mathrm{H}(52)$ & 8562 & -5289 & 1764 & 38 \\
\hline $\mathrm{H}(54)$ & 9337 & -3746 & 452 & 37 \\
\hline $\mathrm{H}(56)$ & 7180 & -3026 & 1505 & 37 \\
\hline $\mathrm{H}(60)$ & 8765 & -4395 & 2943 & 39 \\
\hline $\mathrm{H}(62)$ & 8423 & -6522 & 3933 & 45 \\
\hline $\mathrm{H}(64)$ & 6168 & -6065 & 2657 & 37 \\
\hline $\mathrm{H}(68)$ & 5263 & -4214 & 2752 & 35 \\
\hline $\mathrm{H}(70)$ & 5972 & -1652 & 3882 & 34 \\
\hline $\mathrm{H}(72)$ & 7869 & -2694 & 2721 & 35 \\
\hline $\mathrm{H}(76)$ & 5093 & -4126 & 1592 & 39 \\
\hline $\mathrm{H}(78)$ & 4182 & -6500 & 290 & 51 \\
\hline $\mathrm{H}(80)$ & 6718 & -6297 & 1512 & 42 \\
\hline
\end{tabular}




\section{Details of crystal structure of compound 14}

Table 4.1 Crystal data and structure refinement for $\mathbf{1 4}$.

Empirical formula

Formula weight

Temperature

Wavelength

Crystal system

Space group

Unit cell dimensions

\section{Volume}

Z

Density (calculated)

Absorption coefficient

$\mathrm{F}(000)$

Crystal size

Theta range for data collection

Index ranges

Reflections collected

Independent reflections

Completeness to theta $=26.37^{\circ}$

Absorption correction

Max. and min. transmission

Refinement method

Data / restraints / parameters

Goodness-of-fit on $\mathrm{F}^{2}$

Final R indices [I $>2 \operatorname{sigma}(\mathrm{I})]$

$\mathrm{R}$ indices (all data)

Absolute structure parameter

Largest diff. peak and hole
C60 H50 B F24 Fe2 In O5

1544.33

$150(2) \mathrm{K}$

$0.71073 \AA$

Orthorhombic

P 212121

$\mathrm{a}=16.0540(3) \AA \quad \alpha=90^{\circ}$.

$\mathrm{b}=16.2940(3) \AA \quad \beta=90^{\circ}$.

$\mathrm{c}=24.2520(6) \AA \quad \gamma=90^{\circ}$.

6343.9(2) $\AA^{3}$

4

$1.617 \mathrm{Mg} / \mathrm{m}^{3}$

$0.929 \mathrm{~mm}^{-1}$

3088

$0.23 \times 0.15 \times 0.10 \mathrm{~mm}^{3}$

3.55 to $26.37^{\circ}$.

$-20<=\mathrm{h}<=20,-20<=\mathrm{k}<=20,-29<=1<=30$

26498

$12739[\mathrm{R}($ int $)=0.0692]$

$99.5 \%$

Semi-empirical from equivalents

0.9128 and 0.8147

Full-matrix least-squares on $\mathrm{F}^{2}$

12739 / 150 / 857

1.023

$\mathrm{R} 1=0.0821, \mathrm{wR} 2=0.1815$

$\mathrm{R} 1=0.1226, \mathrm{wR} 2=0.2068$

$-0.03(3)$

1.154 and -1.007 e. $\AA^{-3}$ 
Table 4.2 Atomic coordinates ( x 10 $)$ and equivalent isotropic displacement parameters $\left(\AA^{2} \times 10^{3}\right)$ for 14 .

$\mathrm{U}(\mathrm{eq})$ is defined as one third of the trace of the orthogonalized $\mathrm{U}^{\mathrm{ij}}$ tensor.

\begin{tabular}{|c|c|c|c|c|}
\hline & $\mathrm{X}$ & $\mathrm{y}$ & $\mathrm{z}$ & $\mathrm{U}(\mathrm{eq})$ \\
\hline $\mathrm{C}(1)$ & $6310(6)$ & $4233(5)$ & $6421(4)$ & $36(2)$ \\
\hline $\mathrm{C}(2)$ & $5711(7)$ & $4200(6)$ & $5995(5)$ & $51(3)$ \\
\hline $\mathrm{C}(3)$ & $4940(7)$ & $4509(6)$ & $6223(6)$ & $56(3)$ \\
\hline $\mathrm{C}(4)$ & $5064(7)$ & $4708(6)$ & $6769(5)$ & $50(3)$ \\
\hline $\mathrm{C}(5)$ & $5905(7)$ & $4551(6)$ & $6903(5)$ & $44(3)$ \\
\hline$C(6)$ & $7141(6)$ & $3850(6)$ & $6395(5)$ & $52(3)$ \\
\hline $\mathrm{C}(7)$ & $5812(11)$ & $3879(8)$ & $5430(5)$ & $80(4)$ \\
\hline $\mathrm{C}(8)$ & $4123(10)$ & $4525(10)$ & $5911(7)$ & $95(5)$ \\
\hline $\mathrm{C}(9)$ & $4395(10)$ & 4981(9) & $7178(6)$ & $79(4)$ \\
\hline $\mathrm{C}(10)$ & $6304(9)$ & $4634(8)$ & $7466(5)$ & $68(4)$ \\
\hline $\mathrm{C}(11)$ & $9583(6)$ & $7292(7)$ & $6646(5)$ & $49(3)$ \\
\hline$C(12)$ & $8869(7)$ & $7590(6)$ & $6366(4)$ & $41(2)$ \\
\hline $\mathrm{C}(13)$ & $8154(7)$ & $7467(6)$ & $6692(4)$ & $41(2)$ \\
\hline $\mathrm{C}(14)$ & $8402(8)$ & $7085(7)$ & $7189(4)$ & $49(3)$ \\
\hline$C(15)$ & $9314(8)$ & $6986(6)$ & $7155(5)$ & $52(3)$ \\
\hline$C(16)$ & $10456(10)$ & $7349(10)$ & $6469(7)$ & $87(5)$ \\
\hline $\mathrm{C}(17)$ & 8864(11) & $8021(8)$ & $5804(5)$ & $81(5)$ \\
\hline $\mathrm{C}(18)$ & $7324(8)$ & $7830(6)$ & $6566(6)$ & $65(4)$ \\
\hline $\mathrm{C}(19)$ & 7881(9) & $6863(9)$ & $7668(6)$ & $76(4)$ \\
\hline $\mathrm{C}(20)$ & $9816(9)$ & $6669(9)$ & $7610(6)$ & $77(4)$ \\
\hline $\mathrm{C}(21)$ & $9200(7)$ & $6029(8)$ & $5920(5)$ & $62(3)$ \\
\hline $\mathrm{C}(22)$ & $8841(7)$ & $5378(7)$ & $6852(5)$ & $51(3)$ \\
\hline $\mathrm{C}(23)$ & $5597(7)$ & $5956(8)$ & $5675(5)$ & $57(3)$ \\
\hline $\mathrm{C}(24)$ & $5695(7)$ & $6274(7)$ & $6704(5)$ & $51(3)$ \\
\hline $\mathrm{C}(25)$ & $7882(6)$ & $1616(5)$ & $5695(4)$ & $33(2)$ \\
\hline$C(26)$ & $7486(6)$ & $1762(5)$ & $5190(4)$ & $33(2)$ \\
\hline $\mathrm{C}(27)$ & $7771(6)$ & $2398(6)$ & $4838(3)$ & $36(2)$ \\
\hline$C(28)$ & $8430(6)$ & $2877(6)$ & 4982(4) & $35(2)$ \\
\hline C(29) & $8818(6)$ & $2739(6)$ & $5483(4)$ & $35(2)$ \\
\hline$C(30)$ & $8543(6)$ & $2136(6)$ & $5837(4)$ & $39(2)$ \\
\hline $\mathrm{C}(31)$ & $7355(7)$ & $2518(6)$ & 4301(4) & $44(2)$ \\
\hline$C(32)$ & $9521(8)$ & $3280(7)$ & $5667(6)$ & $55(3)$ \\
\hline$C(33)$ & $6937(5)$ & $1432(5)$ & $6548(3)$ & $25(2)$ \\
\hline$C(34)$ & 7191(6) & $1728(5)$ & $7063(3)$ & $30(2)$ \\
\hline $\mathrm{C}(35)$ & $6702(6)$ & 2192(6) & $7407(4)$ & $34(2)$ \\
\hline$C(36)$ & $5890(6)$ & $2379(5)$ & $7259(4)$ & $31(2)$ \\
\hline$C(37)$ & $5589(6)$ & $2107(5)$ & $6763(4)$ & $30(2)$ \\
\hline C(39) & $7050(7)$ & $2436(7)$ & $7946(4)$ & $44(3)$ \\
\hline $\mathrm{C}(38)$ & $6111(6)$ & $1639(5)$ & $6407(4)$ & $36(2)$ \\
\hline$C(40)$ & $4718(7)$ & $2238(6)$ & $6586(4)$ & $41(2)$ \\
\hline$C(41)$ & $8293(6)$ & $447(5)$ & $6456(3)$ & $27(2)$ \\
\hline$C(42)$ & $8158(6)$ & $42(5)$ & $6962(4)$ & $31(2)$ \\
\hline$C(43)$ & $8770(7)$ & $-384(6)$ & $7236(4)$ & $36(2)$ \\
\hline$C(44)$ & $9548(6)$ & $-471(6)$ & 7018(4) & $35(2)$ \\
\hline$C(45)$ & $9702(6)$ & $-88(6)$ & $6513(4)$ & $37(2)$ \\
\hline$C(46)$ & $9089(6)$ & $353(5)$ & $6246(4)$ & $32(2)$ \\
\hline $\mathrm{C}(47)$ & $8561(8)$ & $-741(7)$ & 7799(4) & $51(3)$ \\
\hline$C(48)$ & $10541(7)$ & $-136(6)$ & $6266(5)$ & $49(3)$ \\
\hline C(49) & $7022(6)$ & $169(6)$ & $5818(4)$ & $32(2)$ \\
\hline$C(50)$ & $6333(6)$ & $-222(6)$ & $6057(4)$ & $37(2)$ \\
\hline $\mathrm{C}(51)$ & $5938(6)$ & $-877(6)$ & $5823(4)$ & $36(2)$ \\
\hline$C(52)$ & $6236(6)$ & $-1198(6)$ & $5318(4)$ & $37(2)$ \\
\hline$C(53)$ & $6924(6)$ & $-833(6)$ & $5085(3)$ & $36(2)$ \\
\hline$C(54)$ & $7307(6)$ & $-176(5)$ & $5330(3)$ & $33(2)$ \\
\hline
\end{tabular}




\begin{tabular}{|c|c|c|c|c|}
\hline$C(55)$ & $5219(6)$ & $-1224(6)$ & $6096(5)$ & $47(3)$ \\
\hline$C(56)$ & $7272(10)$ & $-1170(7)$ & 4543(4) & $58(3)$ \\
\hline$C(57)$ & $7661(10)$ & $5068(8)$ & $4882(5)$ & $71(4)$ \\
\hline $\mathrm{C}(58)$ & $7824(12)$ & $5277(10)$ & $4359(7)$ & $99(5)$ \\
\hline C(59) & $7664(10)$ & $6153(9)$ & $4337(5)$ & $76(4)$ \\
\hline$C(60)$ & $7532(13)$ & $6524(11)$ & $4906(7)$ & $104(5)$ \\
\hline $\mathrm{B}(1)$ & $7526(6)$ & $929(6)$ & $6135(4)$ & $32(2)$ \\
\hline $\mathrm{F}(1 \mathrm{~A})$ & $7646(10)$ & $2053(9)$ & $3925(6)$ & $72(4)$ \\
\hline$F(1 B)$ & $7118(9)$ & 1833(8) & $4037(6)$ & $55(3)$ \\
\hline $\mathrm{F}(2)$ & $6584(6)$ & $2780(6)$ & $4355(4)$ & $100(3)$ \\
\hline $\mathrm{F}(3)$ & $7648(6)$ & $3166(6)$ & $4023(4)$ & $106(3)$ \\
\hline $\mathrm{F}(4)$ & $9240(6)$ & $4040(6)$ & $5814(4)$ & $102(3)$ \\
\hline $\mathrm{F}(5)$ & $10035(6)$ & $3453(5)$ & $5265(4)$ & $88(2)$ \\
\hline $\mathrm{F}(6)$ & 9963(5) & $3019(5)$ & $6077(3)$ & $83(2)$ \\
\hline$F(7)$ & $7410(6)$ & $1847(5)$ & $8221(3)$ & $86(3)$ \\
\hline $\mathrm{F}(8)$ & $6472(5)$ & $2760(5)$ & $8286(3)$ & $74(2)$ \\
\hline $\mathrm{F}(9)$ & $7627(5)$ & $3032(5)$ & $7902(3)$ & $75(2)$ \\
\hline $\mathrm{F}(10)$ & $4250(4)$ & $1568(4)$ & $6655(3)$ & $59(2)$ \\
\hline $\mathrm{F}(11)$ & $4341(4)$ & $2828(4)$ & $6882(3)$ & $56(2)$ \\
\hline$F(12)$ & $4645(4)$ & $2447(4)$ & $6063(3)$ & $59(2)$ \\
\hline$F(13)$ & $8705(6)$ & $-173(5)$ & 8181(4) & $87(2)$ \\
\hline $\mathrm{F}(14)$ & $7791(5)$ & $-1003(6)$ & $7839(3)$ & $89(3)$ \\
\hline $\mathrm{F}(15)$ & $9066(5)$ & $-1368(4)$ & $7925(3)$ & $67(2)$ \\
\hline$F(16)$ & $11114(6)$ & $-467(6)$ & $6585(4)$ & $94(3)$ \\
\hline$F(17)$ & $10554(5)$ & $-570(5)$ & $5799(3)$ & $88(2)$ \\
\hline $\mathrm{F}(18)$ & $10846(6)$ & 591(5) & $6119(4)$ & $96(3)$ \\
\hline$F(19)$ & $6910(5)$ & $-1869(5)$ & $4390(3)$ & $83(2)$ \\
\hline $\mathrm{F}(20)$ & $8069(5)$ & $-1308(5)$ & $4568(3)$ & $68(2)$ \\
\hline $\mathrm{F}(21)$ & $7156(5)$ & $-625(5)$ & $4142(3)$ & $79(2)$ \\
\hline$F(22)$ & $4890(6)$ & $-1881(6)$ & $5866(4)$ & $100(3)$ \\
\hline $\mathrm{F}(23)$ & $5378(5)$ & $-1460(6)$ & $6630(3)$ & $87(3)$ \\
\hline$F(24)$ & $4594(4)$ & $-726(5)$ & $6180(4)$ & $95(3)$ \\
\hline $\mathrm{Fe}(1)$ & $5847(1)$ & $5434(1)$ & $6282(1)$ & $38(1)$ \\
\hline $\mathrm{Fe}(2)$ & $8731(1)$ & 6331(1) & $6516(1)$ & $39(1)$ \\
\hline $\operatorname{In}(1)$ & $7338(1)$ & $5852(1)$ & $6195(1)$ & $33(1)$ \\
\hline $\mathrm{O}(1)$ & $9552(6)$ & $5871(7)$ & $5494(4)$ & $84(3)$ \\
\hline $\mathrm{O}(2)$ & $8917(6)$ & $4780(5)$ & $7085(4)$ & $74(3)$ \\
\hline $\mathrm{O}(3)$ & $5398(7)$ & $6298(6)$ & $5282(4)$ & $85(3)$ \\
\hline $\mathrm{O}(4)$ & $5590(6)$ & $6795(5)$ & $7028(4)$ & $71(3)$ \\
\hline $\mathrm{O}(5)$ & $7521(7)$ & $5712(6)$ & $5225(3)$ & $84(3)$ \\
\hline
\end{tabular}


Table 4.3 Bond lengths $\left[\AA \AA^{\circ}\right]$ and angles $\left[{ }^{\circ}\right]$ for $\mathbf{1 4}$.

\begin{tabular}{|c|c|}
\hline $\mathrm{C}(1)-\mathrm{C}(2)$ & $1.412(14)$ \\
\hline$C(1)-C(5)$ & $1.434(14)$ \\
\hline$C(1)-C(6)$ & $1.475(14)$ \\
\hline $\mathrm{C}(1)-\mathrm{Fe}(1)$ & $2.119(9)$ \\
\hline$C(2)-C(3)$ & $1.446(16)$ \\
\hline$C(2)-C(7)$ & $1.477(16)$ \\
\hline$C(2)-F e(1)$ & $2.138(10)$ \\
\hline$C(3)-C(4)$ & $1.378(17)$ \\
\hline $\mathrm{C}(3)-\mathrm{C}(8)$ & $1.515(19)$ \\
\hline $\mathrm{C}(3)-\mathrm{Fe}(1)$ & 2.101(10) \\
\hline$C(4)-C(5)$ & $1.411(16)$ \\
\hline$C(4)-C(9)$ & $1.528(18)$ \\
\hline $\mathrm{C}(4)-\mathrm{Fe}(1)$ & $2.092(11)$ \\
\hline$C(5)-C(10)$ & $1.513(16)$ \\
\hline $\mathrm{C}(5)-\mathrm{Fe}(1)$ & $2.085(10)$ \\
\hline $\mathrm{C}(6)-\mathrm{H}(6 \mathrm{~A})$ & 0.9800 \\
\hline $\mathrm{C}(6)-\mathrm{H}(6 \mathrm{~B})$ & 0.9800 \\
\hline $\mathrm{C}(6)-\mathrm{H}(6 \mathrm{C})$ & 0.9800 \\
\hline $\mathrm{C}(7)-\mathrm{H}(7 \mathrm{~A})$ & 0.9800 \\
\hline $\mathrm{C}(7)-\mathrm{H}(7 \mathrm{~B})$ & 0.9800 \\
\hline $\mathrm{C}(7)-\mathrm{H}(7 \mathrm{C})$ & 0.9800 \\
\hline $\mathrm{C}(8)-\mathrm{H}(8 \mathrm{~A})$ & 0.9800 \\
\hline $\mathrm{C}(8)-\mathrm{H}(8 \mathrm{~B})$ & 0.9800 \\
\hline $\mathrm{C}(8)-\mathrm{H}(8 \mathrm{C})$ & 0.9800 \\
\hline $\mathrm{C}(9)-\mathrm{H}(9 \mathrm{~A})$ & 0.9800 \\
\hline $\mathrm{C}(9)-\mathrm{H}(9 \mathrm{~B})$ & 0.9800 \\
\hline $\mathrm{C}(9)-\mathrm{H}(9 \mathrm{C})$ & 0.9800 \\
\hline $\mathrm{C}(10)-\mathrm{H}(10 \mathrm{~A})$ & 0.9800 \\
\hline $\mathrm{C}(10)-\mathrm{H}(10 \mathrm{~B})$ & 0.9800 \\
\hline $\mathrm{C}(10)-\mathrm{H}(10 \mathrm{C})$ & 0.9800 \\
\hline $\mathrm{C}(11)-\mathrm{C}(15)$ & $1.399(17)$ \\
\hline $\mathrm{C}(11)-\mathrm{C}(12)$ & $1.419(15)$ \\
\hline $\mathrm{C}(11)-\mathrm{C}(16)$ & $1.470(19)$ \\
\hline $\mathrm{C}(11)-\mathrm{Fe}(2)$ & $2.102(10)$ \\
\hline $\mathrm{C}(12)-\mathrm{C}(13)$ & $1.408(15)$ \\
\hline $\mathrm{C}(12)-\mathrm{C}(17)$ & $1.533(15)$ \\
\hline $\mathrm{C}(12)-\mathrm{Fe}(2)$ & $2.095(10)$ \\
\hline $\mathrm{C}(13)-\mathrm{C}(14)$ & $1.413(15)$ \\
\hline $\mathrm{C}(13)-\mathrm{C}(18)$ & $1.490(15)$ \\
\hline $\mathrm{C}(13)-\mathrm{Fe}(2)$ & $2.113(10)$ \\
\hline $\mathrm{C}(14)-\mathrm{C}(15)$ & $1.475(17)$ \\
\hline $\mathrm{C}(14)-\mathrm{C}(19)$ & $1.476(17)$ \\
\hline $\mathrm{C}(14)-\mathrm{Fe}(2)$ & $2.109(11)$ \\
\hline$C(15)-C(20)$ & $1.461(18)$ \\
\hline $\mathrm{C}(15)-\mathrm{Fe}(2)$ & $2.100(10)$ \\
\hline $\mathrm{C}(16)-\mathrm{H}(16 \mathrm{~A})$ & 0.9800 \\
\hline $\mathrm{C}(16)-\mathrm{H}(16 \mathrm{~B})$ & 0.9800 \\
\hline $\mathrm{C}(16)-\mathrm{H}(16 \mathrm{C})$ & 0.9800 \\
\hline $\mathrm{C}(17)-\mathrm{H}(17 \mathrm{~A})$ & 0.9800 \\
\hline $\mathrm{C}(17)-\mathrm{H}(17 \mathrm{~B})$ & 0.9800 \\
\hline $\mathrm{C}(17)-\mathrm{H}(17 \mathrm{C})$ & 0.9800 \\
\hline $\mathrm{C}(18)-\mathrm{H}(18 \mathrm{~A})$ & 0.9800 \\
\hline $\mathrm{C}(18)-\mathrm{H}(18 \mathrm{~B})$ & 0.9800 \\
\hline $\mathrm{C}(18)-\mathrm{H}(18 \mathrm{C})$ & 0.9800 \\
\hline $\mathrm{C}(19)-\mathrm{H}(19 \mathrm{~A})$ & 0.9800 \\
\hline $\mathrm{C}(19)-\mathrm{H}(19 \mathrm{~B})$ & 0.9800 \\
\hline $\mathrm{C}(19)-\mathrm{H}(19 \mathrm{C})$ & 0.9800 \\
\hline
\end{tabular}




\begin{tabular}{|c|c|}
\hline $\mathrm{C}(20)-\mathrm{H}(20 \mathrm{~A})$ & 0.9800 \\
\hline $\mathrm{C}(20)-\mathrm{H}(20 \mathrm{~B})$ & 0.9800 \\
\hline $\mathrm{C}(20)-\mathrm{H}(20 \mathrm{C})$ & 0.9800 \\
\hline $\mathrm{C}(21)-\mathrm{O}(1)$ & $1.205(13)$ \\
\hline $\mathrm{C}(21)-\mathrm{Fe}(2)$ & $1.703(11)$ \\
\hline $\mathrm{C}(22)-\mathrm{O}(2)$ & $1.132(13)$ \\
\hline $\mathrm{C}(22)-\mathrm{Fe}(2)$ & $1.763(11)$ \\
\hline $\mathrm{C}(23)-\mathrm{O}(3)$ & $1.149(14)$ \\
\hline $\mathrm{C}(23)-\mathrm{Fe}(1)$ & $1.748(13)$ \\
\hline $\mathrm{C}(24)-\mathrm{O}(4)$ & $1.169(13)$ \\
\hline $\mathrm{C}(24)-\mathrm{Fe}(1)$ & $1.727(13)$ \\
\hline$C(25)-C(26)$ & $1.400(13)$ \\
\hline$C(25)-C(30)$ & $1.400(14)$ \\
\hline $\mathrm{C}(25)-\mathrm{B}(1)$ & $1.648(13)$ \\
\hline $\mathrm{C}(26)-\mathrm{C}(27)$ & $1.418(13)$ \\
\hline $\mathrm{C}(26)-\mathrm{H}(26)$ & 0.9500 \\
\hline $\mathrm{C}(27)-\mathrm{C}(28)$ & $1.360(14)$ \\
\hline $\mathrm{C}(27)-\mathrm{C}(31)$ & $1.477(14)$ \\
\hline $\mathrm{C}(28)-\mathrm{C}(29)$ & $1.383(13)$ \\
\hline $\mathrm{C}(28)-\mathrm{H}(28)$ & 0.9500 \\
\hline $\mathrm{C}(29)-\mathrm{C}(30)$ & $1.378(13)$ \\
\hline $\mathrm{C}(29)-\mathrm{C}(32)$ & $1.499(16)$ \\
\hline $\mathrm{C}(30)-\mathrm{H}(30)$ & 0.9500 \\
\hline $\mathrm{C}(31)-\mathrm{F}(1 \mathrm{~A})$ & $1.274(17)$ \\
\hline $\mathrm{C}(31)-\mathrm{F}(2)$ & $1.315(14)$ \\
\hline $\mathrm{C}(31)-\mathrm{F}(3)$ & $1.338(13)$ \\
\hline $\mathrm{C}(31)-\mathrm{F}(1 \mathrm{~B})$ & $1.342(17)$ \\
\hline$C(32)-F(6)$ & $1.294(15)$ \\
\hline$C(32)-F(5)$ & $1.307(13)$ \\
\hline$C(32)-F(4)$ & $1.366(14)$ \\
\hline $\mathrm{C}(33)-\mathrm{C}(34)$ & $1.400(11)$ \\
\hline $\mathrm{C}(33)-\mathrm{C}(38)$ & $1.410(13)$ \\
\hline $\mathrm{C}(33)-\mathrm{B}(1)$ & $1.602(13)$ \\
\hline $\mathrm{C}(34)-\mathrm{C}(35)$ & $1.373(12)$ \\
\hline $\mathrm{C}(34)-\mathrm{H}(34)$ & 0.9500 \\
\hline$C(35)-C(36)$ & $1.387(13)$ \\
\hline $\mathrm{C}(35)-\mathrm{C}(39)$ & $1.475(13)$ \\
\hline $\mathrm{C}(36)-\mathrm{C}(37)$ & $1.371(13)$ \\
\hline $\mathrm{C}(36)-\mathrm{H}(36)$ & 0.9500 \\
\hline $\mathrm{C}(37)-\mathrm{C}(38)$ & $1.425(14)$ \\
\hline$C(37)-C(40)$ & $1.478(14)$ \\
\hline$C(39)-F(7)$ & $1.305(12)$ \\
\hline $\mathrm{C}(39)-\mathrm{F}(9)$ & $1.346(13)$ \\
\hline $\mathrm{C}(39)-\mathrm{F}(8)$ & $1.349(12)$ \\
\hline $\mathrm{C}(38)-\mathrm{H}(38)$ & 0.9500 \\
\hline $\mathrm{C}(40)-\mathrm{F}(12)$ & $1.317(12)$ \\
\hline $\mathrm{C}(40)-\mathrm{F}(10)$ & $1.336(12)$ \\
\hline $\mathrm{C}(40)-\mathrm{F}(11)$ & $1.345(11)$ \\
\hline $\mathrm{C}(41)-\mathrm{C}(46)$ & $1.385(13)$ \\
\hline $\mathrm{C}(41)-\mathrm{C}(42)$ & $1.411(12)$ \\
\hline $\mathrm{C}(41)-\mathrm{B}(1)$ & $1.655(13)$ \\
\hline $\mathrm{C}(42)-\mathrm{C}(43)$ & $1.374(13)$ \\
\hline $\mathrm{C}(42)-\mathrm{H}(42)$ & 0.9500 \\
\hline $\mathrm{C}(43)-\mathrm{C}(44)$ & $1.364(14)$ \\
\hline $\mathrm{C}(43)-\mathrm{C}(47)$ & $1.520(14)$ \\
\hline $\mathrm{C}(44)-\mathrm{C}(45)$ & $1.396(14)$ \\
\hline $\mathrm{C}(44)-\mathrm{H}(44)$ & 0.9500 \\
\hline $\mathrm{C}(45)-\mathrm{C}(46)$ & $1.381(13)$ \\
\hline $\mathrm{C}(45)-\mathrm{C}(48)$ & $1.476(15)$ \\
\hline $\mathrm{C}(46)-\mathrm{H}(46)$ & 0.9500 \\
\hline
\end{tabular}




\begin{tabular}{|c|c|}
\hline $\mathrm{C}(47)-\mathrm{F}(14)$ & $1.312(14)$ \\
\hline $\mathrm{C}(47)-\mathrm{F}(13)$ & $1.329(13)$ \\
\hline $\mathrm{C}(47)-\mathrm{F}(15)$ & $1.340(12)$ \\
\hline $\mathrm{C}(48)-\mathrm{F}(16)$ & $1.317(13)$ \\
\hline $\mathrm{C}(48)-\mathrm{F}(18)$ & $1.330(12)$ \\
\hline $\mathrm{C}(48)-\mathrm{F}(17)$ & $1.336(13)$ \\
\hline $\mathrm{C}(49)-\mathrm{C}(54)$ & $1.388(13)$ \\
\hline $\mathrm{C}(49)-\mathrm{C}(50)$ & $1.401(13)$ \\
\hline $\mathrm{C}(49)-\mathrm{B}(1)$ & $1.667(14)$ \\
\hline$C(50)-C(51)$ & $1.366(13)$ \\
\hline $\mathrm{C}(50)-\mathrm{H}(50)$ & 0.9500 \\
\hline $\mathrm{C}(51)-\mathrm{C}(52)$ & $1.415(13)$ \\
\hline $\mathrm{C}(51)-\mathrm{C}(55)$ & $1.446(14)$ \\
\hline $\mathrm{C}(52)-\mathrm{C}(53)$ & $1.376(14)$ \\
\hline $\mathrm{C}(52)-\mathrm{H}(52)$ & 0.9500 \\
\hline $\mathrm{C}(53)-\mathrm{C}(54)$ & $1.370(13)$ \\
\hline$C(53)-C(56)$ & $1.531(15)$ \\
\hline $\mathrm{C}(54)-\mathrm{H}(54)$ & 0.9500 \\
\hline $\mathrm{C}(55)-\mathrm{F}(24)$ & $1.306(12)$ \\
\hline $\mathrm{C}(55)-\mathrm{F}(22)$ & $1.316(13)$ \\
\hline $\mathrm{C}(55)-\mathrm{F}(23)$ & $1.374(14)$ \\
\hline $\mathrm{C}(56)-\mathrm{F}(20)$ & $1.300(16)$ \\
\hline $\mathrm{C}(56)-\mathrm{F}(21)$ & $1.331(13)$ \\
\hline $\mathrm{C}(56)-\mathrm{F}(19)$ & $1.332(13)$ \\
\hline $\mathrm{C}(57)-\mathrm{C}(58)$ & $1.340(19)$ \\
\hline $\mathrm{C}(57)-\mathrm{O}(5)$ & $1.358(15)$ \\
\hline $\mathrm{C}(57)-\mathrm{H}(57 \mathrm{~A})$ & 0.9900 \\
\hline $\mathrm{C}(57)-\mathrm{H}(57 \mathrm{~B})$ & 0.9900 \\
\hline $\mathrm{C}(58)-\mathrm{C}(59)$ & $1.45(2)$ \\
\hline $\mathrm{C}(58)-\mathrm{H}(58 \mathrm{~A})$ & 0.9900 \\
\hline $\mathrm{C}(58)-\mathrm{H}(58 \mathrm{~B})$ & 0.9900 \\
\hline $\mathrm{C}(59)-\mathrm{C}(60)$ & $1.52(2)$ \\
\hline $\mathrm{C}(59)-\mathrm{H}(59 \mathrm{~A})$ & 0.9900 \\
\hline $\mathrm{C}(59)-\mathrm{H}(59 \mathrm{~B})$ & 0.9900 \\
\hline $\mathrm{C}(60)-\mathrm{O}(5)$ & $1.532(19)$ \\
\hline $\mathrm{C}(60)-\mathrm{H}(60 \mathrm{~A})$ & 0.9900 \\
\hline $\mathrm{C}(60)-\mathrm{H}(60 \mathrm{~B})$ & 0.9900 \\
\hline $\mathrm{F}(1 \mathrm{~A})-\mathrm{F}(1 \mathrm{~B})$ & $0.958(17)$ \\
\hline $\mathrm{Fe}(1)-\operatorname{In}(1)$ & $2.4977(15)$ \\
\hline $\operatorname{Fe}(2)-\operatorname{In}(1)$ & $2.4935(16)$ \\
\hline $\mathrm{In}(1)-\mathrm{O}(5)$ & $2.382(8)$ \\
\hline$C(2)-C(1)-C(5)$ & $107.6(9)$ \\
\hline$C(2)-C(1)-C(6)$ & $124.6(10)$ \\
\hline$C(5)-C(1)-C(6)$ & $126.7(10)$ \\
\hline $\mathrm{C}(2)-\mathrm{C}(1)-\mathrm{Fe}(1)$ & 71.4(6) \\
\hline $\mathrm{C}(5)-\mathrm{C}(1)-\mathrm{Fe}(1)$ & $68.8(5)$ \\
\hline $\mathrm{C}(6)-\mathrm{C}(1)-\mathrm{Fe}(1)$ & $134.5(7)$ \\
\hline$C(1)-C(2)-C(3)$ & $106.8(9)$ \\
\hline$C(1)-C(2)-C(7)$ & $128.2(11)$ \\
\hline$C(3)-C(2)-C(7)$ & $124.9(12)$ \\
\hline $\mathrm{C}(1)-\mathrm{C}(2)-\mathrm{Fe}(1)$ & $69.9(6)$ \\
\hline $\mathrm{C}(3)-\mathrm{C}(2)-\mathrm{Fe}(1)$ & $68.7(6)$ \\
\hline $\mathrm{C}(7)-\mathrm{C}(2)-\mathrm{Fe}(1)$ & $128.6(9)$ \\
\hline$C(4)-C(3)-C(2)$ & 109.0(10) \\
\hline$C(4)-C(3)-C(8)$ & $127.0(12)$ \\
\hline$C(2)-C(3)-C(8)$ & $123.8(12)$ \\
\hline $\mathrm{C}(4)-\mathrm{C}(3)-\mathrm{Fe}(1)$ & $70.4(6)$ \\
\hline $\mathrm{C}(2)-\mathrm{C}(3)-\mathrm{Fe}(1)$ & $71.4(6)$ \\
\hline $\mathrm{C}(8)-\mathrm{C}(3)-\mathrm{Fe}(1)$ & $128.4(9)$ \\
\hline
\end{tabular}




\begin{tabular}{|c|c|}
\hline$C(3)-C(4)-C(5)$ & $108.5(10)$ \\
\hline$C(3)-C(4)-C(9)$ & $126.2(12)$ \\
\hline$C(5)-C(4)-C(9)$ & $125.2(12)$ \\
\hline $\mathrm{C}(3)-\mathrm{C}(4)-\mathrm{Fe}(1)$ & $71.2(6)$ \\
\hline $\mathrm{C}(5)-\mathrm{C}(4)-\mathrm{Fe}(1)$ & $70.0(6)$ \\
\hline $\mathrm{C}(9)-\mathrm{C}(4)-\mathrm{Fe}(1)$ & $128.7(8)$ \\
\hline$C(4)-C(5)-C(1)$ & 108.1(10) \\
\hline$C(4)-C(5)-C(10)$ & $126.6(11)$ \\
\hline$C(1)-C(5)-C(10)$ & 125.1(10) \\
\hline $\mathrm{C}(4)-\mathrm{C}(5)-\mathrm{Fe}(1)$ & $70.5(6)$ \\
\hline $\mathrm{C}(1)-\mathrm{C}(5)-\mathrm{Fe}(1)$ & $71.3(6)$ \\
\hline $\mathrm{C}(10)-\mathrm{C}(5)-\mathrm{Fe}(1)$ & $127.5(8)$ \\
\hline $\mathrm{C}(1)-\mathrm{C}(6)-\mathrm{H}(6 \mathrm{~A})$ & 109.5 \\
\hline $\mathrm{C}(1)-\mathrm{C}(6)-\mathrm{H}(6 \mathrm{~B})$ & 109.5 \\
\hline $\mathrm{H}(6 \mathrm{~A})-\mathrm{C}(6)-\mathrm{H}(6 \mathrm{~B})$ & 109.5 \\
\hline $\mathrm{C}(1)-\mathrm{C}(6)-\mathrm{H}(6 \mathrm{C})$ & 109.5 \\
\hline $\mathrm{H}(6 \mathrm{~A})-\mathrm{C}(6)-\mathrm{H}(6 \mathrm{C})$ & 109.5 \\
\hline $\mathrm{H}(6 \mathrm{~B})-\mathrm{C}(6)-\mathrm{H}(6 \mathrm{C})$ & 109.5 \\
\hline $\mathrm{C}(2)-\mathrm{C}(7)-\mathrm{H}(7 \mathrm{~A})$ & 109.5 \\
\hline $\mathrm{C}(2)-\mathrm{C}(7)-\mathrm{H}(7 \mathrm{~B})$ & 109.5 \\
\hline $\mathrm{H}(7 \mathrm{~A})-\mathrm{C}(7)-\mathrm{H}(7 \mathrm{~B})$ & 109.5 \\
\hline $\mathrm{C}(2)-\mathrm{C}(7)-\mathrm{H}(7 \mathrm{C})$ & 109.5 \\
\hline $\mathrm{H}(7 \mathrm{~A})-\mathrm{C}(7)-\mathrm{H}(7 \mathrm{C})$ & 109.5 \\
\hline $\mathrm{H}(7 \mathrm{~B})-\mathrm{C}(7)-\mathrm{H}(7 \mathrm{C})$ & 109.5 \\
\hline $\mathrm{C}(3)-\mathrm{C}(8)-\mathrm{H}(8 \mathrm{~A})$ & 109.5 \\
\hline $\mathrm{C}(3)-\mathrm{C}(8)-\mathrm{H}(8 \mathrm{~B})$ & 109.5 \\
\hline $\mathrm{H}(8 \mathrm{~A})-\mathrm{C}(8)-\mathrm{H}(8 \mathrm{~B})$ & 109.5 \\
\hline $\mathrm{C}(3)-\mathrm{C}(8)-\mathrm{H}(8 \mathrm{C})$ & 109.5 \\
\hline $\mathrm{H}(8 \mathrm{~A})-\mathrm{C}(8)-\mathrm{H}(8 \mathrm{C})$ & 109.5 \\
\hline $\mathrm{H}(8 \mathrm{~B})-\mathrm{C}(8)-\mathrm{H}(8 \mathrm{C})$ & 109.5 \\
\hline $\mathrm{C}(4)-\mathrm{C}(9)-\mathrm{H}(9 \mathrm{~A})$ & 109.5 \\
\hline $\mathrm{C}(4)-\mathrm{C}(9)-\mathrm{H}(9 \mathrm{~B})$ & 109.5 \\
\hline $\mathrm{H}(9 \mathrm{~A})-\mathrm{C}(9)-\mathrm{H}(9 \mathrm{~B})$ & 109.5 \\
\hline $\mathrm{C}(4)-\mathrm{C}(9)-\mathrm{H}(9 \mathrm{C})$ & 109.5 \\
\hline $\mathrm{H}(9 \mathrm{~A})-\mathrm{C}(9)-\mathrm{H}(9 \mathrm{C})$ & 109.5 \\
\hline $\mathrm{H}(9 \mathrm{~B})-\mathrm{C}(9)-\mathrm{H}(9 \mathrm{C})$ & 109.5 \\
\hline $\mathrm{C}(5)-\mathrm{C}(10)-\mathrm{H}(10 \mathrm{~A})$ & 109.5 \\
\hline $\mathrm{C}(5)-\mathrm{C}(10)-\mathrm{H}(10 \mathrm{~B})$ & 109.5 \\
\hline $\mathrm{H}(10 \mathrm{~A})-\mathrm{C}(10)-\mathrm{H}(10 \mathrm{~B})$ & 109.5 \\
\hline $\mathrm{C}(5)-\mathrm{C}(10)-\mathrm{H}(10 \mathrm{C})$ & 109.5 \\
\hline $\mathrm{H}(10 \mathrm{~A})-\mathrm{C}(10)-\mathrm{H}(10 \mathrm{C})$ & 109.5 \\
\hline $\mathrm{H}(10 \mathrm{~B})-\mathrm{C}(10)-\mathrm{H}(10 \mathrm{C})$ & 109.5 \\
\hline $\mathrm{C}(15)-\mathrm{C}(11)-\mathrm{C}(12)$ & 107.2(9) \\
\hline $\mathrm{C}(15)-\mathrm{C}(11)-\mathrm{C}(16)$ & $125.1(12)$ \\
\hline$C(12)-C(11)-C(16)$ & $127.5(12)$ \\
\hline $\mathrm{C}(15)-\mathrm{C}(11)-\mathrm{Fe}(2)$ & $70.5(6)$ \\
\hline $\mathrm{C}(12)-\mathrm{C}(11)-\mathrm{Fe}(2)$ & $70.0(6)$ \\
\hline $\mathrm{C}(16)-\mathrm{C}(11)-\mathrm{Fe}(2)$ & $128.5(9)$ \\
\hline $\mathrm{C}(13)-\mathrm{C}(12)-\mathrm{C}(11)$ & 109.9(9) \\
\hline $\mathrm{C}(13)-\mathrm{C}(12)-\mathrm{C}(17)$ & 124.1(11) \\
\hline$C(11)-C(12)-C(17)$ & $126.0(11)$ \\
\hline $\mathrm{C}(13)-\mathrm{C}(12)-\mathrm{Fe}(2)$ & $71.1(6)$ \\
\hline $\mathrm{C}(11)-\mathrm{C}(12)-\mathrm{Fe}(2)$ & $70.5(6)$ \\
\hline $\mathrm{C}(17)-\mathrm{C}(12)-\mathrm{Fe}(2)$ & $127.0(7)$ \\
\hline $\mathrm{C}(12)-\mathrm{C}(13)-\mathrm{C}(14)$ & $108.2(10)$ \\
\hline $\mathrm{C}(12)-\mathrm{C}(13)-\mathrm{C}(18)$ & $123.8(10)$ \\
\hline $\mathrm{C}(14)-\mathrm{C}(13)-\mathrm{C}(18)$ & $127.0(11)$ \\
\hline $\mathrm{C}(12)-\mathrm{C}(13)-\mathrm{Fe}(2)$ & $69.8(6)$ \\
\hline $\mathrm{C}(14)-\mathrm{C}(13)-\mathrm{Fe}(2)$ & $70.3(6)$ \\
\hline $\mathrm{C}(18)-\mathrm{C}(13)-\mathrm{Fe}(2)$ & $134.3(7)$ \\
\hline
\end{tabular}




\begin{tabular}{|c|c|}
\hline$C(13)-C(14)-C(15)$ & $106.3(10)$ \\
\hline$C(13)-C(14)-C(19)$ & $128.2(11)$ \\
\hline$C(15)-C(14)-C(19)$ & $125.4(11)$ \\
\hline $\mathrm{C}(13)-\mathrm{C}(14)-\mathrm{Fe}(2)$ & $70.6(6)$ \\
\hline $\mathrm{C}(15)-\mathrm{C}(14)-\mathrm{Fe}(2)$ & $69.2(6)$ \\
\hline $\mathrm{C}(19)-\mathrm{C}(14)-\mathrm{Fe}(2)$ & $127.5(9)$ \\
\hline$C(11)-C(15)-C(20)$ & $128.4(12)$ \\
\hline$C(11)-C(15)-C(14)$ & $108.4(10)$ \\
\hline$C(20)-C(15)-C(14)$ & $123.0(12)$ \\
\hline $\mathrm{C}(11)-\mathrm{C}(15)-\mathrm{Fe}(2)$ & $70.6(6)$ \\
\hline $\mathrm{C}(20)-\mathrm{C}(15)-\mathrm{Fe}(2)$ & $128.6(8)$ \\
\hline $\mathrm{C}(14)-\mathrm{C}(15)-\mathrm{Fe}(2)$ & $69.8(6)$ \\
\hline $\mathrm{C}(11)-\mathrm{C}(16)-\mathrm{H}(16 \mathrm{~A})$ & 109.5 \\
\hline $\mathrm{C}(11)-\mathrm{C}(16)-\mathrm{H}(16 \mathrm{~B})$ & 109.5 \\
\hline $\mathrm{H}(16 \mathrm{~A})-\mathrm{C}(16)-\mathrm{H}(16 \mathrm{~B})$ & 109.5 \\
\hline $\mathrm{C}(11)-\mathrm{C}(16)-\mathrm{H}(16 \mathrm{C})$ & 109.5 \\
\hline $\mathrm{H}(16 \mathrm{~A})-\mathrm{C}(16)-\mathrm{H}(16 \mathrm{C})$ & 109.5 \\
\hline $\mathrm{H}(16 \mathrm{~B})-\mathrm{C}(16)-\mathrm{H}(16 \mathrm{C})$ & 109.5 \\
\hline $\mathrm{C}(12)-\mathrm{C}(17)-\mathrm{H}(17 \mathrm{~A})$ & 109.5 \\
\hline $\mathrm{C}(12)-\mathrm{C}(17)-\mathrm{H}(17 \mathrm{~B})$ & 109.5 \\
\hline $\mathrm{H}(17 \mathrm{~A})-\mathrm{C}(17)-\mathrm{H}(17 \mathrm{~B})$ & 109.5 \\
\hline $\mathrm{C}(12)-\mathrm{C}(17)-\mathrm{H}(17 \mathrm{C})$ & 109.5 \\
\hline $\mathrm{H}(17 \mathrm{~A})-\mathrm{C}(17)-\mathrm{H}(17 \mathrm{C})$ & 109.5 \\
\hline $\mathrm{H}(17 \mathrm{~B})-\mathrm{C}(17)-\mathrm{H}(17 \mathrm{C})$ & 109.5 \\
\hline $\mathrm{C}(13)-\mathrm{C}(18)-\mathrm{H}(18 \mathrm{~A})$ & 109.5 \\
\hline $\mathrm{C}(13)-\mathrm{C}(18)-\mathrm{H}(18 \mathrm{~B})$ & 109.5 \\
\hline $\mathrm{H}(18 \mathrm{~A})-\mathrm{C}(18)-\mathrm{H}(18 \mathrm{~B})$ & 109.5 \\
\hline $\mathrm{C}(13)-\mathrm{C}(18)-\mathrm{H}(18 \mathrm{C})$ & 109.5 \\
\hline $\mathrm{H}(18 \mathrm{~A})-\mathrm{C}(18)-\mathrm{H}(18 \mathrm{C})$ & 109.5 \\
\hline $\mathrm{H}(18 \mathrm{~B})-\mathrm{C}(18)-\mathrm{H}(18 \mathrm{C})$ & 109.5 \\
\hline $\mathrm{C}(14)-\mathrm{C}(19)-\mathrm{H}(19 \mathrm{~A})$ & 109.5 \\
\hline $\mathrm{C}(14)-\mathrm{C}(19)-\mathrm{H}(19 \mathrm{~B})$ & 109.5 \\
\hline $\mathrm{H}(19 \mathrm{~A})-\mathrm{C}(19)-\mathrm{H}(19 \mathrm{~B})$ & 109.5 \\
\hline $\mathrm{C}(14)-\mathrm{C}(19)-\mathrm{H}(19 \mathrm{C})$ & 109.5 \\
\hline $\mathrm{H}(19 \mathrm{~A})-\mathrm{C}(19)-\mathrm{H}(19 \mathrm{C})$ & 109.5 \\
\hline $\mathrm{H}(19 \mathrm{~B})-\mathrm{C}(19)-\mathrm{H}(19 \mathrm{C})$ & 109.5 \\
\hline $\mathrm{C}(15)-\mathrm{C}(20)-\mathrm{H}(20 \mathrm{~A})$ & 109.5 \\
\hline $\mathrm{C}(15)-\mathrm{C}(20)-\mathrm{H}(20 \mathrm{~B})$ & 109.5 \\
\hline $\mathrm{H}(20 \mathrm{~A})-\mathrm{C}(20)-\mathrm{H}(20 \mathrm{~B})$ & 109.5 \\
\hline $\mathrm{C}(15)-\mathrm{C}(20)-\mathrm{H}(20 \mathrm{C})$ & 109.5 \\
\hline $\mathrm{H}(20 \mathrm{~A})-\mathrm{C}(20)-\mathrm{H}(20 \mathrm{C})$ & 109.5 \\
\hline $\mathrm{H}(20 \mathrm{~B})-\mathrm{C}(20)-\mathrm{H}(20 \mathrm{C})$ & 109.5 \\
\hline $\mathrm{O}(1)-\mathrm{C}(21)-\mathrm{Fe}(2)$ & $175.3(12)$ \\
\hline $\mathrm{O}(2)-\mathrm{C}(22)-\mathrm{Fe}(2)$ & $177.5(11)$ \\
\hline $\mathrm{O}(3)-\mathrm{C}(23)-\mathrm{Fe}(1)$ & $177.2(12)$ \\
\hline $\mathrm{O}(4)-\mathrm{C}(24)-\mathrm{Fe}(1)$ & 174.1(11) \\
\hline$C(26)-C(25)-C(30)$ & 117.1(9) \\
\hline $\mathrm{C}(26)-\mathrm{C}(25)-\mathrm{B}(1)$ & 121.6(8) \\
\hline $\mathrm{C}(30)-\mathrm{C}(25)-\mathrm{B}(1)$ & $121.0(8)$ \\
\hline$C(25)-C(26)-C(27)$ & 120.3(9) \\
\hline $\mathrm{C}(25)-\mathrm{C}(26)-\mathrm{H}(26)$ & 119.8 \\
\hline $\mathrm{C}(27)-\mathrm{C}(26)-\mathrm{H}(26)$ & 119.8 \\
\hline $\mathrm{C}(28)-\mathrm{C}(27)-\mathrm{C}(26)$ & 121.0(8) \\
\hline$C(28)-C(27)-C(31)$ & $120.1(8)$ \\
\hline$C(26)-C(27)-C(31)$ & 118.9(9) \\
\hline $\mathrm{C}(27)-\mathrm{C}(28)-\mathrm{C}(29)$ & $118.9(8)$ \\
\hline $\mathrm{C}(27)-\mathrm{C}(28)-\mathrm{H}(28)$ & 120.6 \\
\hline $\mathrm{C}(29)-\mathrm{C}(28)-\mathrm{H}(28)$ & 120.6 \\
\hline$C(30)-C(29)-C(28)$ & 121.2(9) \\
\hline$C(30)-C(29)-C(32)$ & $118.4(9)$ \\
\hline
\end{tabular}




\begin{tabular}{|c|c|}
\hline $\mathrm{C}(28)-\mathrm{C}(29)-\mathrm{C}(32)$ & $120.3(9)$ \\
\hline $\mathrm{C}(29)-\mathrm{C}(30)-\mathrm{C}(25)$ & $121.4(10)$ \\
\hline $\mathrm{C}(29)-\mathrm{C}(30)-\mathrm{H}(30)$ & 119.3 \\
\hline $\mathrm{C}(25)-\mathrm{C}(30)-\mathrm{H}(30)$ & 119.3 \\
\hline $\mathrm{F}(1 \mathrm{~A})-\mathrm{C}(31)-\mathrm{F}(2)$ & $127.5(13)$ \\
\hline $\mathrm{F}(1 \mathrm{~A})-\mathrm{C}(31)-\mathrm{F}(3)$ & $88.9(10)$ \\
\hline $\mathrm{F}(2)-\mathrm{C}(31)-\mathrm{F}(3)$ & $97.2(9)$ \\
\hline $\mathrm{F}(1 \mathrm{~A})-\mathrm{C}(31)-\mathrm{F}(1 \mathrm{~B})$ & $42.9(8)$ \\
\hline $\mathrm{F}(2)-\mathrm{C}(31)-\mathrm{F}(1 \mathrm{~B})$ & $92.9(11)$ \\
\hline $\mathrm{F}(3)-\mathrm{C}(31)-\mathrm{F}(1 \mathrm{~B})$ & $120.9(11)$ \\
\hline $\mathrm{F}(1 \mathrm{~A})-\mathrm{C}(31)-\mathrm{C}(27)$ & $112.7(11)$ \\
\hline $\mathrm{F}(2)-\mathrm{C}(31)-\mathrm{C}(27)$ & 112.3(9) \\
\hline $\mathrm{F}(3)-\mathrm{C}(31)-\mathrm{C}(27)$ & $113.0(10)$ \\
\hline $\mathrm{F}(1 \mathrm{~B})-\mathrm{C}(31)-\mathrm{C}(27)$ & $116.0(10)$ \\
\hline $\mathrm{F}(6)-\mathrm{C}(32)-\mathrm{F}(5)$ & $107.3(10)$ \\
\hline $\mathrm{F}(6)-\mathrm{C}(32)-\mathrm{F}(4)$ & 106.1(11) \\
\hline $\mathrm{F}(5)-\mathrm{C}(32)-\mathrm{F}(4)$ & 101.9(10) \\
\hline$F(6)-C(32)-C(29)$ & 116.7(9) \\
\hline $\mathrm{F}(5)-\mathrm{C}(32)-\mathrm{C}(29)$ & $112.4(11)$ \\
\hline $\mathrm{F}(4)-\mathrm{C}(32)-\mathrm{C}(29)$ & 111.3(10) \\
\hline $\mathrm{C}(34)-\mathrm{C}(33)-\mathrm{C}(38)$ & 114.1(8) \\
\hline $\mathrm{C}(34)-\mathrm{C}(33)-\mathrm{B}(1)$ & 124.1(8) \\
\hline $\mathrm{C}(38)-\mathrm{C}(33)-\mathrm{B}(1)$ & $121.7(8)$ \\
\hline$C(35)-C(34)-C(33)$ & $124.4(9)$ \\
\hline $\mathrm{C}(35)-\mathrm{C}(34)-\mathrm{H}(34)$ & 117.8 \\
\hline $\mathrm{C}(33)-\mathrm{C}(34)-\mathrm{H}(34)$ & 117.8 \\
\hline $\mathrm{C}(34)-\mathrm{C}(35)-\mathrm{C}(36)$ & 120.1(9) \\
\hline C(34)-C(35)-C(39) & 118.1(9) \\
\hline$C(36)-C(35)-C(39)$ & $121.7(8)$ \\
\hline$C(37)-C(36)-C(35)$ & 119.1(9) \\
\hline $\mathrm{C}(37)-\mathrm{C}(36)-\mathrm{H}(36)$ & 120.4 \\
\hline $\mathrm{C}(35)-\mathrm{C}(36)-\mathrm{H}(36)$ & 120.4 \\
\hline $\mathrm{C}(36)-\mathrm{C}(37)-\mathrm{C}(38)$ & $119.9(9)$ \\
\hline$C(36)-C(37)-C(40)$ & $122.8(9)$ \\
\hline $\mathrm{C}(38)-\mathrm{C}(37)-\mathrm{C}(40)$ & $117.2(9)$ \\
\hline $\mathrm{F}(7)-\mathrm{C}(39)-\mathrm{F}(9)$ & $105.4(9)$ \\
\hline $\mathrm{F}(7)-\mathrm{C}(39)-\mathrm{F}(8)$ & 106.3(9) \\
\hline $\mathrm{F}(9)-\mathrm{C}(39)-\mathrm{F}(8)$ & $103.9(8)$ \\
\hline$F(7)-C(39)-C(35)$ & $115.0(9)$ \\
\hline $\mathrm{F}(9)-\mathrm{C}(39)-\mathrm{C}(35)$ & $112.6(9)$ \\
\hline $\mathrm{F}(8)-\mathrm{C}(39)-\mathrm{C}(35)$ & $112.8(9)$ \\
\hline $\mathrm{C}(33)-\mathrm{C}(38)-\mathrm{C}(37)$ & 122.2(9) \\
\hline $\mathrm{C}(33)-\mathrm{C}(38)-\mathrm{H}(38)$ & 118.9 \\
\hline $\mathrm{C}(37)-\mathrm{C}(38)-\mathrm{H}(38)$ & 118.9 \\
\hline $\mathrm{F}(12)-\mathrm{C}(40)-\mathrm{F}(10)$ & $106.4(9)$ \\
\hline $\mathrm{F}(12)-\mathrm{C}(40)-\mathrm{F}(11)$ & 106.7(8) \\
\hline $\mathrm{F}(10)-\mathrm{C}(40)-\mathrm{F}(11)$ & $105.3(8)$ \\
\hline $\mathrm{F}(12)-\mathrm{C}(40)-\mathrm{C}(37)$ & $113.7(9)$ \\
\hline $\mathrm{F}(10)-\mathrm{C}(40)-\mathrm{C}(37)$ & $112.2(8)$ \\
\hline $\mathrm{F}(11)-\mathrm{C}(40)-\mathrm{C}(37)$ & $112.0(9)$ \\
\hline$C(46)-C(41)-C(42)$ & $114.2(8)$ \\
\hline $\mathrm{C}(46)-\mathrm{C}(41)-\mathrm{B}(1)$ & $124.5(8)$ \\
\hline $\mathrm{C}(42)-\mathrm{C}(41)-\mathrm{B}(1)$ & 121.2(8) \\
\hline $\mathrm{C}(43)-\mathrm{C}(42)-\mathrm{C}(41)$ & 123.2(9) \\
\hline $\mathrm{C}(43)-\mathrm{C}(42)-\mathrm{H}(42)$ & 118.4 \\
\hline $\mathrm{C}(41)-\mathrm{C}(42)-\mathrm{H}(42)$ & 118.4 \\
\hline $\mathrm{C}(44)-\mathrm{C}(43)-\mathrm{C}(42)$ & $121.3(9)$ \\
\hline $\mathrm{C}(44)-\mathrm{C}(43)-\mathrm{C}(47)$ & $120.7(9)$ \\
\hline $\mathrm{C}(42)-\mathrm{C}(43)-\mathrm{C}(47)$ & $118.0(10)$ \\
\hline $\mathrm{C}(43)-\mathrm{C}(44)-\mathrm{C}(45)$ & $117.2(9)$ \\
\hline
\end{tabular}




\begin{tabular}{|c|c|}
\hline $\mathrm{C}(43)-\mathrm{C}(44)-\mathrm{H}(44)$ & 121.4 \\
\hline $\mathrm{C}(45)-\mathrm{C}(44)-\mathrm{H}(44)$ & 121.4 \\
\hline$C(46)-C(45)-C(44)$ & 121.2(9) \\
\hline $\mathrm{C}(46)-\mathrm{C}(45)-\mathrm{C}(48)$ & 119.1(9) \\
\hline $\mathrm{C}(44)-\mathrm{C}(45)-\mathrm{C}(48)$ & $119.6(9)$ \\
\hline$C(45)-C(46)-C(41)$ & $122.8(9)$ \\
\hline $\mathrm{C}(45)-\mathrm{C}(46)-\mathrm{H}(46)$ & 118.6 \\
\hline $\mathrm{C}(41)-\mathrm{C}(46)-\mathrm{H}(46)$ & 118.6 \\
\hline $\mathrm{F}(14)-\mathrm{C}(47)-\mathrm{F}(13)$ & $109.8(11)$ \\
\hline $\mathrm{F}(14)-\mathrm{C}(47)-\mathrm{F}(15)$ & 107.7(9) \\
\hline $\mathrm{F}(13)-\mathrm{C}(47)-\mathrm{F}(15)$ & $105.5(9)$ \\
\hline $\mathrm{F}(14)-\mathrm{C}(47)-\mathrm{C}(43)$ & $113.5(9)$ \\
\hline $\mathrm{F}(13)-\mathrm{C}(47)-\mathrm{C}(43)$ & 108.7(9) \\
\hline $\mathrm{F}(15)-\mathrm{C}(47)-\mathrm{C}(43)$ & $111.2(10)$ \\
\hline $\mathrm{F}(16)-\mathrm{C}(48)-\mathrm{F}(18)$ & $105.3(10)$ \\
\hline $\mathrm{F}(16)-\mathrm{C}(48)-\mathrm{F}(17)$ & $105.6(9)$ \\
\hline $\mathrm{F}(18)-\mathrm{C}(48)-\mathrm{F}(17)$ & $103.7(10)$ \\
\hline $\mathrm{F}(16)-\mathrm{C}(48)-\mathrm{C}(45)$ & $114.9(10)$ \\
\hline $\mathrm{F}(18)-\mathrm{C}(48)-\mathrm{C}(45)$ & $113.5(9)$ \\
\hline $\mathrm{F}(17)-\mathrm{C}(48)-\mathrm{C}(45)$ & $112.8(9)$ \\
\hline$C(54)-C(49)-C(50)$ & $115.4(9)$ \\
\hline $\mathrm{C}(54)-\mathrm{C}(49)-\mathrm{B}(1)$ & $122.3(8)$ \\
\hline $\mathrm{C}(50)-\mathrm{C}(49)-\mathrm{B}(1)$ & $122.1(8)$ \\
\hline$C(51)-C(50)-C(49)$ & $123.5(9)$ \\
\hline $\mathrm{C}(51)-\mathrm{C}(50)-\mathrm{H}(50)$ & 118.3 \\
\hline $\mathrm{C}(49)-\mathrm{C}(50)-\mathrm{H}(50)$ & 118.3 \\
\hline$C(50)-C(51)-C(52)$ & $119.4(9)$ \\
\hline$C(50)-C(51)-C(55)$ & 119.2(9) \\
\hline $\mathrm{C}(52)-\mathrm{C}(51)-\mathrm{C}(55)$ & $121.5(9)$ \\
\hline$C(53)-C(52)-C(51)$ & $117.9(9)$ \\
\hline $\mathrm{C}(53)-\mathrm{C}(52)-\mathrm{H}(52)$ & 121.1 \\
\hline $\mathrm{C}(51)-\mathrm{C}(52)-\mathrm{H}(52)$ & 121.1 \\
\hline$C(54)-C(53)-C(52)$ & $121.4(9)$ \\
\hline $\mathrm{C}(54)-\mathrm{C}(53)-\mathrm{C}(56)$ & 119.2(9) \\
\hline $\mathrm{C}(52)-\mathrm{C}(53)-\mathrm{C}(56)$ & $119.4(9)$ \\
\hline $\mathrm{C}(53)-\mathrm{C}(54)-\mathrm{C}(49)$ & $122.5(9)$ \\
\hline $\mathrm{C}(53)-\mathrm{C}(54)-\mathrm{H}(54)$ & 118.7 \\
\hline $\mathrm{C}(49)-\mathrm{C}(54)-\mathrm{H}(54)$ & 118.7 \\
\hline $\mathrm{F}(24)-\mathrm{C}(55)-\mathrm{F}(22)$ & $105.3(9)$ \\
\hline $\mathrm{F}(24)-\mathrm{C}(55)-\mathrm{F}(23)$ & $99.8(10)$ \\
\hline $\mathrm{F}(22)-\mathrm{C}(55)-\mathrm{F}(23)$ & 104.3(9) \\
\hline $\mathrm{F}(24)-\mathrm{C}(55)-\mathrm{C}(51)$ & $116.2(9)$ \\
\hline $\mathrm{F}(22)-\mathrm{C}(55)-\mathrm{C}(51)$ & $116.3(10)$ \\
\hline $\mathrm{F}(23)-\mathrm{C}(55)-\mathrm{C}(51)$ & $113.1(8)$ \\
\hline $\mathrm{F}(20)-\mathrm{C}(56)-\mathrm{F}(21)$ & $106.7(10)$ \\
\hline $\mathrm{F}(20)-\mathrm{C}(56)-\mathrm{F}(19)$ & $107.2(11)$ \\
\hline $\mathrm{F}(21)-\mathrm{C}(56)-\mathrm{F}(19)$ & $107.8(10)$ \\
\hline$F(20)-C(56)-C(53)$ & $112.4(10)$ \\
\hline $\mathrm{F}(21)-\mathrm{C}(56)-\mathrm{C}(53)$ & $109.7(11)$ \\
\hline $\mathrm{F}(19)-\mathrm{C}(56)-\mathrm{C}(53)$ & $112.7(10)$ \\
\hline $\mathrm{C}(58)-\mathrm{C}(57)-\mathrm{O}(5)$ & $114.6(13)$ \\
\hline $\mathrm{C}(58)-\mathrm{C}(57)-\mathrm{H}(57 \mathrm{~A})$ & 108.6 \\
\hline $\mathrm{O}(5)-\mathrm{C}(57)-\mathrm{H}(57 \mathrm{~A})$ & 108.6 \\
\hline $\mathrm{C}(58)-\mathrm{C}(57)-\mathrm{H}(57 \mathrm{~B})$ & 108.6 \\
\hline $\mathrm{O}(5)-\mathrm{C}(57)-\mathrm{H}(57 \mathrm{~B})$ & 108.6 \\
\hline $\mathrm{H}(57 \mathrm{~A})-\mathrm{C}(57)-\mathrm{H}(57 \mathrm{~B})$ & 107.6 \\
\hline$C(57)-C(58)-C(59)$ & $104.4(14)$ \\
\hline $\mathrm{C}(57)-\mathrm{C}(58)-\mathrm{H}(58 \mathrm{~A})$ & 110.9 \\
\hline $\mathrm{C}(59)-\mathrm{C}(58)-\mathrm{H}(58 \mathrm{~A})$ & 110.9 \\
\hline $\mathrm{C}(57)-\mathrm{C}(58)-\mathrm{H}(58 \mathrm{~B})$ & 110.9 \\
\hline
\end{tabular}




\begin{tabular}{|c|c|}
\hline $\mathrm{C}(59)-\mathrm{C}(58)-\mathrm{H}(58 \mathrm{~B})$ & 110.9 \\
\hline $\mathrm{H}(58 \mathrm{~A})-\mathrm{C}(58)-\mathrm{H}(58 \mathrm{~B})$ & 108.9 \\
\hline$C(58)-C(59)-C(60)$ & $112.5(13)$ \\
\hline $\mathrm{C}(58)-\mathrm{C}(59)-\mathrm{H}(59 \mathrm{~A})$ & 109.1 \\
\hline $\mathrm{C}(60)-\mathrm{C}(59)-\mathrm{H}(59 \mathrm{~A})$ & 109.1 \\
\hline $\mathrm{C}(58)-\mathrm{C}(59)-\mathrm{H}(59 \mathrm{~B})$ & 109.1 \\
\hline $\mathrm{C}(60)-\mathrm{C}(59)-\mathrm{H}(59 \mathrm{~B})$ & 109.1 \\
\hline $\mathrm{H}(59 \mathrm{~A})-\mathrm{C}(59)-\mathrm{H}(59 \mathrm{~B})$ & 107.8 \\
\hline $\mathrm{C}(59)-\mathrm{C}(60)-\mathrm{O}(5)$ & $96.7(12)$ \\
\hline $\mathrm{C}(59)-\mathrm{C}(60)-\mathrm{H}(60 \mathrm{~A})$ & 112.4 \\
\hline $\mathrm{O}(5)-\mathrm{C}(60)-\mathrm{H}(60 \mathrm{~A})$ & 112.4 \\
\hline $\mathrm{C}(59)-\mathrm{C}(60)-\mathrm{H}(60 \mathrm{~B})$ & 112.4 \\
\hline $\mathrm{O}(5)-\mathrm{C}(60)-\mathrm{H}(60 \mathrm{~B})$ & 112.4 \\
\hline$H(60 A)-C(60)-H(60 B)$ & 110.0 \\
\hline $\mathrm{C}(33)-\mathrm{B}(1)-\mathrm{C}(25)$ & $105.1(7)$ \\
\hline $\mathrm{C}(33)-\mathrm{B}(1)-\mathrm{C}(41)$ & $112.8(7)$ \\
\hline $\mathrm{C}(25)-\mathrm{B}(1)-\mathrm{C}(41)$ & $111.6(7)$ \\
\hline $\mathrm{C}(33)-\mathrm{B}(1)-\mathrm{C}(49)$ & $112.4(7)$ \\
\hline $\mathrm{C}(25)-\mathrm{B}(1)-\mathrm{C}(49)$ & $112.0(7)$ \\
\hline $\mathrm{C}(41)-\mathrm{B}(1)-\mathrm{C}(49)$ & $103.0(7)$ \\
\hline $\mathrm{F}(1 \mathrm{~B})-\mathrm{F}(1 \mathrm{~A})-\mathrm{C}(31)$ & 72.3(14) \\
\hline $\mathrm{F}(1 \mathrm{~A})-\mathrm{F}(1 \mathrm{~B})-\mathrm{C}(31)$ & $64.8(13)$ \\
\hline $\mathrm{C}(24)-\mathrm{Fe}(1)-\mathrm{C}(23)$ & $94.7(6)$ \\
\hline $\mathrm{C}(24)-\mathrm{Fe}(1)-\mathrm{C}(5)$ & $97.1(5)$ \\
\hline $\mathrm{C}(23)-\mathrm{Fe}(1)-\mathrm{C}(5)$ & $162.7(5)$ \\
\hline $\mathrm{C}(24)-\mathrm{Fe}(1)-\mathrm{C}(4)$ & $91.6(5)$ \\
\hline $\mathrm{C}(23)-\mathrm{Fe}(1)-\mathrm{C}(4)$ & $127.8(5)$ \\
\hline $\mathrm{C}(5)-\mathrm{Fe}(1)-\mathrm{C}(4)$ & $39.5(4)$ \\
\hline $\mathrm{C}(24)-\mathrm{Fe}(1)-\mathrm{C}(3)$ & $120.7(5)$ \\
\hline $\mathrm{C}(23)-\mathrm{Fe}(1)-\mathrm{C}(3)$ & $97.6(5)$ \\
\hline $\mathrm{C}(5)-\mathrm{Fe}(1)-\mathrm{C}(3)$ & $65.5(5)$ \\
\hline $\mathrm{C}(4)-\mathrm{Fe}(1)-\mathrm{C}(3)$ & $38.4(5)$ \\
\hline $\mathrm{C}(24)-\mathrm{Fe}(1)-\mathrm{C}(1)$ & $133.4(5)$ \\
\hline $\mathrm{C}(23)-\mathrm{Fe}(1)-\mathrm{C}(1)$ & $131.6(5)$ \\
\hline $\mathrm{C}(5)-\mathrm{Fe}(1)-\mathrm{C}(1)$ & 39.9(4) \\
\hline $\mathrm{C}(4)-\mathrm{Fe}(1)-\mathrm{C}(1)$ & $66.3(4)$ \\
\hline $\mathrm{C}(3)-\mathrm{Fe}(1)-\mathrm{C}(1)$ & $65.9(4)$ \\
\hline $\mathrm{C}(24)-\mathrm{Fe}(1)-\mathrm{C}(2)$ & $157.5(5)$ \\
\hline $\mathrm{C}(23)-\mathrm{Fe}(1)-\mathrm{C}(2)$ & $99.2(5)$ \\
\hline $\mathrm{C}(5)-\mathrm{Fe}(1)-\mathrm{C}(2)$ & $65.9(4)$ \\
\hline $\mathrm{C}(4)-\mathrm{Fe}(1)-\mathrm{C}(2)$ & $65.8(4)$ \\
\hline $\mathrm{C}(3)-\mathrm{Fe}(1)-\mathrm{C}(2)$ & $39.9(5)$ \\
\hline $\mathrm{C}(1)-\mathrm{Fe}(1)-\mathrm{C}(2)$ & $38.7(4)$ \\
\hline $\mathrm{C}(24)-\mathrm{Fe}(1)-\operatorname{In}(1)$ & $88.2(4)$ \\
\hline $\mathrm{C}(23)-\mathrm{Fe}(1)-\operatorname{In}(1)$ & $91.0(4)$ \\
\hline $\mathrm{C}(5)-\mathrm{Fe}(1)-\operatorname{In}(1)$ & 101.9(3) \\
\hline $\mathrm{C}(4)-\mathrm{Fe}(1)-\operatorname{In}(1)$ & 141.1(3) \\
\hline $\mathrm{C}(3)-\mathrm{Fe}(1)-\operatorname{In}(1)$ & $148.7(3)$ \\
\hline $\mathrm{C}(1)-\mathrm{Fe}(1)-\operatorname{In}(1)$ & $86.0(3)$ \\
\hline $\mathrm{C}(2)-\mathrm{Fe}(1)-\operatorname{In}(1)$ & 109.1(3) \\
\hline $\mathrm{C}(21)-\mathrm{Fe}(2)-\mathrm{C}(22)$ & $95.3(6)$ \\
\hline$C(21)-F e(2)-C(12)$ & $95.1(5)$ \\
\hline$C(22)-F e(2)-C(12)$ & $158.9(5)$ \\
\hline $\mathrm{C}(21)-\mathrm{Fe}(2)-\mathrm{C}(15)$ & $125.2(6)$ \\
\hline$C(22)-F e(2)-C(15)$ & $93.6(5)$ \\
\hline$C(12)-F e(2)-C(15)$ & $65.5(4)$ \\
\hline$C(21)-F e(2)-C(11)$ & $93.2(5)$ \\
\hline $\mathrm{C}(22)-\mathrm{Fe}(2)-\mathrm{C}(11)$ & $121.5(5)$ \\
\hline $\mathrm{C}(12)-\mathrm{Fe}(2)-\mathrm{C}(11)$ & $39.5(4)$ \\
\hline $\mathrm{C}(15)-\mathrm{Fe}(2)-\mathrm{C}(11)$ & $38.9(5)$ \\
\hline
\end{tabular}




$\begin{array}{lr}\mathrm{C}(21)-\mathrm{Fe}(2)-\mathrm{C}(14) & 159.4(5) \\ \mathrm{C}(22)-\mathrm{Fe}(2)-\mathrm{C}(14) & 100.5(5) \\ \mathrm{C}(12)-\mathrm{Fe}(2)-\mathrm{C}(14) & 65.9(4) \\ \mathrm{C}(15)-\mathrm{Fe}(2)-\mathrm{C}(14) & 41.0(5) \\ \mathrm{C}(11)-\mathrm{Fe}(2)-\mathrm{C}(14) & 67.3(5) \\ \mathrm{C}(21)-\mathrm{Fe}(2)-\mathrm{C}(13) & 128.2(5) \\ \mathrm{C}(22)-\mathrm{Fe}(2)-\mathrm{C}(13) & 136.3(5) \\ \mathrm{C}(12)-\mathrm{Fe}(2)-\mathrm{C}(13) & 39.1(4) \\ \mathrm{C}(15)-\mathrm{Fe}(2)-\mathrm{C}(13) & 66.5(4) \\ \mathrm{C}(11)-\mathrm{Fe}(2)-\mathrm{C}(13) & 66.6(4) \\ \mathrm{C}(14)-\mathrm{Fe}(2)-\mathrm{C}(13) & 39.1(4) \\ \mathrm{C}(21)-\mathrm{Fe}(2)-\mathrm{In}(1) & 92.3(4) \\ \mathrm{C}(22)-\mathrm{Fe}(2)-\mathrm{In}(1) & 87.6(4) \\ \mathrm{C}(12)-\mathrm{Fe}(2)-\mathrm{In}(1) & 110.3(3) \\ \mathrm{C}(15)-\mathrm{Fe}(2)-\mathrm{In}(1) & 142.1(4) \\ \mathrm{C}(11)-\mathrm{Fe}(2)-\mathrm{In}(1) & 149.7(3) \\ \mathrm{C}(14)-\mathrm{Fe}(2)-\operatorname{In}(1) & 101.5(4) \\ \mathrm{C}(13)-\mathrm{Fe}(2)-\mathrm{In}(1) & 86.8(3) \\ \mathrm{O}(5)-\mathrm{In}(1)-\mathrm{Fe}(2) & 103.2(3) \\ \mathrm{O}(5)-\operatorname{In}(1)-\mathrm{Fe}(1) & 100.1(3) \\ \mathrm{Fe}(2)-\operatorname{In}(1)-\mathrm{Fe}(1) & 156.72(6) \\ \mathrm{C}(57)-\mathrm{O}(5)-\mathrm{C}(60) & 110.8(10) \\ \mathrm{C}(57)-\mathrm{O}(5)-\operatorname{In}(1) & 134.4(8) \\ \mathrm{C}(60)-\mathrm{O}(5)-\operatorname{In}(1) & 114.7(8)\end{array}$

Symmetry transformations used to generate equivalent atoms: 
Table 4.4 Anisotropic displacement parameters $\left(\AA^{2} \mathrm{x} 10^{3}\right)$ for $\mathbf{1 4}$.

The anisotropic displacement factor exponent takes the form: $-2 \pi^{2}\left[h^{2} a^{* 2} U^{11}+\ldots+2 h k a^{*} b^{*} U^{12}\right]$

\begin{tabular}{|c|c|c|c|c|c|c|}
\hline & $\mathrm{U}^{11}$ & $\mathrm{U}^{22}$ & $\mathrm{U}^{33}$ & $\mathrm{U}^{23}$ & $\mathrm{U}^{13}$ & $\mathrm{U}^{12}$ \\
\hline $\mathrm{C}(1)$ & $39(5)$ & $23(4)$ & $47(5)$ & $2(4)$ & $8(4)$ & $0(4)$ \\
\hline$C(2)$ & $58(7)$ & $30(5)$ & $66(7)$ & $-11(5)$ & $-9(6)$ & $3(6)$ \\
\hline $\mathrm{C}(3)$ & $40(6)$ & $38(5)$ & $90(9)$ & $5(7)$ & $-20(7)$ & $-10(5)$ \\
\hline $\mathrm{C}(4)$ & $45(7)$ & $30(5)$ & $76(8)$ & $5(5)$ & $14(6)$ & $-2(5)$ \\
\hline$C(5)$ & $39(6)$ & $29(5)$ & $63(7)$ & $16(5)$ & $13(5)$ & $-7(5)$ \\
\hline$C(6)$ & $36(6)$ & $35(5)$ & $85(8)$ & $6(5)$ & $5(6)$ & $-3(5)$ \\
\hline$C(7)$ & 121(13) & $59(8)$ & $59(8)$ & $-20(6)$ & $-6(8)$ & $-14(8)$ \\
\hline $\mathrm{C}(8)$ & $95(5)$ & $95(5)$ & $96(5)$ & $0(1)$ & $-1(1)$ & $0(1)$ \\
\hline $\mathrm{C}(9)$ & $78(4)$ & $79(4)$ & $79(4)$ & $0(1)$ & $1(1)$ & $0(1)$ \\
\hline$C(10)$ & $93(10)$ & $55(7)$ & $55(7)$ & $-4(6)$ & $9(7)$ & $2(8)$ \\
\hline$C(11)$ & $28(6)$ & $41(6)$ & $77(8)$ & $-8(6)$ & $5(5)$ & $-12(5)$ \\
\hline$C(12)$ & $50(7)$ & $34(5)$ & $41(6)$ & $-3(4)$ & $-5(5)$ & $3(5)$ \\
\hline$C(13)$ & $43(6)$ & $31(5)$ & $50(6)$ & $-5(5)$ & $-9(5)$ & $-3(5)$ \\
\hline $\mathrm{C}(14)$ & $64(8)$ & $38(6)$ & $43(6)$ & $-3(5)$ & $3(5)$ & $11(5)$ \\
\hline$C(15)$ & $60(8)$ & $29(5)$ & $67(8)$ & $-18(5)$ & $-22(6)$ & $5(5)$ \\
\hline$C(16)$ & $87(5)$ & $87(5)$ & $87(5)$ & $0(1)$ & $0(1)$ & $0(1)$ \\
\hline$C(17)$ & $138(14)$ & $57(8)$ & $47(7)$ & $-7(6)$ & $17(8)$ & $-46(9)$ \\
\hline $\mathrm{C}(18)$ & $50(7)$ & $33(5)$ & 111(10) & $-10(6)$ & $-27(8)$ & $10(6)$ \\
\hline$C(19)$ & $76(4)$ & $75(4)$ & $76(4)$ & $0(1)$ & $1(1)$ & $0(1)$ \\
\hline$C(20)$ & $77(4)$ & $77(4)$ & $77(4)$ & $0(1)$ & $-1(1)$ & $0(1)$ \\
\hline $\mathrm{C}(21)$ & $50(7)$ & $67(8)$ & $67(8)$ & $-19(6)$ & $31(6)$ & $-14(6)$ \\
\hline $\mathrm{C}(22)$ & $44(7)$ & $35(6)$ & $73(8)$ & $4(6)$ & $-18(6)$ & $6(5)$ \\
\hline$C(23)$ & $50(7)$ & $58(7)$ & $62(7)$ & $2(7)$ & $-9(6)$ & $-1(6)$ \\
\hline $\mathrm{C}(24)$ & $36(6)$ & $50(7)$ & $67(8)$ & $-1(6)$ & $11(6)$ & $-14(6)$ \\
\hline $\mathrm{C}(25)$ & $42(6)$ & $29(4)$ & $28(5)$ & $-3(4)$ & $-2(4)$ & $9(4)$ \\
\hline$C(26)$ & $35(6)$ & $32(5)$ & $32(5)$ & $0(4)$ & $1(4)$ & $11(4)$ \\
\hline$C(27)$ & $31(5)$ & $53(6)$ & $22(4)$ & $5(4)$ & $3(4)$ & $3(5)$ \\
\hline $\mathrm{C}(28)$ & $35(5)$ & $44(6)$ & $25(5)$ & $13(4)$ & $6(4)$ & $-1(4)$ \\
\hline $\mathrm{C}(29)$ & $41(6)$ & $29(5)$ & $34(5)$ & $1(4)$ & $3(4)$ & 1(4) \\
\hline $\mathrm{C}(30)$ & $37(6)$ & $41(6)$ & $40(6)$ & $-3(5)$ & $4(5)$ & $3(5)$ \\
\hline $\mathrm{C}(31)$ & $47(6)$ & $39(5)$ & $47(6)$ & $12(4)$ & $16(5)$ & $4(5)$ \\
\hline$C(32)$ & $45(7)$ & $45(7)$ & $74(8)$ & $1(6)$ & $30(7)$ & $-2(6)$ \\
\hline $\mathrm{C}(33)$ & $23(4)$ & $32(5)$ & $22(4)$ & $-1(4)$ & $-9(3)$ & $-8(4)$ \\
\hline$C(34)$ & $27(5)$ & $37(5)$ & $26(4)$ & $0(4)$ & $2(4)$ & $3(4)$ \\
\hline$C(35)$ & $41(6)$ & $28(5)$ & $32(5)$ & $-3(4)$ & $6(4)$ & $4(4)$ \\
\hline$C(36)$ & $26(5)$ & $32(5)$ & $36(5)$ & $3(4)$ & $-1(4)$ & $9(4)$ \\
\hline $\mathrm{C}(37)$ & $26(5)$ & $27(5)$ & $36(5)$ & $6(4)$ & $2(4)$ & $-6(4)$ \\
\hline C(39) & $46(7)$ & $56(7)$ & $30(5)$ & $-14(5)$ & $1(4)$ & $25(6)$ \\
\hline $\mathrm{C}(38)$ & $38(6)$ & $27(4)$ & $43(5)$ & $12(4)$ & $-7(4)$ & $-8(4)$ \\
\hline $\mathrm{C}(40)$ & $40(6)$ & $40(6)$ & $42(6)$ & $-2(5)$ & $1(5)$ & $12(5)$ \\
\hline $\mathrm{C}(41)$ & $41(5)$ & $21(4)$ & $19(4)$ & $-5(3)$ & $-8(4)$ & $-3(4)$ \\
\hline $\mathrm{C}(42)$ & $30(5)$ & $34(5)$ & $27(4)$ & $-4(4)$ & $-2(4)$ & $3(4)$ \\
\hline $\mathrm{C}(43)$ & $53(6)$ & $29(5)$ & $28(5)$ & $-3(4)$ & $-7(5)$ & $4(5)$ \\
\hline$C(44)$ & $42(6)$ & $28(5)$ & $35(5)$ & $0(4)$ & $-10(4)$ & $6(4)$ \\
\hline $\mathrm{C}(45)$ & $32(5)$ & $34(5)$ & $45(6)$ & $-11(4)$ & $-9(5)$ & $-1(4)$ \\
\hline$C(46)$ & $42(5)$ & $28(4)$ & $25(4)$ & $-11(4)$ & $2(5)$ & $-13(4)$ \\
\hline $\mathrm{C}(47)$ & $80(9)$ & $36(6)$ & $38(5)$ & $-2(5)$ & $-12(6)$ & $10(6)$ \\
\hline $\mathrm{C}(48)$ & $50(6)$ & $37(5)$ & $59(7)$ & $-1(5)$ & $-12(6)$ & $3(5)$ \\
\hline C(49) & $25(5)$ & $36(5)$ & $36(5)$ & $7(4)$ & $-7(4)$ & $4(4)$ \\
\hline $\mathrm{C}(50)$ & $32(5)$ & $36(5)$ & $43(6)$ & $1(4)$ & $-2(4)$ & $-7(4)$ \\
\hline $\mathrm{C}(51)$ & $37(5)$ & $33(5)$ & $37(5)$ & $-6(4)$ & $-5(4)$ & $1(5)$ \\
\hline$C(52)$ & $39(6)$ & $39(5)$ & $34(5)$ & $-2(4)$ & $-14(4)$ & $-2(5)$ \\
\hline $\mathrm{C}(53)$ & $38(5)$ & $41(5)$ & $27(4)$ & $-8(4)$ & $-9(4)$ & $3(5)$ \\
\hline$C(54)$ & $30(5)$ & $39(5)$ & $30(4)$ & $3(4)$ & $-1(4)$ & $-4(5)$ \\
\hline
\end{tabular}




\begin{tabular}{|c|c|c|c|c|c|c|}
\hline$C(55)$ & $36(6)$ & $36(5)$ & $69(8)$ & $-9(5)$ & $-7(5)$ & $-12(5)$ \\
\hline$C(56)$ & $95(11)$ & $39(6)$ & $41(6)$ & $1(5)$ & $5(7)$ & $-30(7)$ \\
\hline$C(57)$ & $72(4)$ & $71(4)$ & $70(4)$ & $0(1)$ & $0(1)$ & $0(1)$ \\
\hline $\mathrm{C}(58)$ & $100(5)$ & $99(5)$ & $99(5)$ & $0(1)$ & $0(1)$ & $0(1)$ \\
\hline C(59) & $76(9)$ & $89(10)$ & $63(8)$ & $19(7)$ & $20(8)$ & $-14(8)$ \\
\hline $\mathrm{C}(60)$ & $105(6)$ & $105(6)$ & $104(6)$ & $1(1)$ & $0(1)$ & $0(1)$ \\
\hline $\mathrm{B}(1)$ & $37(6)$ & $35(5)$ & $25(4)$ & $7(4)$ & $-3(4)$ & $-2(5)$ \\
\hline $\mathrm{F}(1 \mathrm{~A})$ & $73(4)$ & $73(4)$ & $72(4)$ & $-1(1)$ & $-1(1)$ & 1(1) \\
\hline $\mathrm{F}(1 \mathrm{~B})$ & $56(4)$ & $55(3)$ & $54(3)$ & $0(1)$ & $-1(1)$ & $0(1)$ \\
\hline $\mathrm{F}(2)$ & $99(3)$ & $102(3)$ & $99(3)$ & $1(1)$ & $-1(1)$ & $1(1)$ \\
\hline $\mathrm{F}(3)$ & 107(3) & 107(3) & $106(3)$ & $1(1)$ & $-1(1)$ & $-1(1)$ \\
\hline $\mathrm{F}(4)$ & 101(3) & 101(3) & $103(3)$ & $-2(1)$ & $-1(1)$ & $-1(1)$ \\
\hline $\mathrm{F}(5)$ & $87(3)$ & $89(3)$ & $87(3)$ & $1(1)$ & $0(1)$ & $-2(1)$ \\
\hline $\mathrm{F}(6)$ & $72(5)$ & $93(6)$ & $84(6)$ & $25(5)$ & $-40(5)$ & $-34(4)$ \\
\hline $\mathrm{F}(7)$ & 124(7) & $90(5)$ & $43(4)$ & $-12(3)$ & $-32(4)$ & $46(5)$ \\
\hline $\mathrm{F}(8)$ & $73(2)$ & $75(2)$ & $72(2)$ & $-2(1)$ & $0(1)$ & $2(1)$ \\
\hline $\mathrm{F}(9)$ & $60(4)$ & $98(5)$ & $67(4)$ & $-23(4)$ & $-14(4)$ & $-18(5)$ \\
\hline $\mathrm{F}(10)$ & $30(3)$ & $47(4)$ & $101(5)$ & $10(3)$ & $-11(3)$ & $-11(3)$ \\
\hline $\mathrm{F}(11)$ & $47(4)$ & $47(4)$ & 73(4) & $1(3)$ & $-7(3)$ & 11(3) \\
\hline $\mathrm{F}(12)$ & $45(4)$ & $68(4)$ & $63(4)$ & $0(3)$ & $-18(3)$ & $12(3)$ \\
\hline $\mathrm{F}(13)$ & $89(3)$ & $87(3)$ & $86(3)$ & $0(1)$ & $0(1)$ & 1(1) \\
\hline $\mathrm{F}(14)$ & $65(5)$ & $127(7)$ & $76(5)$ & $61(5)$ & $3(4)$ & $-8(5)$ \\
\hline $\mathrm{F}(15)$ & $96(6)$ & $56(4)$ & $51(4)$ & $16(3)$ & $-7(4)$ & $20(4)$ \\
\hline $\mathrm{F}(16)$ & $93(3)$ & $95(3)$ & $94(3)$ & $1(1)$ & $0(1)$ & 1(1) \\
\hline $\mathrm{F}(17)$ & $87(3)$ & $89(3)$ & $87(3)$ & $-2(1)$ & $1(1)$ & $-1(1)$ \\
\hline $\mathrm{F}(18)$ & $96(3)$ & $96(3)$ & $98(3)$ & $0(1)$ & $1(1)$ & $-1(1)$ \\
\hline $\mathrm{F}(19)$ & $84(2)$ & $83(2)$ & $82(2)$ & $-2(1)$ & $1(1)$ & $-2(1)$ \\
\hline $\mathrm{F}(20)$ & $72(5)$ & $85(5)$ & $46(4)$ & $-23(4)$ & $16(4)$ & 11(4) \\
\hline $\mathrm{F}(21)$ & $81(2)$ & $80(2)$ & $77(2)$ & $0(1)$ & $0(1)$ & $2(1)$ \\
\hline $\mathrm{F}(22)$ & $100(3)$ & $100(3)$ & $100(3)$ & $-1(1)$ & $2(1)$ & $-2(1)$ \\
\hline $\mathrm{F}(23)$ & $70(5)$ & $116(7)$ & $75(5)$ & $31(5)$ & $29(4)$ & $-17(5)$ \\
\hline $\mathrm{F}(24)$ & $46(4)$ & $84(5)$ & $153(8)$ & $20(6)$ & $41(5)$ & $11(4)$ \\
\hline $\mathrm{Fe}(1)$ & $36(1)$ & $29(1)$ & $49(1)$ & $3(1)$ & $2(1)$ & $2(1)$ \\
\hline $\mathrm{Fe}(2)$ & $33(1)$ & $32(1)$ & $51(1)$ & $-2(1)$ & $2(1)$ & $1(1)$ \\
\hline $\operatorname{In}(1)$ & $35(1)$ & $31(1)$ & $33(1)$ & $2(1)$ & $2(1)$ & $1(1)$ \\
\hline $\mathrm{O}(1)$ & $84(3)$ & $84(3)$ & $84(3)$ & $0(1)$ & $1(1)$ & $0(1)$ \\
\hline $\mathrm{O}(2)$ & $68(6)$ & $42(5)$ & $111(8)$ & $12(5)$ & $-47(5)$ & $2(4)$ \\
\hline $\mathrm{O}(3)$ & 109(8) & $79(6)$ & $66(6)$ & $27(5)$ & $-29(6)$ & $10(6)$ \\
\hline $\mathrm{O}(4)$ & $69(6)$ & $53(5)$ & $89(7)$ & $-16(5)$ & $46(5)$ & $-6(5)$ \\
\hline $\mathrm{O}(5)$ & $130(9)$ & $77(6)$ & $45(4)$ & $-4(4)$ & $20(5)$ & $10(6)$ \\
\hline
\end{tabular}


Table 4.5 Hydrogen coordinates ( x $10^{4}$ ) and isotropic displacement parameters $\left(\AA^{2} \times 10^{3}\right)$ for 14 .

\begin{tabular}{|c|c|c|c|c|}
\hline & $\mathrm{x}$ & $\mathrm{y}$ & z & $\mathrm{U}(\mathrm{eq})$ \\
\hline $\mathrm{H}(6 \mathrm{~A})$ & 7087 & 3255 & 6440 & 77 \\
\hline $\mathrm{H}(6 \mathrm{~B})$ & 7492 & 4071 & 6691 & 77 \\
\hline $\mathrm{H}(6 \mathrm{C})$ & 7397 & 3970 & 6037 & 77 \\
\hline $\mathrm{H}(7 \mathrm{~A})$ & 5286 & 3940 & 5228 & 120 \\
\hline $\mathrm{H}(7 \mathrm{~B})$ & 5965 & 3297 & 5446 & 120 \\
\hline $\mathrm{H}(7 \mathrm{C})$ & 6251 & 4187 & 5240 & 120 \\
\hline $\mathrm{H}(8 \mathrm{~A})$ & 3886 & 3971 & 5900 & 143 \\
\hline $\mathrm{H}(8 \mathrm{~B})$ & 4220 & 4719 & 5534 & 143 \\
\hline $\mathrm{H}(8 \mathrm{C})$ & 3733 & 4896 & 6097 & 143 \\
\hline $\mathrm{H}(9 \mathrm{~A})$ & 3916 & 5202 & 6976 & 118 \\
\hline $\mathrm{H}(9 \mathrm{~B})$ & 4622 & 5407 & 7421 & 118 \\
\hline $\mathrm{H}(9 \mathrm{C})$ & 4217 & 4510 & 7400 & 118 \\
\hline $\mathrm{H}(10 \mathrm{~A})$ & 5990 & 5033 & 7686 & 102 \\
\hline $\mathrm{H}(10 \mathrm{~B})$ & 6880 & 4823 & 7423 & 102 \\
\hline $\mathrm{H}(10 \mathrm{C})$ & 6299 & 4101 & 7652 & 102 \\
\hline $\mathrm{H}(16 \mathrm{~A})$ & 10709 & 7844 & 6625 & 130 \\
\hline $\mathrm{H}(16 \mathrm{~B})$ & 10481 & 7376 & 6065 & 130 \\
\hline $\mathrm{H}(16 \mathrm{C})$ & 10761 & 6864 & 6597 & 130 \\
\hline $\mathrm{H}(17 \mathrm{~A})$ & 8425 & 7784 & 5572 & 121 \\
\hline $\mathrm{H}(17 \mathrm{~B})$ & 9405 & 7945 & 5624 & 121 \\
\hline $\mathrm{H}(17 \mathrm{C})$ & 8759 & 8608 & 5856 & 121 \\
\hline $\mathrm{H}(18 \mathrm{~A})$ & 7334 & 8419 & 6644 & 97 \\
\hline $\mathrm{H}(18 \mathrm{~B})$ & 6898 & 7565 & 6794 & 97 \\
\hline $\mathrm{H}(18 \mathrm{C})$ & 7193 & 7743 & 6175 & 97 \\
\hline H(19A) & 8020 & 7220 & 7980 & 114 \\
\hline H(19B) & 7985 & 6290 & 7769 & 114 \\
\hline $\mathrm{H}(19 \mathrm{C})$ & 7292 & 6932 & 7573 & 114 \\
\hline $\mathrm{H}(20 \mathrm{~A})$ & 10209 & 6259 & 7470 & 115 \\
\hline $\mathrm{H}(20 \mathrm{~B})$ & 9451 & 6416 & 7886 & 115 \\
\hline $\mathrm{H}(20 \mathrm{C})$ & 10126 & 7122 & 7780 & 115 \\
\hline $\mathrm{H}(26)$ & 7025 & 1433 & 5083 & 39 \\
\hline $\mathrm{H}(28)$ & 8620 & 3299 & 4743 & 41 \\
\hline $\mathrm{H}(30)$ & 8807 & 2071 & 6185 & 47 \\
\hline $\mathrm{H}(34)$ & 7740 & 1599 & 7182 & 36 \\
\hline $\mathrm{H}(36)$ & 5546 & 2692 & 7499 & 38 \\
\hline $\mathrm{H}(38)$ & 5895 & 1459 & 6063 & 43 \\
\hline $\mathrm{H}(42)$ & 7618 & 65 & 7121 & 37 \\
\hline $\mathrm{H}(44)$ & 9967 & -779 & 7202 & 42 \\
\hline $\mathrm{H}(46)$ & 9219 & 603 & 5902 & 38 \\
\hline $\mathrm{H}(50)$ & 6130 & -20 & 6399 & 44 \\
\hline $\mathrm{H}(52)$ & 5969 & -1650 & 5145 & 45 \\
\hline $\mathrm{H}(54)$ & 7787 & 52 & 5159 & 40 \\
\hline $\mathrm{H}(57 \mathrm{~A})$ & 8137 & 4748 & 5027 & 85 \\
\hline $\mathrm{H}(57 \mathrm{~B})$ & 7166 & 4707 & 4887 & 85 \\
\hline $\mathrm{H}(58 \mathrm{~A})$ & 7455 & 4978 & 4101 & 119 \\
\hline $\mathrm{H}(58 \mathrm{~B})$ & 8411 & 5156 & 4263 & 119 \\
\hline $\mathrm{H}(59 \mathrm{~A})$ & 7163 & 6254 & 4109 & 91 \\
\hline $\mathrm{H}(59 \mathrm{~B})$ & 8141 & 6430 & 4157 & 91 \\
\hline $\mathrm{H}(60 \mathrm{~A})$ & 6998 & 6825 & 4934 & 125 \\
\hline $\mathrm{H}(60 \mathrm{~B})$ & 7999 & 6884 & 5019 & 125 \\
\hline
\end{tabular}


5. $\mathrm{X}, \mathrm{y}, \mathrm{z}$ coordinates for DFT optimised geometry of $\left[\left\{\mathrm{Cp}^{*} \mathrm{Fe}(\mathrm{CO})_{2}\right\}(\mu-\mathrm{Ga})\right]^{+}$

\begin{tabular}{|c|c|c|c|}
\hline $\mathrm{Ga}$ & 11.516091000 & 14.045199000 & 16.978772000 \\
\hline $\mathrm{Fe}$ & 12.654783000 & 12.824223000 & 18.615883000 \\
\hline $\mathrm{Fe}$ & 10.424746000 & 15.226920000 & 15.283539000 \\
\hline O & 13.160781000 & 15.372222000 & 20.054601000 \\
\hline O & 15.098738000 & 12.908491000 & 16.943186000 \\
\hline O & 11.346568000 & 17.773024000 & 16.490183000 \\
\hline O & 7.876624000 & 15.080312000 & 16.789093000 \\
\hline $\mathrm{C}$ & 12.946184000 & 14.383233000 & 19.490462000 \\
\hline C & 14.132875000 & 12.908948000 & 17.587529000 \\
\hline C & 10.994875000 & 16.759440000 & 16.053101000 \\
\hline C & 8.889094000 & 15.133232000 & 16.226021000 \\
\hline $\mathrm{C}$ & 11.063608000 & 11.270948000 & 18.561402000 \\
\hline $\mathrm{C}$ & 11.070216000 & 11.942446000 & 19.843470000 \\
\hline C & 12.354192000 & 11.682987000 & 20.474923000 \\
\hline C & 13.147818000 & 10.907078000 & 19.577897000 \\
\hline $\mathrm{C}$ & 12.353575000 & 10.670284000 & 18.368984000 \\
\hline $\mathrm{C}$ & 9.858449000 & 11.057243000 & 17.679605000 \\
\hline C & 9.878784000 & 12.595059000 & 20.499660000 \\
\hline $\mathrm{C}$ & 12.722512000 & 12.066761000 & 21.885615000 \\
\hline C & 14.513083000 & 10.334106000 & 19.861050000 \\
\hline C & 12.766113000 & 9.768817000 & 17.230457000 \\
\hline C & 9.822083000 & 13.883348000 & 13.652023000 \\
\hline C & 11.259411000 & 13.798620000 & 13.801988000 \\
\hline $\mathrm{C}$ & 11.806883000 & 15.113724000 & 13.582142000 \\
\hline C & 10.709368000 & 16.006164000 & 13.244766000 \\
\hline $\mathrm{C}$ & 9.490611000 & 15.265776000 & 13.298687000 \\
\hline $\mathrm{C}$ & 8.851796000 & 12.725712000 & 13.677608000 \\
\hline C & 12.054901000 & 12.521530000 & 13.941491000 \\
\hline C & 13.274031000 & 15.459996000 & 13.511330000 \\
\hline C & 10.859266000 & 17.437519000 & 12.789170000 \\
\hline $\mathrm{C}$ & 8.124859000 & 15.776459000 & 12.920168000 \\
\hline $\mathrm{H}$ & 10.136990000 & 10.909685000 & 16.629724000 \\
\hline $\mathrm{H}$ & 9.144825000 & 11.887316000 & 17.733315000 \\
\hline $\mathrm{H}$ & 9.322949000 & 10.150324000 & 18.001103000 \\
\hline $\mathrm{H}$ & 9.326137000 & 11.855133000 & 21.099154000 \\
\hline $\mathrm{H}$ & 9.179544000 & 13.005045000 & 19.762559000 \\
\hline $\mathrm{H}$ & 10.172189000 & 13.407391000 & 21.172863000 \\
\hline $\mathrm{H}$ & 12.218646000 & 12.985688000 & 22.205738000 \\
\hline $\mathrm{H}$ & 13.802243000 & 12.210281000 & 22.003720000 \\
\hline $\mathrm{H}$ & 12.416937000 & 11.266696000 & 22.577935000 \\
\hline $\mathrm{H}$ & 15.077923000 & 10.959942000 & 20.561128000 \\
\hline $\mathrm{H}$ & 15.110182000 & 10.223320000 & 18.948588000 \\
\hline $\mathrm{H}$ & 14.416904000 & 9.334886000 & 20.314312000 \\
\hline $\mathrm{H}$ & 12.593942000 & 8.715832000 & 17.502806000 \\
\hline $\mathrm{H}$ & 13.830919000 & 9.871804000 & 16.989842000 \\
\hline $\mathrm{H}$ & 12.192234000 & 9.969733000 & 16.318812000 \\
\hline $\mathrm{H}$ & 7.858190000 & 13.031417000 & 14.025718000 \\
\hline $\mathrm{H}$ & 9.202395000 & 11.912956000 & 14.324435000 \\
\hline $\mathrm{H}$ & 8.731381000 & 12.311794000 & 12.664325000 \\
\hline $\mathrm{H}$ & 11.519570000 & 11.763840000 & 14.525281000 \\
\hline $\mathrm{H}$ & 13.032587000 & 12.688320000 & 14.407310000 \\
\hline $\mathrm{H}$ & 12.240107000 & 12.090098000 & 12.945710000 \\
\hline $\mathrm{H}$ & 13.638479000 & 15.346111000 & 12.478408000 \\
\hline $\mathrm{H}$ & 13.881881000 & 14.805222000 & 14.145852000 \\
\hline $\mathrm{H}$ & 13.467436000 & 16.495261000 & 13.81288900 \\
\hline $\mathrm{H}$ & 11.002506000 & 17.462974000 & 11.697451000 \\
\hline $\mathrm{H}$ & 11.727502000 & 17.926710000 & 13.244452000 \\
\hline $\mathrm{H}$ & 9.970815000 & 18.036029000 & 13.01855500 \\
\hline $\mathrm{H}$ & 8.027273000 & 16.851350000 & 13.10873100 \\
\hline
\end{tabular}


13.468928000

7. 944061000

15.610692000

11.846384000 


\section{6. $X, y, z$ coordinates for DFT optimised geometry of $\left[\left\{\mathrm{Cp}^{*} \mathrm{Fe}(\mathrm{CO})_{2}\right\}(\mu-\mathrm{In})\right]^{+}$}

(a) Minimum energy conformer $\left(\vartheta=161.8^{\circ}\right)$

\begin{tabular}{|c|c|c|c|}
\hline $\mathrm{Fe}$ & -2.4173 & -0.0684 & -0.4736 \\
\hline In & 0.0001 & -0.0818 & -0.0011 \\
\hline $\mathrm{Fe}$ & 2.4170 & -0.0695 & 0.4736 \\
\hline C & -2.7338 & 0.1998 & 1.6871 \\
\hline C & -3.2481 & 1.3376 & 0.9497 \\
\hline $\mathrm{C}$ & -4.3037 & 0.8621 & 0.0609 \\
\hline C & -4.4065 & -0.5645 & 0.2242 \\
\hline C & -3.4189 & -0.9785 & 1.2215 \\
\hline C & -5.1983 & 1.7246 & -0.7879 \\
\hline C & 3.2476 & 1.3394 & -0.9469 \\
\hline C & 2.7345 & 0.2027 & -1.6864 \\
\hline C & 3.4202 & -0.9759 & -1.2226 \\
\hline C & 4.4070 & -0.5629 & -0.2242 \\
\hline C & 4.3031 & 0.8631 & -0.0586 \\
\hline C & 3.2819 & -2.3581 & -1.8052 \\
\hline C & 5.4267 & -1.4618 & 0.4210 \\
\hline C & -5.4259 & -1.4630 & -0.4221 \\
\hline C & 1.8206 & 0.2659 & -2.8876 \\
\hline C & 5.1968 & 1.7248 & 0.7920 \\
\hline C & -2.9057 & 2.7830 & 1.2013 \\
\hline C & -3.2793 & -2.3616 & 1.8017 \\
\hline $\mathrm{C}$ & 2.9044 & 2.7851 & -1.1962 \\
\hline C & -1.8194 & 0.2617 & 2.8880 \\
\hline C & -2.2133 & -1.5390 & -1.4189 \\
\hline O & -2.1296 & -2.5343 & -2.0504 \\
\hline C & -2.0924 & 0.9841 & -1.8436 \\
\hline 0 & -1.9205 & 1.7062 & -2.7645 \\
\hline $\mathrm{C}$ & 2.2127 & -1.5423 & 1.4154 \\
\hline O & 2.1288 & -2.5391 & 2.0446 \\
\hline C & 2.0916 & 0.9797 & 1.8460 \\
\hline 0 & 1.9193 & 1.6996 & 2.7686 \\
\hline $\mathrm{H}$ & -4.6925 & 2.6318 & -1.1336 \\
\hline $\mathrm{H}$ & -5.5677 & 1.1895 & -1.6682 \\
\hline $\mathrm{H}$ & -6.0747 & 2.0416 & -0.2047 \\
\hline $\mathrm{H}$ & -6.3223 & -1.5314 & 0.2108 \\
\hline $\mathrm{H}$ & -5.7435 & -1.0843 & -1.3986 \\
\hline $\mathrm{H}$ & -5.0494 & -2.4812 & -0.5637 \\
\hline $\mathrm{H}$ & -4.0158 & -2.5100 & 2.6045 \\
\hline $\mathrm{H}$ & -3.4539 & -3.1420 & 1.0538 \\
\hline $\mathrm{H}$ & -2.2884 & -2.5309 & 2.2361 \\
\hline $\mathrm{H}$ & -3.6005 & 3.2150 & 1.9358 \\
\hline $\mathrm{H}$ & -1.8942 & 2.9042 & 1.6040 \\
\hline $\mathrm{H}$ & -2.9789 & 3.3879 & 0.2915 \\
\hline $\mathrm{H}$ & -2.4226 & 0.3921 & 3.7976 \\
\hline $\mathrm{H}$ & -1.2419 & -0.6597 & 3.0281 \\
\hline $\mathrm{H}$ & -1.1248 & 1.1091 & 2.8526 \\
\hline $\mathrm{H}$ & 1.1258 & 1.1131 & -2.8514 \\
\hline $\mathrm{H}$ & 2.4242 & 0.3977 & -3.7968 \\
\hline $\mathrm{H}$ & 1.2433 & -0.6555 & -3.0292 \\
\hline $\mathrm{H}$ & 2.2913 & -2.5273 & -2.2406 \\
\hline $\mathrm{H}$ & 4.0189 & -2.5046 & -2.6079 \\
\hline $\mathrm{H}$ & 3.4566 & -3.1397 & -1.0586 \\
\hline $\mathrm{H}$ & 5.7439 & -1.0844 & 1.3982 \\
\hline $\mathrm{H}$ & 5.0507 & -2.4804 & 0.5611 \\
\hline $\mathrm{H}$ & 6.3233 & -1.5288 & -0.2118 \\
\hline $\mathrm{H}$ & 4.6903 & 2.6313 & 1.1387 \\
\hline
\end{tabular}




$\begin{array}{lllr}\mathrm{H} & 5.5659 & 1.1886 & 1.6718 \\ \mathrm{H} & 6.0734 & 2.0430 & 0.2098 \\ \mathrm{H} & 3.5991 & 3.2188 & -1.9298 \\ \mathrm{H} & 1.8930 & 2.9063 & -1.5991 \\ \mathrm{H} & 2.9769 & 3.3885 & -0.2854\end{array}$

(b) Conformer approximating to solid state structure $\left(\vartheta=82.4^{\circ}\right)$

\begin{tabular}{|c|c|c|c|}
\hline $\mathrm{Fe}$ & 2.4491 & -0.3858 & 0.2697 \\
\hline In & -0.0003 & -0.3484 & -0.0403 \\
\hline $\mathrm{Fe}$ & -2.4488 & -0.3078 & -0.3550 \\
\hline C & 2.6184 & 1.3015 & -1.1236 \\
\hline C & 2.9794 & 1.7150 & 0.2203 \\
\hline $\mathrm{C}$ & 4.1375 & 0.9348 & 0.6336 \\
\hline $\mathrm{C}$ & 4.4593 & 0.0136 & -0.4259 \\
\hline C & 3.5136 & 0.2369 & -1.5165 \\
\hline $\mathrm{C}$ & 4.9202 & 1.1309 & 1.9041 \\
\hline C & -3.0398 & 1.7175 & 0.1357 \\
\hline C & -2.6061 & 1.0628 & 1.3553 \\
\hline $\mathrm{C}$ & -3.4450 & -0.0965 & 1.5610 \\
\hline $\mathrm{C}$ & -4.4361 & -0.1342 & 0.4887 \\
\hline C & -4.1917 & 0.9880 & -0.3799 \\
\hline C & -3.4223 & -1.0053 & 2.7619 \\
\hline C & -5.5821 & -1.1034 & 0.3837 \\
\hline C & 5.6344 & -0.9257 & -0.4620 \\
\hline C & -1.6150 & 1.6120 & 2.3529 \\
\hline $\mathrm{C}$ & -5.0368 & 1.3995 & -1.5554 \\
\hline C & 2.4175 & 2.8952 & 0.9689 \\
\hline $\mathrm{C}$ & 3.5766 & -0.4088 & -2.8756 \\
\hline C & -2.5419 & 3.0378 & -0.3921 \\
\hline C & 1.6451 & 2.0092 & -2.0356 \\
\hline $\mathrm{C}$ & 2.4391 & -2.0871 & -0.1894 \\
\hline O & 2.5022 & -3.2212 & -0.5106 \\
\hline $\mathrm{C}$ & 2.1870 & -0.7002 & 1.9807 \\
\hline O & 2.0602 & -0.9032 & 3.1384 \\
\hline $\mathrm{C}$ & -2.4359 & -2.0699 & -0.3215 \\
\hline O & -2.4981 & -3.2481 & -0.2840 \\
\hline $\mathrm{C}$ & -2.1836 & -0.1924 & -2.0903 \\
\hline O & -2.0562 & -0.1042 & -3.2623 \\
\hline $\mathrm{H}$ & 4.2923 & 1.4837 & 2.7287 \\
\hline $\mathrm{H}$ & 5.4128 & 0.2088 & 2.2279 \\
\hline $\mathrm{H}$ & 5.7056 & 1.8836 & 1.7457 \\
\hline $\mathrm{H}$ & 6.5099 & -0.4211 & -0.8955 \\
\hline $\mathrm{H}$ & 5.9157 & -1.2664 & 0.5395 \\
\hline $\mathrm{H}$ & 5.4346 & -1.8124 & -1.0724 \\
\hline $\mathrm{H}$ & 4.2896 & 0.1318 & -3.5145 \\
\hline $\mathrm{H}$ & 3.9129 & -1.4493 & -2.8241 \\
\hline $\mathrm{H}$ & 2.6078 & -0.3947 & -3.3858 \\
\hline $\mathrm{H}$ & 3.0202 & 3.7932 & 0.7704 \\
\hline $\mathrm{H}$ & 1.3901 & 3.1251 & 0.6650 \\
\hline $\mathrm{H}$ & 2.4215 & 2.7362 & 2.0523 \\
\hline $\mathrm{H}$ & 2.1699 & 2.8018 & -2.5878 \\
\hline $\mathrm{H}$ & 1.2066 & 1.3406 & -2.7845 \\
\hline $\mathrm{H}$ & 0.8297 & 2.4963 & -1.4872 \\
\hline $\mathrm{H}$ & -0.8336 & 2.2200 & 1.8819 \\
\hline $\mathrm{H}$ & -2.1369 & 2.2671 & 3.0650 \\
\hline $\mathrm{H}$ & -1.1330 & 0.8252 & 2.9442 \\
\hline $\mathrm{H}$ & -2.4263 & -1.0714 & 3.2125 \\
\hline $\mathrm{H}$ & -4.1060 & -0.6260 & 3.5349 \\
\hline
\end{tabular}




$\begin{array}{lrrr}\mathrm{H} & -3.7452 & -2.0219 & 2.5152 \\ \mathrm{H} & -5.9063 & -1.2405 & -0.6527 \\ \mathrm{H} & -5.3288 & -2.0891 & 0.7874 \\ \mathrm{H} & -6.4461 & -0.7299 & 0.9518 \\ \mathrm{H} & -4.4566 & 1.9296 & -2.3177 \\ \mathrm{H} & -5.5172 & 0.5414 & -2.0356 \\ \mathrm{H} & -5.8354 & 2.0778 & -1.2223 \\ \mathrm{H} & -3.1651 & 3.8599 & -0.0118 \\ \mathrm{H} & -1.5122 & 3.2451 & -0.0795 \\ \mathrm{H} & -2.5774 & 3.0831 & -1.4857\end{array}$

(c) Rotational profile

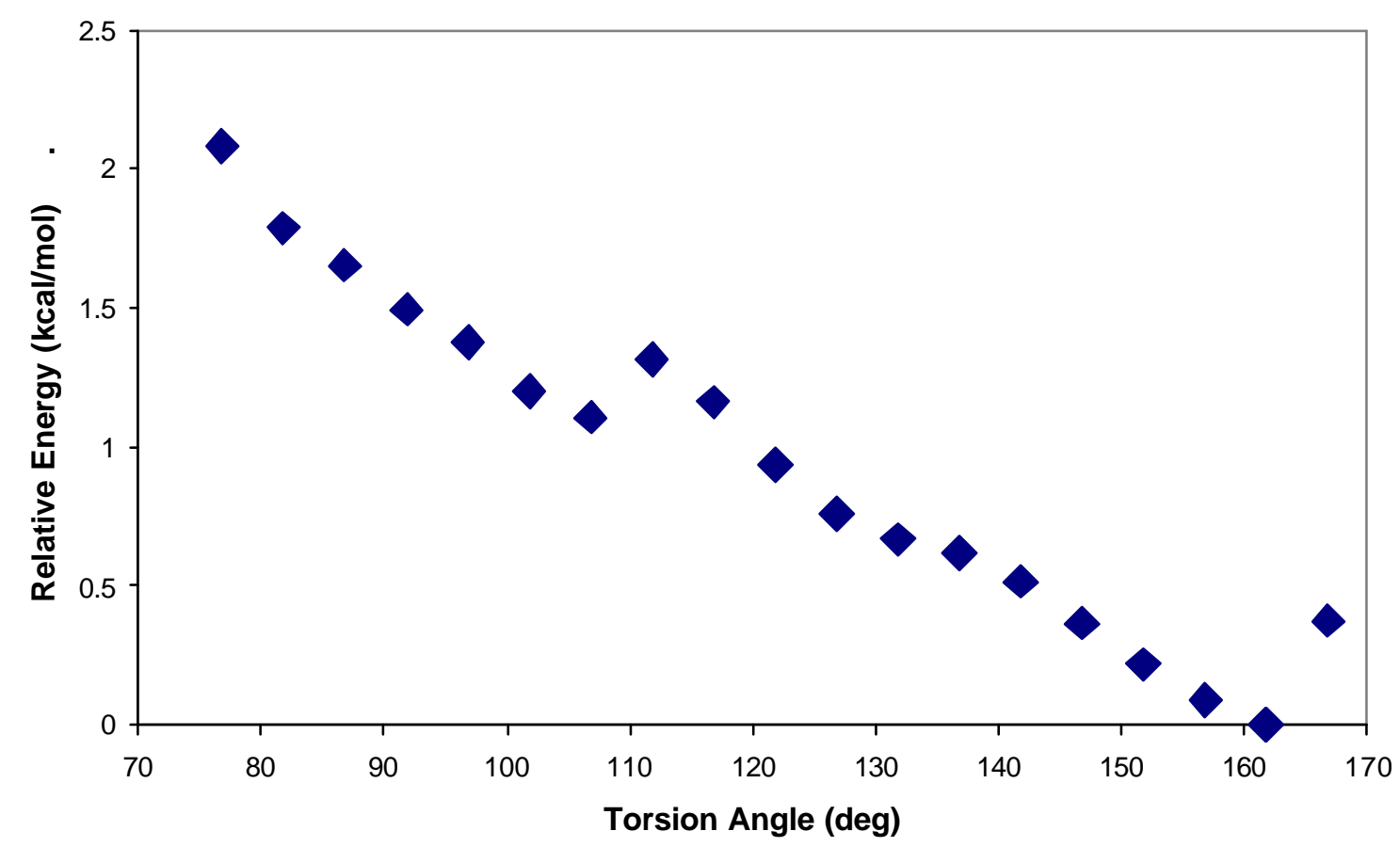


7. ${ }^{1}$ H NMR spectra for new compounds

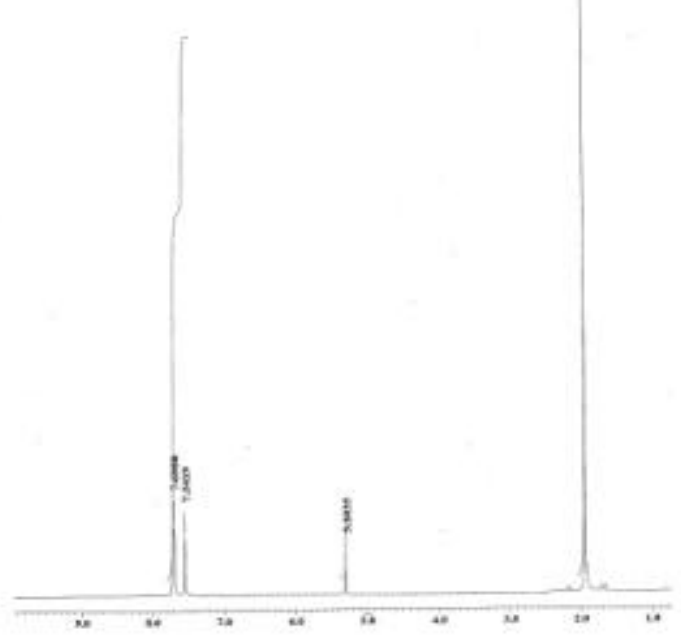

$\left.\left[\left\{\mathrm{Cp} * \mathrm{Fe}(\mathrm{CO})_{2}\right\}_{2}(\mu-\mathrm{Ga})\right\}\right]^{+}\left[\mathrm{BAr}_{4}^{f}\right]^{-}, \mathbf{7}\left(\mathrm{CD}_{2} \mathrm{Cl}_{2}\right)$

64 


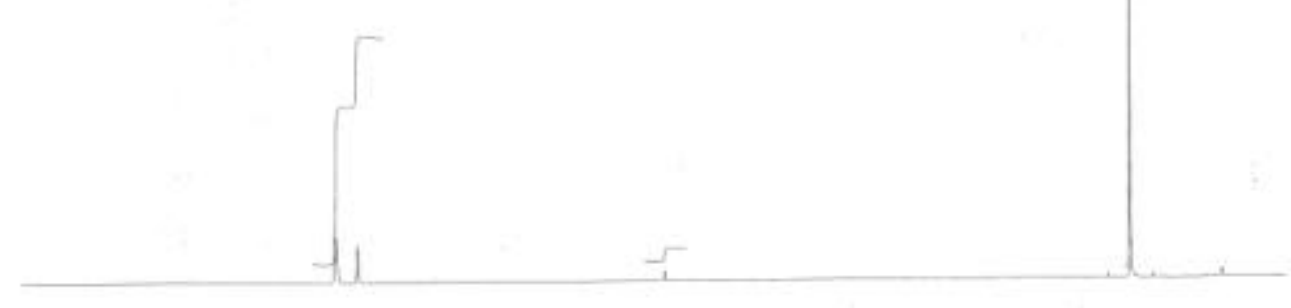

$\left.\left[\left\{\mathrm{Cp} * \mathrm{Fe}(\mathrm{CO})_{2}\right\}_{2}(\mu-\mathrm{In})\right\}\right]^{+}\left[\mathrm{BAr}_{4}^{f}\right]^{-}, \mathbf{8}\left(\mathrm{CD}_{2} \mathrm{Cl}_{2}\right)$ 


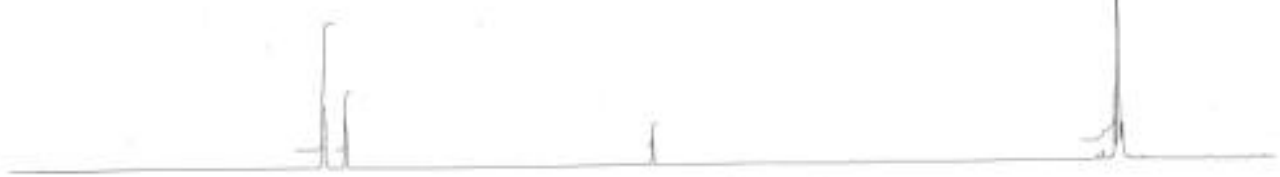

$\left.\left[\left\{\mathrm{Cp} * \mathrm{Fe}(\mathrm{CO})_{2}\right\}_{2}(\mu-\mathrm{I})\right\}\right]^{+}\left[\mathrm{BAr}_{4}^{f}\right]^{-}, \mathbf{9}\left(\mathrm{CD}_{2} \mathrm{Cl}_{2}\right)$ 


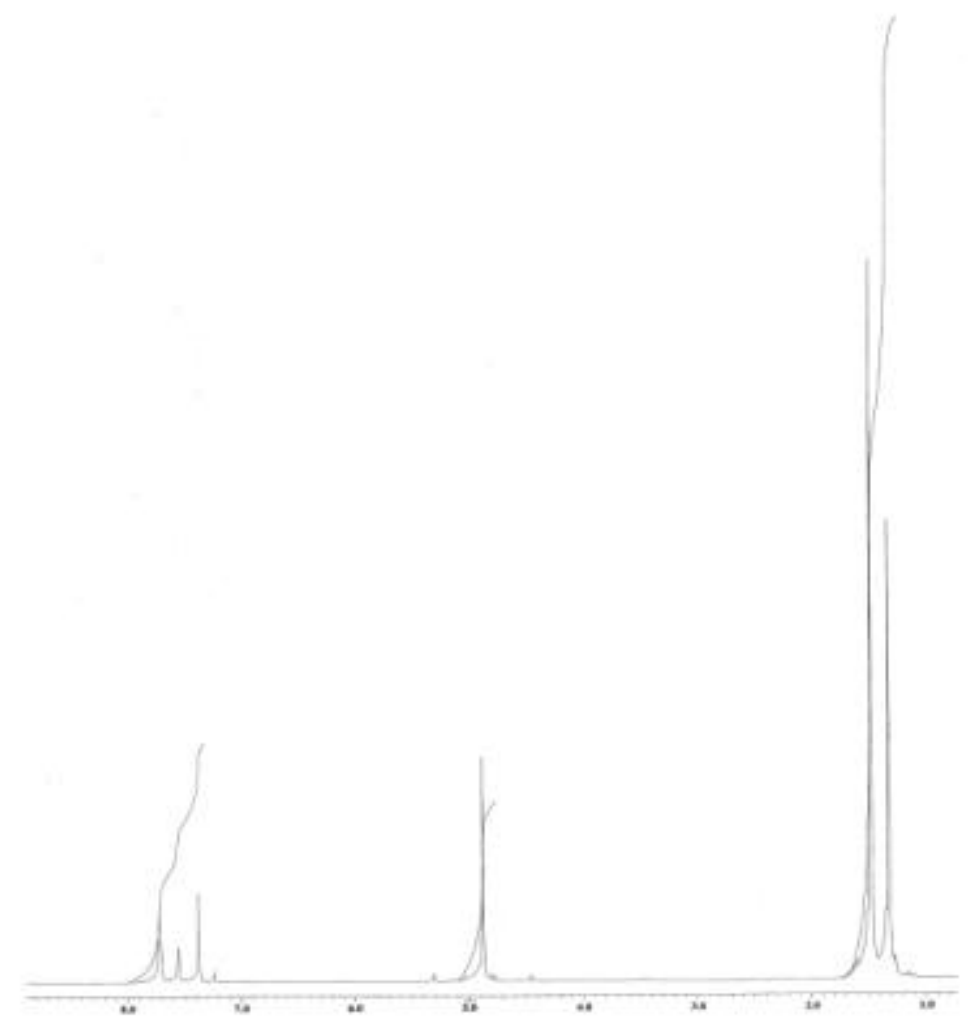

$\left[\left\{\mathrm{CpFe}(\mathrm{CO})_{2} \mathrm{Ga}\left(\mathrm{Mes}^{*}\right)\right\}_{2}(\mu-\mathrm{Cl})\right]^{+}\left[\mathrm{BAr}_{4}^{f}\right]^{-}, \mathbf{1 0}\left(\mathrm{CD}_{2} \mathrm{Cl}_{2}\right)$ 


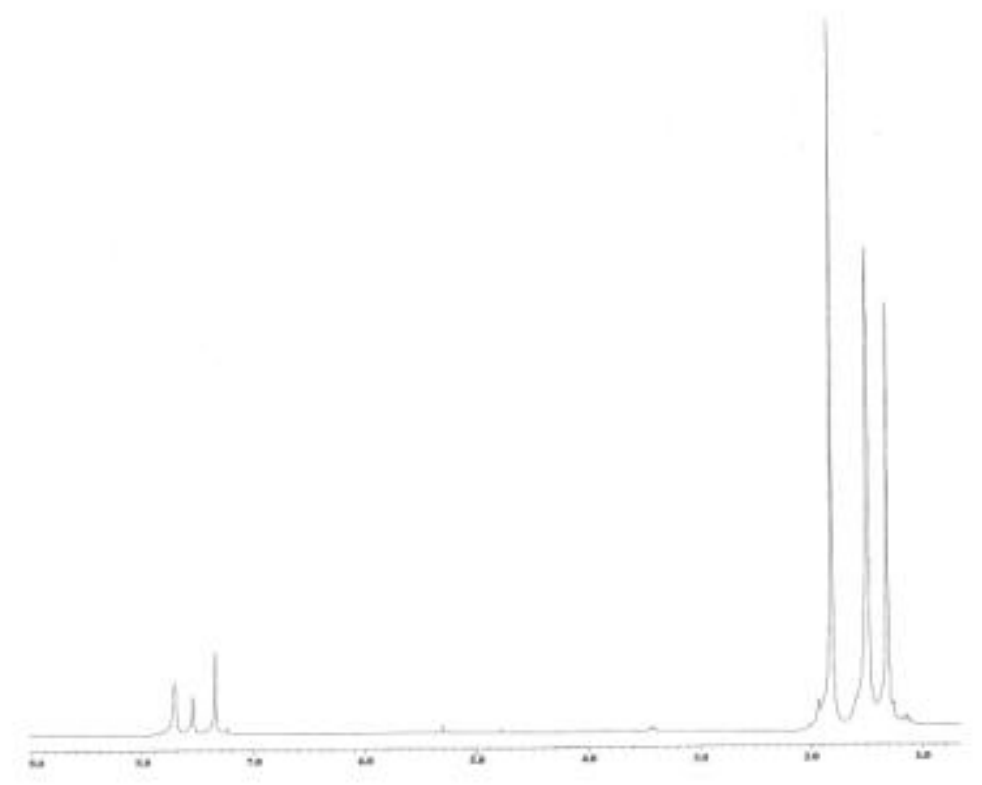

$\left[\left\{\mathrm{Cp} * \mathrm{Fe}(\mathrm{CO})_{2} \mathrm{Ga}(\mathrm{Mes} *)\right\}_{2}(\mu-\mathrm{Cl})\right]^{+}\left[\mathrm{BAr}_{4}^{f}\right]^{-}, \mathbf{1 1}\left(\mathrm{CD}_{2} \mathrm{Cl}_{2}\right)$ 


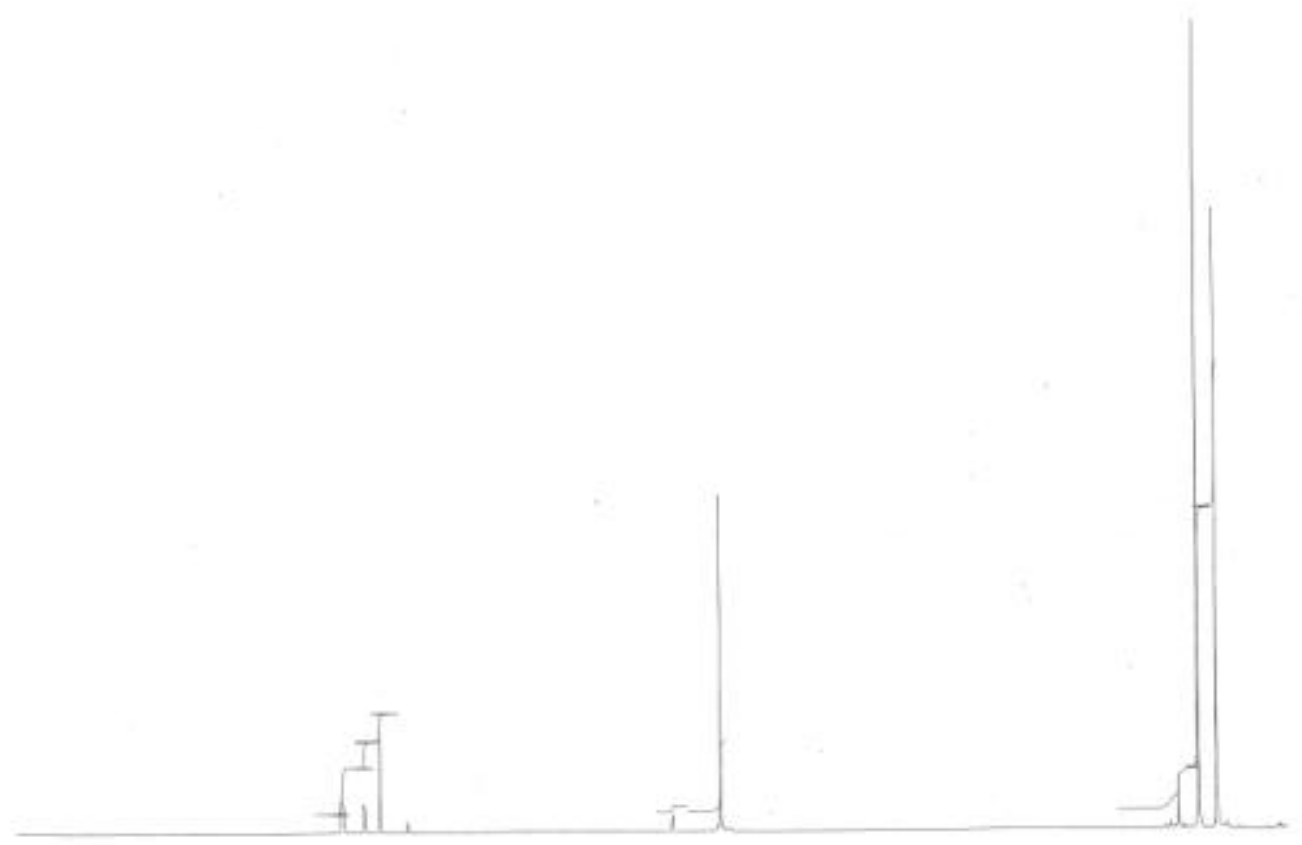

$\left[\left\{\mathrm{CpFe}(\mathrm{CO})_{2} \mathrm{In}\left(\mathrm{Mes}^{*}\right)\right\}_{2}(\mu-\mathrm{Br})\right]^{+}\left[\mathrm{BAr}_{4}^{f}\right]^{-}, \mathbf{1 2}\left(\mathrm{CD}_{2} \mathrm{Cl}_{2}\right)$ 


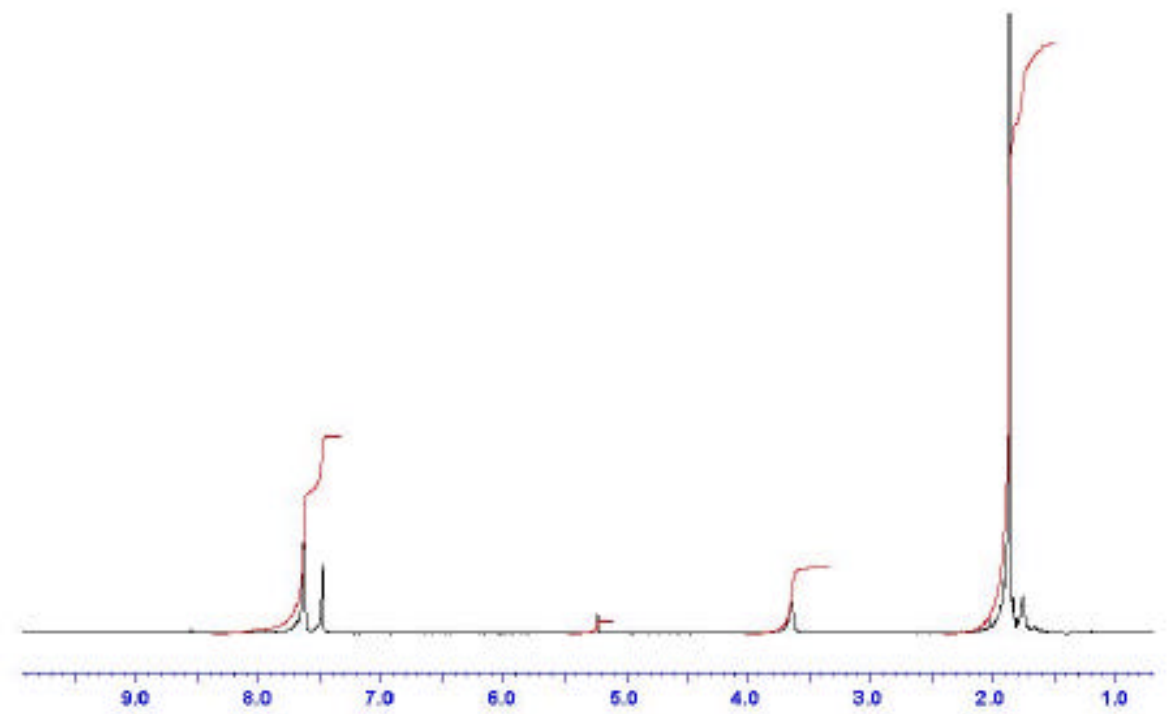

$\left[\left\{\mathrm{Cp} * \mathrm{Fe}(\mathrm{CO})_{2}\right\}_{2}\{\mu-\mathrm{Ga}(\mathrm{thf})\}\right]^{+}\left[\mathrm{BAr}_{4}^{f}\right]^{-}, \mathbf{1 3}\left(\mathrm{CD}_{2} \mathrm{Cl}_{2}\right)$ 


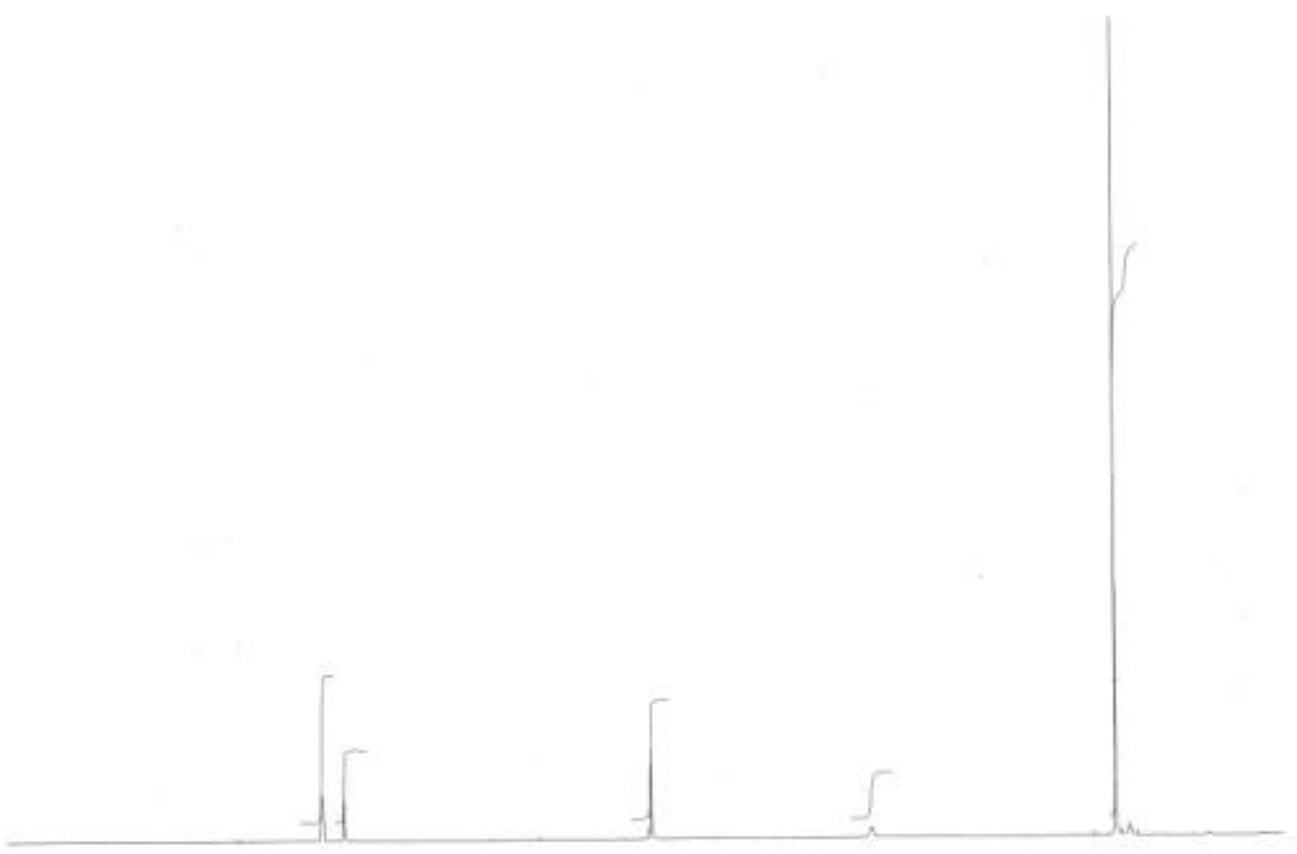

$\left[\left\{\mathrm{Cp} * \mathrm{Fe}(\mathrm{CO})_{2}\right\}_{2}\{\mu-\operatorname{In}(\text { thf })\}\right]^{+}\left[\mathrm{BAr}_{4}^{f}\right]^{-}, \mathbf{1 4}\left(\mathrm{CD}_{2} \mathrm{Cl}_{2}\right)$ 